\title{
LOOPS DE MOUFANG LINEARES
}

\author{
Maria de Lourdes Merlini Giuliani
}

TESE APRESENTADA

$\mathrm{AO}$

INSTITUTO DE MATEMÁTICA E ESTATÍSTICA

DA

UNIVERSIDADE DE SÃO PAULO

PARA

OBTENÇÃO DO GRAU

DE

DOUTOR EM MATEMÁTICA

\author{
Área de Concentração: Álgebra \\ Orientador: Prof. Dr. Francisco César Polcino Milies
}

- São Paulo, março de 1998 -

Este trabalho teve apoio financeiro da CAPES. 


\section{LOOPS DE MOUFANG LINEARES}

Este exemplar corresponde à redação final da tese apresentada por Maria de Lourdes Merlini Giuliani, devidamente corrigida e aprovada pela Comissão Julgadora

São Paulo, 13 de março de 1998.

\section{Comissão Julgadora}

- Prof. Dr. Francisco César Polcino Milies (Presidente) - IME-USP.

- Prof. Dr. Luiz Antonio Peresi - IME-USP.

- Prof. Dr. Antonio Paques - IMECC-UNICAMP.

- Prof. Dr. Guilherme Augusto de La Rocque Leal - URFJ.

- Prof. Dr. Norai Romeu Rocco - UnB. 


\section{Resumo}

Neste trabalho se consideram as álgebras matriciais-vetoriais de Zorn que são, precisamente, as álgebras alternativas simples de dimensão finita. O conjunto dos elementos inversíveis de uma tal álgebra forma um Loop de Moufang. Por analogia com o caso associativo, estes loops serão chamados de loops lineares e denotados na forma $G L L(K)$; subloop formado pelas matrizes de determinante 1 é denotado por $S L L(K)$ e o quociente deste loop pelo seu centro, por $P S L L(K)$.

Inicialmente, demonstramos que as álgebras de Zorn são irredutíveis, no sentido de que não contêm nenhum ideal lateral próprio. Depois, determinamos um conjunto de geradores para $G L L\left(K^{\prime}\right)$ e, conseqüentemente, também para $S L L(K)$. Esta descrição se utiliza para dar uma nova prova de que os loops do tipo $P S L L\left(K^{\circ}\right)$ são simples. Também calculamos as ordens de todos estes loops no caso em que o corpo $K$ é finito.

No último capítulo, estudamos detalhadamente o reticulado de subloops de $G L L\left(F_{2}\right)$. 


\section{Abstract}

In this work we consider Zorn's vector-matrix algebras which are, precisely, the finite dimensional, simple, alternative algebras. The set of invertible elements of such an algebra is a Moufang Loop. As in the associative case, they will be called linear loops and will be denoted by $G L L(K)$; the subloop of all matrices of determinant 1 will be denoted by $S L L(K)$ and the quotient of this loop by its centre by $P S L L\left(K^{\prime}\right)$.

Initially, we show that Zorn's algebras are irreducible, in the sense that they contain no proper one-sided ideal. Then we determine a set of gerators for $G L L(K)$ and, consequently, also for $S L L(K)$. This description is used to give a new proof that loops of type $P S L L(K)$ are simple. We also compute the orders of all these loops in the case when $K$ is finite.

In the last chapter, we study the lattice of subloops of $G L L\left(F_{2}\right)$. 


\section{Agradecimentos}

Agradeço ao Prof. César, orientador e amigo, pela forma competente e dedicada como conduziu a elaboração desta tese.

Agradeço a todos os colegas que direta ou indiretamente me ajudaram durante o período de doutorado. Em especial ao Thierry, pela disponibilidade e paciência de rever muitos detalhes deste trabalho.

Agradeço ao Osmar, pelo companheirismo e incentivo nos momentos difíceis. 
Aos meus filhos, Caio e Emília. 


\section{Conteúdo}

Introdução $\quad 1$

1 Resultados Preliminares 2

1.1 Álgebras Alternativas . . . . . . . . . . . . . . . . . . . 2

1.2 Álgebra Matricial-Vetorial de Zorn . . . . . . . . . . . . . . . 6

1.3 Loops . . . . . . . . . . . . . . . . . . . 7

2 O Loop das Unidades da Álgebra de Zorn 14

2.1 A Álgebra de Zorn . . . . . . . . . . . . . . . . . . . . 14

2.2 O Loop Linear Geral . . . . . . . . . . . . . . . . . . 17

2.3 A Simplicidade de $\operatorname{PSLL}(K) \ldots \ldots \ldots \ldots \ldots$

2.4 As Ordens dos Loops Lineares Finitos . . . . . . . . . . . . . . . 37

3 O Menor Loop Linear $\quad 43$

3.1 Elementos de $G L L\left(F_{2}\right)$ - resultados preliminares . . . . . . . . . . 43

3.2 A Simplicidade de $P S L L\left(F_{2}\right) \ldots \ldots \ldots \ldots$. . . . . . . . . . . 46

3.3 Limitando os Subloops de Ordem Potência de $2 \ldots \ldots . . . . . . . .48$

3.4 Classificação dos 2-subloops de $G L L\left(F_{2}\right) \ldots \ldots \ldots \ldots \ldots$

3.5 A Classificação dos Subloops de $G L L\left(F_{2}\right) \ldots \ldots \ldots$. . . . . . . . 59

$\begin{array}{ll}\text { Referências bibliográficas } & \mathbf{8 1}\end{array}$ 


\section{Introdução}

Em 1956, L. Paige provou que os loops de Moufang, que adiante denotamos por PSLL(K), são simples e, em 1987, M.W. Liebeck provou que todo loop de Moufang não associativo, simples, é isomorfo a um loop da forma $P S L L\left(K^{\prime}\right)$ para algum $K$.

Estes loops são quocientes de loops de unidades de álgebras matriciais-vetoriais de Zorn. que são as álgebras alternativas simples de dimensão finita (não associativas) sobre $K$. Por causa disso, é natural chamar estes loops de loops lineares e denotá-los por $\operatorname{GLL}\left(K^{\circ}\right)$.

Nesta tese, provamos inicialmente que a álgebra de Zorn é mais do que uma álgebra simples: ela é irredutível. Depois, determinamos um conjunto de geradores de $G L L(K)$ e, como conseqüência, obtemos um conjunto de geradores para $\operatorname{PSLL}(K)$ bem menor que o utilizado por Paige e damos uma nova demonstração da simplicidade destes loops. Também provamos que o comutador-associador de $G L L\left(K^{\circ}\right)$ é $S L L\left(K^{\prime}\right)$, o subloop das matrizes de determinante 1. Concluímos o estudo geral dos loops lineares dando uma fórmula explícita para as ordens de $G L L\left(K^{\prime}\right), S L L\left(K^{\prime}\right)$ e $P S L L\left(K^{\prime}\right)$ no caso em que $K^{\prime}$ é um corpo finito.

Finalmente, no Capítulo 3, estudamos em detalhes $\operatorname{PS} L L\left(F_{2}\right)$ quando $F_{2}$ é o corpo com dois elementos e determinamos o reticulado dos seus subloops. 


\section{Capítulo 1}

\section{Resultados Preliminares}

\section{1 Álgebras Alternativas}

Nesta seção, resumimos brevemente alguns resultados sobre álgebras alternativas que serão usadas no próximo capítulo. Quando necessário, damos referências para as demonstrações correspondentes.

Seja $A$ uma álgebra (não associativa) sobre um corpo $K$. Dados $x, y, z \in A$, denotamos o associador de $x, y$ e $z$ e o comutador de $x$ e $y$, respectivamente, por:

$$
\begin{aligned}
(x, y, z) & =(x y) z-x(y z) \\
{[x, y] } & =x y-y x
\end{aligned}
$$

Definição 1.1.1 Dizemos que $A$ é uma álgebra alternativa se para todo par de elementos $x, y \in A$ tem-se que

$$
\begin{array}{ll}
(x, y, y)=0 & \text { (lei alternativa à direita) } \\
(x, x, y)=0 & \text { (lei alternativa à esquerda) }
\end{array}
$$

Essas identidades implicam a lei flexível:

$$
(x, y, x)=0 \quad \forall x, y \in A .
$$


Lema 1.1.2 ([16, p.35]) Em qualquer álgebra alternativa A valem as seguintes identidades:

$$
\begin{aligned}
& x(y z y)=[(x y) z] y, \text { identidade de Moufang à direita; } \\
& (y z y) x=y[z(y x)], \text { identidade de Moufang à esquerda; } \\
& (x y)(z y)=x(y z) x, \text { identidade de Moufang central. }
\end{aligned}
$$

Observação 1.1.3 As identidade (1), (2) e (3) são chamadas identidades de Moufang.

Um resultado muito importante é o seguinte:

Teorema 1.1.4 (Teorema de Artin) ([12, p.29]) Seja A uma álgebra alternativa sobre um corpo $K$. Toda subálgebra $B$ de A, gerada por dois elementos, é associativa.

Definição 1.1.5 Uma álgebra $A$ é de potência associativa se cada elemento de $A$ gera uma subálgebra associativa.

Corolário 1.1.6 ([16, p.37)] Toda álgebra alternativa é de potência associativa.

Definição 1.1.7 Uma álgebra $A$ diz-se simples se seus únicos ideais são $(0)$ e $A$.

Definição 1.1.8 Uma involução numa álgebra $A$ é uma aplicação linear $a \rightarrow \bar{a}$ satisfazendo

$$
\overline{a b}=\bar{b} \bar{a} \quad \text { e } \quad \overline{\bar{a}}=a, \forall a, b \in A \text {. }
$$

Concentraremos nossos resultados numa classe de álgebras alternativas conhecidas como álgebras de Cayley-Dickson. Descreveremos resumidamenteo processo de construção desta álgebra. que pode ser encontrado em [0خ] ou [16]. 


\section{Processo de Cayley-Dickson}

Seja $K$ um corpo de característica $\neq 2$ e $A$ uma álgebra não associativa de dimensão $n$ sobre $K$, com elemento identidade. Suponha que $A$ tem involução $a \rightarrow \bar{a}$ tal que $a+\bar{a} \in K 1$ e $a \bar{a}=\bar{a} a \in K 1$ para todo $a \in A$.

Dado $\alpha \in K$ não nulo e uma indeterminada $\ell$, construímos a álgebra $B$, denotada $(A, \alpha)$ de dimensão $2 n$ sobre $K$ com propriedades similares a $A$.

Os elementos de $B$ são expressões da forma

$$
a+b \ell, \quad a, b \in A \text {. }
$$

A adição e a multiplicação são dadas por

$$
\begin{aligned}
(a+b \ell)+(c+d \ell) & =(a+c)+(b+d) \ell \\
(a+b \ell)(c+d \ell) & =(a c+\alpha \bar{d} b)+(d a+b \bar{c}) \ell
\end{aligned}
$$

Definimos $\overline{a+b \ell}=\bar{a}-b \ell$.

É fácil mostrar que a aplicação $x \rightarrow \bar{x}$ define uma involução em $B$ tal que $x+\bar{x}$ e $x \bar{x}$ estão em $K 1$ para qualquer $x \in B$.

Dados $x=a+b \ell$ e $y=c+d \ell$, pode-se ver que $(x, x, y)=0$ se, e somente se, $A$ é uma álgebra associativa. Assim, temos o seguinte resultado:

Proposição 1.1 .9 ([07, p.17]) Se A é uma álgebra com 1 sobre o corpo $K$ e $\alpha \in K$ não nulo, então $(A, \alpha)$ é alternativa se, e somente se, $A$ é associativa.

Repetindo o processo de construção de $(A, \alpha)$, podemos produzir álgebras de dimensão $4 n, 8 n$, mas poucas destas álgebras são alternativas.

Seja $A_{0}=\Lambda$ e para $a \in A_{0}$, defina $\bar{a}=a$. A aplicação $a \rightarrow \bar{a}$ é certamente uma involução e assim, se $\alpha \in K$ não nulo, podemos construir pelo processo de Cayley-Dickson a 
álgebra bidimensional $A_{1}=\left(A_{0}, \alpha\right)$. Para $0 \neq \beta \in K$, a álgebra $A_{2}=\left(A_{1}, \beta\right)$, de dimensão 4, que denotaremos por $(K, \alpha, \beta)$, é chamada álgebra dos quatérnios generalizada.

Para $\uparrow \in K$ não nulo, a álgebra $A_{3}=\left(A_{2}, \gamma\right)=(K, \alpha, \beta, \gamma)$ de dimensão $\&$ sobre $K$ é chamada álgebra de Cayley-Dickson.

Note que $A_{0}$ é associativa e pode-se verificar que $A_{1}$ e $A_{2}$ também são associativas enquanto que $A_{3}$ é alternativa.

Observemos que uma álgebra $A$ de dimensão 4 sobre $K$ é a álgebra dos quatérnios generalizada se, e somente se, ela tem uma base $\{1, i, j, i j\}$ satisfazendo $i^{2}=\alpha \in K$, $j^{2}=\beta \in K, \alpha \beta \neq 0$ e $j i=-i j$.

No nosso caso, é rotina checar que $A=A_{2}=(K, \alpha, \beta)$, onde $A_{1}=(K, \alpha)=K+K i$ e $A_{2}=A_{1}+A_{1} j$.

A álgebra $A_{0}$ é certamente simples e se $\alpha$ não é um quadrado em $K$, então $A_{1}=(K, \alpha)$ também é simples. De fato, se $\alpha$ não é um quadrado, $A_{1}$ é um corpo, pois é fácil ver que se $a+b \ell \neq 0$, o elemento $\frac{a-b \ell}{a^{2}-\alpha b^{2}}$ é o inverso de $a+b \ell$.

Por outro lado, se $\alpha=\xi^{2}$, para algum $\xi \in K$, então $1 / 2\left(1+\xi^{-1} \ell\right)$ e $1 / 2\left(1-\xi^{-1} \ell\right)$ são idempotentes ortogonais com soma 1 e $(K . \alpha) \cong K \oplus K$.

Analogamente, as álgebras $A_{2}$ e $A_{3}$ são simples.

Temos então a proposição:

Proposição 1.1.10 ([0\%, p.18]) Se $K$ é um corpo de característica $\neq 2$, qualquer álgebra dos quatérnios generalizada $(K, \alpha, \beta)$ é simples e qualquer álgebra de Cayley-Dickson $(\kappa, \alpha, \beta, \gamma)$ é simples.

O seguinte teorema é bem conhecido e nos dá uma caracterização das álgebras alternativas.

Teorema 1.1 .11 (Kleinfeld) ([16, p.151]) Seja A uma álgebra alternativa simples que não é associatica. Então o centro da álgebra Aé um corpo $\epsilon$ A é álgebra de Cayley-Dickson 
sobre seu centro.

Seja $A$ uma álgebra com 1 e $A$ tem involução $a \rightarrow \bar{a}$ tal que $a \bar{a} \in K 1$, para todo $a \in A$.

Se $A$ é álgebra com divisão, ela certamente não tem divisores do zero não triviais. Reciprocamente, se $A$ não tem divisores de zero e $a \in A$ é não nulo, então $a \bar{a}$ é um escalar não nulo e, logo, $a$ é inversível (seu inverso é $\bar{a}(a \bar{a})^{-1}$ ). Em particular, uma álgebra construída pelo processo de Cayley-Dickson é de divisão se, e somente se, ela não tem divisores do zero não triviais.

Definição 1.1.12 Seja $A$ uma álgebra de Cayley-Dickson. A é chamada split se $A$ não é álgebra de divisão.

Nesse caso, $A$ é isomorfa à álgebra matricial-vetorial de Zorn, que estudaremos na próxima seção.

\section{2 Álgebra Matricial-Vetorial de Zorn}

Consideremos o conjunto das matrizes da forma $\left[\begin{array}{ll}a & \mathrm{x} \\ \mathrm{y} & b\end{array}\right]$, onde $a, b \in K$ e x, $\mathrm{y} \in K^{3}$, o conjunto das ternas ordenadas de elementos de $K$.

A adição e a multiplicação por escalar é feita componente a componente e o produto é definido por

$$
\left[\begin{array}{cc}
a_{1} & \mathrm{x}_{1} \\
\mathrm{y}_{1} & b_{1}
\end{array}\right]\left[\begin{array}{cc}
a_{2} & \mathrm{x}_{2} \\
\mathrm{y}_{2} & b_{2}
\end{array}\right]=\left[\begin{array}{cc}
a_{1} a_{2}+\mathrm{x}_{1} \cdot \mathrm{y}_{2} & a_{1} \mathrm{x}_{2}+b_{2} \mathrm{x}_{1}-\mathrm{y}_{1} \wedge \mathrm{y}_{2} \\
a_{2} \mathrm{y}_{1}+b_{1} \mathrm{y}_{2}+\mathrm{x}_{1} \wedge \mathrm{x}_{2} & b_{1} b_{2}+\mathrm{y}_{1} \mathrm{x}_{2}
\end{array}\right]
$$

onde $\mathrm{x} . \mathrm{y}$ e $\mathrm{x} \wedge \mathrm{y}$ denotam, respectivamente. o produto escalar e o produto vetorial em $K^{3}$.

Notação: $\mathcal{Z}(K)$.

Pode-se mostrar que $\mathcal{Z}\left(K^{\prime}\right) \cong(K,-1,-1,1)$

Conseqüentemente, esta é uma álgebra alternativa, que é chamada a álgebra matricialvetorial de Zorn ou a álgebra split de Cayley-Dickson. 
Definição 1.2.1 Dado $\alpha \in \mathcal{Z}\left(K^{\prime}\right)$, dizemos que $\alpha$ é inversível se existe $\beta \in \mathcal{Z}\left(K^{\circ}\right)$ tal que $\alpha \beta=3 \alpha=1$ (aqui 1 denota a matriz identidade). Chamaremos $\beta$ de $\alpha^{-1}$.

Definição 1.2.2 Dado $\alpha \in \mathcal{Z}(K)$ da forma $\left[\begin{array}{ll}a & \mathrm{x} \\ \mathrm{y} & b\end{array}\right]$, definimos determinante de $\alpha$, que denotamos por $\operatorname{det}(\alpha)$, como sendo $\operatorname{det}(\alpha)=a b-\mathrm{x} . \mathrm{y}$. É fácil ver que a aplicação det é multiplicativa [7, p.22]. Pode-se mostrar que $\alpha$ é inversível se, e somente se, $\operatorname{det} \alpha \neq 0$. e vale

$$
\alpha^{-1}=(\operatorname{det} \alpha)^{-1}\left[\begin{array}{cc}
b & -\mathrm{x} \\
-\mathbf{y} & a
\end{array}\right]
$$

O conjunto dos elementos inversíveis de uma álgebra alternativa forma um loop de Moufang que estudaremos no parágrafo seguinte [7, p.81].

\subsection{Loops}

Definição 1.3.1 Um quasigrupo é um par $(L, \cdot)$, onde $L \neq \phi$ e a operação $(a, b) \rightarrow a . b$ é uma operaçào binária sobre $L$ tal que as equações

$$
\begin{aligned}
& a \cdot x=b \\
& x . a=b
\end{aligned}
$$

têm solução única em $L$, para todo $a, b \in L$.

Definição 1.3.2 Um loop é um quasigrupo com elemento identidade 1.

Ambas as regras de cancelamento valem num quasigrupo. Também podemos definir aplicaçōes translaçòes à direita e à esquerda da seguinte maneira:

$$
\begin{aligned}
& \mathcal{R}(x): a \rightarrow a . x \\
& \mathcal{L}(x): a \rightarrow x . a . \quad \forall a \in L .
\end{aligned}
$$


Da definição de quasigrupo, podemos concluir que $\mathcal{R}(x)$ e $\mathcal{L}(x)$ são aplicações bijetoras em $L$. As translações à direita e à esquerda geram um grupo, $\mathcal{M}(L)$, chamado grupo multiplicativo de $L$, que é um subgrupo do grupo das permutações de $L$. Se $(L, \cdot)$ é loop. a equação $a \cdot x=1$ (respectivamente $x . a=1$ ) tem uma única solução $a^{\rho}$ (respectivamente $a^{\lambda}$ ) chamado inverso à direita (respectivamente inverso à esquerda) de a. Em geral. inversos à direita e à esquerda de a podem ser diferentes. Quando forem iguais, usaremos a notação $a^{-1}$.

Definição 1.3.3 Um subloop de um loop $(L, \cdot)$ é um subconjunto não vazio $H$ de $L$ que com a restrição da operação binária de $L$ é também um loop. Como para $h \in H$ a equação $h . x=h$ tem uma única solução em $L$, segue que $1_{H}=1_{L}$. Vamos escrever simplesmente $L$ para denotar um loop, assim como $x y$ ao invés de $x . y$. Vale também:

Proposição 1.3 .4 ([07, p.51]) Seja L um loop e seja $H$ um subconjunto não vazio de L. São equivalentes:

(i) Hé um subloop de L;

(ii) Se $x . y \in H$, então os elementos $x y, x \mathcal{R}(y)^{-1}, x \mathcal{L}(y)^{-1}$ estão todos em $H$;

(iii) Se $x, y, z \in L$ são tais que $x y=z$ e dois dos elementos $x, y, z$ estão em $H$, então o terceiro destes elementos também está em $H$.

Seja $H$ um subloop de um loop finito $L$. Em geral o teorema de Lagrange não vale para loops. Como as regras de cancelamento valem para $L$, temos que $|x H|=|H x|=|H|$, para todo $x \in L$. Assim, se $L$ é a união disjunta de classes laterais de $H$, então $|L|$ é divisivel pela ordem de $H$.

Uma condição necessária e suficiente para que $L$ seja união disjunta de classes laterais à direita é que 
(1) $H x \cap H y \neq \phi, x, y \in L$ se, e somente se, $H x=H y$.

e esta condição é equivalente a

(2) $H(h x)=H x, \forall x \in L \mathrm{e} \forall h \in H$.

$([07$, p.52])

Observação 1.3.5 Quando vale (2), então existe um subconjunto $\tau$ de $L$ chamado transversal de $H$ em $L$, tal que

$$
L=\bigcup_{x \in \tau} H x \quad \text { com } \quad H x \cap H y=\phi, \quad \forall x, y \in \tau .
$$

Definição 1.3.6 Um loop diz-se diassociativo se, para todo par de elementos $x, y \in L$, o subloop $\langle x, y\rangle$, gerado por $x$ e $y$, é associativo.

Definição 1.3.7 Um subloop $H$ de um loop $L$ é normal e escrevemos $H \triangleleft L$ se, para todo $x, y \in L$, tem-se que

$$
H x=x H,(H x) y=H(x y),(x H) y=x(H y) \quad \text { e } \quad y(x H)=(y x) H .
$$

É fácil mostrar que se $H x=x H, \forall x$, então quaisquer duas das três últimas equações implicam na terceira.

Da Definição 1.3.7 e da condiçào (2) para disjunção das classes, vemos que se $H \triangleleft L$, então as classes laterais de $H$ em $L$ são disjuntas.

Seja $H$ um subloop normal do loop $L$, e $x, y, x_{1}, y_{1} \in L$. Se $H x=H x_{1}$ e $H y=H y_{1}$, então

$$
\begin{aligned}
H(x y) & =(H x) y=\left(H x_{1}\right) y=\left(x_{1} H\right) y=x_{1}\left(H y_{1}\right) \\
& =x_{1}\left(y_{1} H\right)=\left(x_{1} y_{1}\right) H
\end{aligned}
$$


Como na teoria de grupos, o conjunto das classes laterais de $H$ forma um loop, chamado loop quociente. denotado por $L / H$,

$$
L / H=\{H x \mid x \in L\}
$$

Esta construção nos permite ver que os subloops normais de $L$ são precisamente os kernels de homomorfismos com domínio $L$.

Definição 1.3.8 Dado um subconjunto $S$ não vazio de um loop $L$, seja $T$ o conjunto de todos os subloops de $L$ que contém $S$, então o subloop $\langle S\rangle=\bigcap_{H \in T} H$ diz-se o subloop de $L$ gerado por $S$. Pode-se ver facilmente que $\langle S\rangle$ é o menor subloop de $L$ que contém $S$.

Definição 1.3.9 Sejam $a, b$ e $c$ três elementos de um loop $L$. O comutador de $a$ e $b$ é o elemento em $L$, denotado por $[a, b]$, que satisfaz a equação

$$
a b=(b a)[a, b]
$$

e o associador de $a, b$ e $c$ em $L$ é o elemento, denotado por $(a, b, c)$, que satisfaz a equação

$$
(a b) c=\{a(b c)\}(a, b, c) .
$$

Se $X, Y$ e $Z$ são subconjuntos de um loop $L$, denotamos por $[X, Y]$ e $(X, Y, Z)$, respectivamente, o conjunto de todos os comutadores $[x, y]$ e todos associadores da forma $(x, y, z)$, onde $x \in X, y \in Y$ e $z \in Z$.

O subloop comutador-associador de um loop $L$ é o subloop $L^{\prime}$ gerado pelo conjunto de todos os comutadores e todos os associadores. Ou seja,

$$
L^{\prime}=\langle(L, L, L),[L, L]\rangle
$$

O núcleo à esquerda de $L$ é o conjunto

$$
\mathcal{N}_{\mathrm{X}}(L)=\{a \in L \mid(a, x, y)=1 \text {. para todos } x, y \in L\},
$$


O núcleo à direita é o conjunto

$$
\mathcal{N}_{\rho}(L)=\{a \in L \mid(x, y, a)=1 \text {, para todos } x, y \in L\},
$$

o núcleo central de $L$ é

$$
\mathcal{N}_{\mu}(L)=\{a \in L \mid(x, a, y)=1, \text { para todos } x, y \in L\}
$$

e o núcleo de $L$ é

$$
\mathcal{N}(L)=\mathcal{N}_{\lambda}(L) \cap \mathcal{N}_{\rho}(L) \cap \mathcal{N}_{\mu}(L)
$$

O centro de $L$ é

$$
Z(L)=\{x \in \mathcal{N}(L) \|[a, x]=1, \text { para todo } a \in L\}
$$

O seguinte resultado é bem conhecido:

Proposição 1.3 .10 ([07, p.53]) Cada um dos núcleos de um loop é um subloop associativo e portanto um grupo. O centro de um loop é um grupo abeliano

É fácil ver que o centro de um loop é um subloop normal.

Definição 1.3.11 Um loop $L$ diz-se simples se $L$ não possui subloops normais próprios.

Definição 1.3.12 Um loop $L$ é um loop com propriedade inversa se todo $x \in L$ tem um único inverso bilateral. que denotamos $x^{-1}$ e satisfaz

$$
x^{-1}(x y)=y \quad \text { e } \quad(y x) x^{-1}=y, \quad \forall x, y \in L .
$$


Proposição 1.3 .13 ([0\%. p.60]) Seja L um loop com propriedade inversa e denotamos por I e J, respectivamente, as aplicações identidade e inversa, isto é, as funções definidas por $I(x)=x$ e $J(x)=x^{-1}, \forall x \in L$. Então:

(i) $J^{2}=I$, isto é, $\left(x^{-1}\right)^{-1}=x, \forall x \in L$;

(ii) $\mathcal{R}(x)^{-1}=\mathcal{R}\left(x^{-1}\right)$ e $\mathcal{L}(x)^{-1}=\mathcal{L}\left(x^{-1}\right), \forall x \in L$;

(iii) $(x y)^{-1}=y^{-1} x^{-1}, \forall x, y \in L$;

(iv) $J \mathcal{L}(x) J=\mathcal{R}\left(x^{-1}\right)$ e $J \mathcal{R}(x) J=\mathcal{L}\left(x^{-1}\right), \forall x \in L$.

Seja $L$ um loop com propriedade inversa e $H$ subloop de $L$. Como $\left\{h^{-1}, h \in H\right\}=H$, segue que a aplicação $H x \rightarrow x^{-1} H$ está bem definida e é bijeção entre a família de classes laterais à direita e à esquerda. Podemos definir índice de $H$ em $L$ como sendo o menor número de classes laterais à direita de $H$ sobre $L$ se este número é finito e infinito caso contrário.

Notação: $[L: H]$.

Se $L$ é união disjunta de classes laterais (à direita), então essa definição coincide com a definição usual da teoria de grupos. Assim, $L$ é a união disjunta de classes laterais à direita de $H$ se, e somente se, $L$ é a união disjunta de classes laterais à esquerda de $H$. Se $L$ é finito, temos então

$$
[L: H]=\frac{|L|}{|H|} .
$$

Teorema 1.3.14 Num loop L as identidades de Moufang definidas no Lema 1.1.2 são todos equivalentes.

Definição 1.3.15 Um loop diz-se um loop de Moufang se ele satisfaz uma e, portanto, todas as identidade de Moufang. 
Teorema 1.3.16 (Moufang) ([0\%, p.68]) Se a,b, c (não necessariamente distintos) são elementos de um loop de Moufang que se associam em alguma ordem, então o subloop gerado por a,b e c é um grupo.

Corolário 1.3.17 Um loop de Moufang é diassociativo.

Segue abaixo a construção de uma classe de loops de Moufang que não são grupos, descobertos por Orin Chein.

\section{O Processo de Chein}

Seja $G$ um grupo nào abeliano e seja $u$ uma indeterminada. Seja $L=G \dot{U} G u$ a união disjunta de $G$ e $G u$ e estendemos a operação binária de $G$ para $L$ seguindo as regras:

$$
\begin{aligned}
g(h u) & =(h g) u \\
(g u) h & =\left(g h^{-1}\right) u \\
(g u)(h u) & =h^{-1} g, \quad \forall g, h \in G .
\end{aligned}
$$

Então $L$ é um loop de Moufang, denotado por $M(G, 2)$ que não é um grupo.

O caso em que $G=S_{3}$, o grupo das simetrias de três letras, então $M\left(S_{3}^{\prime}, 2\right)$ é um loop de Moufang de ordem 12 e é o menor loop de Moufang que não é um grupo. 


\section{Capítulo 2}

\section{O Loop das Unidades da Álgebra de Zorn}

\subsection{A Álgebra de Zorn}

Seja $A=\mathcal{Z}(K)$, onde $K$ é um corpo, a álgebra matricial vetorial de Zorn. Neste capítulo mostraremos que trata-se de uma álgebra irredutível e estudaremos seu loop de unidades. Em particular, exibimos um conjunto de geradores deste loop e também do subloop normal formado pelas matrizes de determinante igual a 1. Finalmente, definimos o loop linear projetivo e mostramos que é um loop simples. Na última seção calculamos as ordens deste loop no caso em que o corpo $K$ é finito.

Definição 2.1.1 Seja $A=\mathcal{Z}\left(K^{\prime}\right)$, onde $K$ é um corpo. Um subconjunto não vazio $L$ de $A$ diz-se um ideal à esquerda de $A$ se $A L \subset L$.

Definição 2.1.2 Dado $x \in A$, definimos o ideal à esquerda gerado por $x$ e denotamos por $\langle x\rangle_{l}$, como sendo o conjunto de todas as somas finitas $\sum_{a_{i} \in A}\left(a_{n} \cdots\left(a_{2}\left(a_{1} x\right)\right) \cdots\right)$. É fácil ver que $\langle x\rangle_{l}$ é o menor ideal à esquerda de $A$ que contém $x$.

Lembramos que uma álgebra $A$ diz-se irredutivel (à esquerda) se não contém ideais (à esquerda) próprios.

Usaremos a notação $\mathbf{i}, \mathbf{j}, \mathbf{k}$ para representar, respectivamente, os vetores $(1,0,0),(0,1,0)$ e $(0.0 .1)$. 
Lema 2.1.3 Dados $e_{11}=\left[\begin{array}{ll}1 & 0 \\ 0 & 0\end{array}\right]$ e $e_{22}=\left[\begin{array}{ll}0 & 0 \\ 0 & 1\end{array}\right]$ em A. tem-se que $\left\langle e_{11}\right\rangle_{l}=\left\langle e_{22}\right\rangle_{l}=A$.

Demonstração: Seja $\alpha \in A, \quad \alpha=\left[\begin{array}{cc}a & \mathrm{x} \\ \mathrm{y} & b\end{array}\right]$, temos

$$
\alpha e_{11}=\left[\begin{array}{ll}
a & \mathrm{x} \\
\mathrm{y} & b
\end{array}\right]\left[\begin{array}{ll}
1 & 0 \\
0 & 0
\end{array}\right]=\left[\begin{array}{ll}
a & 0 \\
\mathrm{y} & 0
\end{array}\right] \in\left\langle e_{11}\right\rangle_{l}
$$

Como $a, \mathrm{y}$ são arbitrários, os elementos $\left[\begin{array}{ll}1 & 0 \\ \mathbf{k} & 0\end{array}\right]$ e $\left[\begin{array}{ll}1 & 0 \\ \mathbf{i} & 0\end{array}\right]$ estão em $\left\langle e_{11}\right\rangle_{l}$. Logo, o elemento $\left[\begin{array}{cc}1 & \mathrm{i} \\ \mathbf{j}+\mathbf{k} & 0\end{array}\right]$ está em $\left\langle e_{11}\right\rangle_{l}$ pois

$$
\left[\begin{array}{cc}
1 & \mathrm{i} \\
\mathrm{j}+\mathrm{k} & 0
\end{array}\right]=\left[\begin{array}{cc}
0 & \mathrm{k} \\
\mathrm{j}+\mathrm{k} & 0
\end{array}\right]\left[\begin{array}{cc}
1 & 0 \\
\mathrm{k} & 0
\end{array}\right]
$$

Como $\left\langle e_{11}\right\rangle_{l}$ é ideal, a soma dos elementos $\left[\begin{array}{cc}1 & \mathbf{i} \\ \mathbf{j}+\mathbf{k} & 0\end{array}\right]$ e $\left[\begin{array}{ll}1 & 0 \\ \mathbf{i} & 0\end{array}\right]$ também está em $\left\langle e_{11}\right\rangle_{l}$. Ou seja,

$$
\left[\begin{array}{cc}
1 & \mathrm{i} \\
\mathrm{j}+\mathrm{k} & 0
\end{array}\right]+\left[\begin{array}{cc}
1 & 0 \\
\mathrm{i} & 0
\end{array}\right]=\left[\begin{array}{cc}
2 & \mathrm{i} \\
\mathrm{i}+\mathrm{j}+\mathrm{k} & 0
\end{array}\right]=\beta \in\left\langle e_{11}\right\rangle_{l} .
$$

Sendo $\beta$ um elemento inversível, temos $\left\langle e_{11}\right\rangle_{l}=A$. Procedendo de maneira análoga, obtemos um elemento inversivel em $\left\langle e_{22}\right\rangle_{l}$ e, conseqüentemente, $\left\langle e_{22}\right\rangle_{l}=A$.

Mostremos que $A$ não tem ideais à esquerda não triviais.

Suponha que exista $L$ ideal de $A, L \neq(0)$, e seja $\alpha=\left[\begin{array}{cc}a & \mathrm{x} \\ \mathrm{y} & b\end{array}\right] \in L, \alpha \neq 0$. Como $\alpha \neq 0$. então pelo menos uma de suas entradas é não nula.

(i) Suponhamos inicialmente que $\mathrm{x}=\left(x_{1}, x_{2}, x_{3}\right) \neq 0$. Sem perda de generalidade, vamos supor que $x_{3} \neq 0$, já que se $x_{3}=0$ então $x_{1}$ ou $x_{2}$ deve ser não nulo e fazendo as mudanças convenientes no procedimento abaixo, obtemos um resultado similar.

Tomando em $A$ os elementos $\beta_{1}=\left[\begin{array}{ll}0 & \mathrm{i} \\ \mathbf{0} & 0\end{array}\right], \beta_{2}=\left[\begin{array}{ll}0 & \mathrm{j} \\ 0 & 0\end{array}\right]$ e $\beta_{3}=\left[\begin{array}{cc}-x_{3}^{-1} & 0 \\ 0 & 0\end{array}\right]$ e efetuando os produtos:

$$
\beta_{1} \alpha=\left[\begin{array}{ll}
0 & \mathrm{i} \\
0 & 0
\end{array}\right]\left[\begin{array}{cc}
a & \mathrm{x} \\
\mathrm{y} & b
\end{array}\right]=\left[\begin{array}{cc}
y_{1} & b \mathrm{i} \\
\mathrm{i} \wedge \mathrm{x} & 0
\end{array}\right] \in L,
$$




$$
\begin{gathered}
\beta_{2}(\beta, \alpha)=\left[\begin{array}{ll}
0 & \mathrm{j} \\
0 & 0
\end{array}\right]\left[\begin{array}{cc}
y_{1} & b \mathrm{i} \\
\mathrm{i} \wedge \mathrm{x} & 0
\end{array}\right]=\left[\begin{array}{ll}
-x_{3} & 0 \\
-b \mathrm{k} & 0
\end{array}\right] \in L, \\
\beta_{3}\left(\beta_{2}(\beta, \alpha)\right)=\left[\begin{array}{cc}
-x_{3}^{-1} & 0 \\
0 & 0
\end{array}\right]\left[\begin{array}{ll}
-x_{3} & 0 \\
-b \mathrm{k} & 0
\end{array}\right]=\left[\begin{array}{ll}
1 & 0 \\
0 & 0
\end{array}\right]=e_{11} \in L .
\end{gathered}
$$

Vemos que $\left\langle e_{11}\right\rangle_{l} \subseteq L$, donde $L=A$.

(ii) Suponhamos agora que $\mathrm{y}=\left(y_{1}, y_{2}, y_{3}\right) \neq 0$. Como antes, sem perda de generalidade, podemos supor que $y_{3} \neq 0$ e consideramos os seguintes elementos de $A$ :

$$
\beta_{1}=\left[\begin{array}{ll}
0 & 0 \\
\mathbf{i} & 0
\end{array}\right], \quad \beta_{2}=\left[\begin{array}{ll}
0 & 0 \\
\mathrm{j} & 0
\end{array}\right], \quad \beta_{3}=\left[\begin{array}{cc}
0 & 0 \\
0 & y_{3}^{-1}
\end{array}\right] .
$$

Calculamos o produto $\beta_{1} \alpha \in L$ :

$$
\beta_{1} \alpha=\left[\begin{array}{ll}
0 & 0 \\
\mathbf{i} & 0
\end{array}\right]\left[\begin{array}{ll}
a & \mathrm{x} \\
\mathrm{y} & b
\end{array}\right]=\left[\begin{array}{cc}
0 & \mathrm{y} \wedge \mathrm{i} \\
a \mathrm{i} & x_{1}
\end{array}\right] .
$$

Agora também $\beta_{2}\left(\beta_{1} \alpha\right) \in L$. Calculando, temos:

$$
\beta_{2}\left(\beta_{1} \alpha\right)=\left[\begin{array}{ll}
0 & 0 \\
\mathbf{j} & 0
\end{array}\right]\left[\begin{array}{cc}
0 & \mathrm{y} \wedge \mathrm{i} \\
a \mathbf{i} & x_{1}
\end{array}\right]=\left[\begin{array}{ll}
0 & a \mathrm{k} \\
0 & y_{3}
\end{array}\right]
$$

E por fim, calculamos $\beta_{3}\left(\beta_{2}\left(\beta_{1} \alpha\right)\right) \in L$.

$$
\beta_{3}\left(\beta_{2}\left(\beta_{1} \alpha\right)\right)=\left[\begin{array}{cc}
0 & 0 \\
0 & y_{3}^{-1}
\end{array}\right]\left[\begin{array}{ll}
0 & a \mathrm{k} \\
0 & y_{3}
\end{array}\right]=\left[\begin{array}{ll}
0 & 0 \\
0 & 1
\end{array}\right]=e_{22} .
$$

Logo, $\left\langle e_{22}\right\rangle_{l} \subseteq L$, donde $L=A$.

(iii) Consideremos agora o caso em que $b \neq 0$ e tomemos

$$
\beta_{1}=\left[\begin{array}{ll}
0 & 0 \\
0 & 1
\end{array}\right], \quad \beta_{2}=\left[\begin{array}{cc}
0 & 0 \\
0 & b^{-1}
\end{array}\right] \text { em } A .
$$


Então.

$$
\beta_{1} \alpha=\left[\begin{array}{ll}
0 & 0 \\
0 & 1
\end{array}\right]\left[\begin{array}{cc}
a & \mathbf{x} \\
\mathbf{y} & b
\end{array}\right]=\left[\begin{array}{ll}
0 & 0 \\
\mathbf{y} & b
\end{array}\right] \in L
$$

e, consequentemente,

$$
\beta_{2}\left(\beta_{1} \alpha\right)=\left[\begin{array}{cc}
0 & 0 \\
0 & b^{-1}
\end{array}\right]\left[\begin{array}{cc}
0 & 0 \\
y & b
\end{array}\right]=\left[\begin{array}{ll}
0 & 0 \\
0 & 1
\end{array}\right]=e_{22} \in L .
$$

Logo, nesse caso também $\left\langle e_{22}\right\rangle_{l} \subseteq L$, donde $A \subseteq L$ e portanto $A=L$.

(iv) Seja $\alpha \in L, \alpha \neq 0$. Já mostramos que se $\mathbf{x}, \mathbf{y}$ ou $b$ são não nulos, então $\langle\alpha\rangle_{l}=A$. Falta considerar apenas o caso em que $\mathrm{x}=\mathrm{y}=0, b=0$ e $a \neq 0$, isto é,

$$
\alpha=\left[\begin{array}{ll}
a & 0 \\
0 & 0
\end{array}\right] \text {. }
$$

Calculamos:

$$
\left[\begin{array}{cc}
a^{-1} & 0 \\
0 & 0
\end{array}\right]\left[\begin{array}{ll}
a & 0 \\
0 & 0
\end{array}\right]=\left[\begin{array}{ll}
1 & 0 \\
0 & 0
\end{array}\right]=e_{11} \in L .
$$

Donde $\left\langle e_{11}\right\rangle_{l} \subseteq L$ e portanto $A \subseteq L$, isto é, $A=L$.

De uma forma análoga pode-se ver que $A$ não contém ideais à direita próprios.

Assim, demonstramos o seguinte:

Teorema 2.1.4 A álgebra $\mathcal{Z}(K)$ é irredutível à esquerda e à direita.

\subsection{O Loop Linear Geral}

Uma álgebra associativa simples que não é de divisão é isomorfa a $M_{n}(D)$, o anel de matrizes de ordem $n$ sobre $D$, um anel com divisão. Os elementos inversíveis de $M_{n}(D)$ formam um subgrupo, denotado por $G L_{n}(D)$, o Grupo Linear Geral.

Já vimos que uma álgebra alternativa simples que não é de divisão é isomorfa a $\mathcal{Z}(K)$. O conjunto dos elementos inversíveis de $\mathcal{Z}(K)$ é a generalização natural de grupos lineares ao contexto alternativo. 
Definição 2.2.1 O Loop Linear Geral é o loop dos elementos inversíveis da álgebra de Zorn. $\mathcal{Z}(K)$ e é denotado por $G L L(K)$.

Definição 2.2.2 O Loop Linear Especial é o subloop de $G L L(K)$ consistindo de todas as matrizes de determinante 1 e é denotado por $S L L(K)$.

Considere em $\mathcal{Z}\left(K^{\prime}\right)$ os elementos

$$
\begin{aligned}
& e_{11}=\left[\begin{array}{ll}
1 & 0 \\
0 & 0
\end{array}\right], \quad e_{22}=\left[\begin{array}{ll}
0 & 0 \\
0 & 1
\end{array}\right] \\
& e_{12}^{(\mathrm{i})}=\left[\begin{array}{ll}
0 & \mathrm{i} \\
\mathbf{0} & 0
\end{array}\right], \quad e_{12}^{(\mathrm{j})}=\left[\begin{array}{ll}
0 & \mathrm{j} \\
0 & 0
\end{array}\right], \quad e_{12}^{(\mathbf{k})}=\left[\begin{array}{cc}
0 & \mathrm{k} \\
0 & 0
\end{array}\right], \\
& e_{21}^{(\mathrm{i})}=\left[\begin{array}{ll}
0 & 0 \\
\mathrm{i} & 0
\end{array}\right], \quad e_{21}^{(\mathrm{j})}=\left[\begin{array}{ll}
0 & 0 \\
\mathrm{j} & 0
\end{array}\right], \quad e_{21}^{(\mathrm{k})}=\left[\begin{array}{cc}
0 & 0 \\
\mathrm{k} & 0
\end{array}\right] .
\end{aligned}
$$

Lema 2.2.3 Os elementos $e_{i i}, e_{i j}^{(\mathbf{h})}$, onde $i, j=1,2$ e $\mathbf{h}=\mathbf{i}, \mathbf{j}$ ou $\mathbf{k}$, satisfazem as relações:

a) $e_{i i}^{2}=e_{i i}, e_{i i} e_{j j}=0$;

b) $e_{i i} e_{i j}^{(\mathbf{h})}=e_{i j}^{(\mathbf{h})} e_{j j}=e_{i j}^{(\mathbf{h})}, \quad e_{j j} e_{i j}^{(\mathbf{h})}=e_{i j}^{(\mathbf{h})} e_{i i}=0$;

c) $\left(e_{i j}^{(\mathbf{h})}\right)^{2}=0=e_{i j}^{(\mathbf{h})} e_{i j}^{(\mathbf{l})}, \quad \mathbf{h} \neq \mathbf{l}$

d) $\epsilon_{i j}^{(\mathbf{h})} e_{j i}^{(\mathbf{h})}=e_{i i}, \quad e_{i j}^{(\mathbf{h})} e_{j i}^{(\mathbf{l})}=0, \quad \mathbf{h} \neq 1:$

e) $e_{i j}^{(\mathrm{h})} e_{i j}^{(\mathrm{l})}=\left\{\begin{array}{l}e_{12}^{(\mathrm{h})} e_{12}^{(1)}=e_{21}^{(\mathrm{h} \wedge \mathrm{l})} \\ e_{21}^{(\mathrm{h})} e_{21}^{(1)}=e_{12}^{-(\mathrm{h} \wedge 1)}\end{array}\right.$

Demonstração: Imediata, por comprovação direta.

Definição 2.2.4 Dado $\lambda \in K$, definimos uma transveç̧ão em $G L L\left(K^{\circ}\right)$ e denotamos por $\tau_{i j}^{(\mathbf{h})}(\lambda)$, onde $i, j=1,2, i \neq j \mathrm{e} \mathbf{h}=\mathbf{i}, \mathbf{j}$ ou $\mathbf{k}$, como sendo o elemento

$$
\tau_{i j}^{(\mathbf{h})}(\lambda)=I+\lambda e_{i j}^{(\mathbf{h})} \in G L L\left(K^{\prime}\right),
$$


onde $I$ é a matriz identidade em $\mathcal{Z}(K)$ e $e_{i j}^{(\mathrm{h})}$ são as matrizes definidas acima.

Por exemplo, $\tau_{12}^{(\mathbf{j})}(\lambda)=\left[\begin{array}{cc}1 & (0, \lambda, 0) \\ 0 & 1\end{array}\right]$.

Como a aplicação det : $G L L\left(K^{*}\right) \rightarrow K^{*}=K \backslash\{0\}$ é um homomorfismo e Ker(det) = $S L L(K)$, temos o seguinte:

Lema 2.2.5 $S L L(K) \triangleleft G L L(K)$.

Lema 2.2.6 a) $Z(G L L(K))=K^{*} I$;

b) $Z(S L L(K))=\{I,-I\}$.

\section{Demonstração:}

a) Para simplificar a notação, chamemos $G=G L L(K)$.

Seja $\alpha=\left[\begin{array}{ll}a & \mathrm{x} \\ \mathrm{y} & b\end{array}\right] \in \mathcal{Z}(G)$ e $\beta=\left[\begin{array}{cc}c & \mathrm{v} \\ \mathbf{u} & d\end{array}\right] \in G$ um elemento qualquer.

Calculemos $\alpha \beta$ e também $\beta \alpha$.

$$
\begin{aligned}
& {\left[\begin{array}{ll}
a & \mathrm{x} \\
\mathrm{y} & b
\end{array}\right]\left[\begin{array}{ll}
c & \mathrm{v} \\
\mathrm{u} & d
\end{array}\right]=\left[\begin{array}{cc}
a c+\mathrm{x} \cdot \mathrm{u} & a \mathrm{v}+d \mathrm{x}-\mathrm{y} \wedge \mathrm{u} \\
c \mathrm{y}+b \mathrm{u}+\mathrm{x} \wedge \mathrm{v} & b d+\mathrm{y} \cdot \mathbf{v}
\end{array}\right],} \\
& {\left[\begin{array}{ll}
c & \mathrm{v} \\
\mathrm{u} & d
\end{array}\right]\left[\begin{array}{ll}
a & \mathrm{x} \\
\mathrm{y} & b
\end{array}\right]=\left[\begin{array}{cc}
c a+\mathrm{v} \cdot \mathrm{y} & c \mathbf{x}+b \mathbf{v}-\mathbf{u} \wedge \mathrm{y} \\
a \mathbf{u}+d \mathbf{y}+\mathrm{v} \wedge \mathrm{x} & d b+\mathrm{x} \cdot \mathbf{u}
\end{array}\right] .}
\end{aligned}
$$

Como $\alpha \beta=\beta \alpha, \forall \beta \in G$, devemos ter $\mathbf{x} \cdot \mathbf{u}=\mathrm{v} \cdot \mathrm{y}, \quad \forall \mathbf{u}, \mathrm{v}$. Tomemos $\mathrm{u}=\mathrm{x} \quad \mathrm{e}$ $\mathbf{v}=0$. Então temos que $\mathrm{x} \cdot \mathrm{x}=0$, donde $\mathrm{x}=0$. De forma análoga, segue que $\mathrm{y}=0$.

Agora, temos que

$$
\begin{aligned}
a \mathbf{v} & =b \mathbf{v}, \\
b \mathrm{u} & =a \mathbf{u}, \quad \forall \mathrm{u} . \mathbf{v} \Rightarrow a=b .
\end{aligned}
$$


Conseqüentemente,

$$
\alpha \in Z(G) \Rightarrow a=\left[\begin{array}{ll}
a & 0 \\
0 & a
\end{array}\right] \quad \text { ou } \quad \alpha=a I, a \in K^{*} .
$$

É claro que $K^{*} I \subseteq Z(G)$, donde $\mathcal{Z}(G)=K^{*} I$.

b) Como antes, chamemos $S L L\left(K^{\circ}\right)=S$.

Seja $\alpha \in Z(S), \beta$ elemento qualquer em $S, \alpha=\left[\begin{array}{cc}a & \mathrm{x} \\ \mathrm{y} & b\end{array}\right] ; \beta=\left[\begin{array}{cc}c & \mathrm{v} \\ \mathrm{u} & d\end{array}\right]$.

Devemos ter $\alpha \beta=\beta \alpha$ e ainda $a b-\mathrm{x} \cdot \mathrm{y}=1=c d-\mathrm{u} . \mathrm{v}$.

Portanto, $\quad \mathbf{x} \cdot \mathrm{u}=\mathrm{v} \cdot \mathrm{y}, \forall \mathrm{u}, \mathrm{v}$ e como antes, segue que $\mathrm{x}=\mathrm{y}=\mathbf{0}$.

Devemos ter ainda:

$$
\begin{aligned}
& a \mathbf{v}=b \mathbf{v}, \\
& a \mathbf{u}=b \mathbf{u}, \forall \mathbf{u}, \mathbf{v},
\end{aligned}
$$

o que implica $a=b$.

Logo, $\alpha \in Z(S) \Rightarrow \alpha=\left[\begin{array}{ll}a & 0 \\ 0 & a\end{array}\right], \operatorname{com} a^{2}=\operatorname{det} \alpha=1$.

Como $a \in K^{*}$, então $a^{2}-1=0 \Rightarrow(a-1)(a+1)=0$, portanto,

$$
a=1 \quad \text { ou } \quad a=-1 .
$$

Assim, $Z(S)=\{I,-I\}$.

Se denotamos $\left(G L L\left(K^{\prime}\right)\right)^{\prime}$, o subloop comutador-associador de $G L L(K)$, então valem os seguintes resultados:

Proposição 2.2.7 ([07, p.56]) $\left(G L L\left(K^{\prime}\right)\right)^{\prime} \triangleleft G L L\left(K^{\prime}\right)$

O loop quociente $G L L\left(K^{\circ}\right) /\left(G L L\left(K^{\prime}\right)\right)^{\prime}$ é o conjunto dos elementos da forma $x\left(G L L\left(K^{\circ}\right)\right)^{\prime}$, $x \in G L L\left(K^{\circ}\right)$ com o produto dado por

$$
x\left(G L L\left(K^{-}\right)\right)^{\prime} y\left(G L L\left(K^{\prime}\right)\right)^{\prime}=x y\left(G L L\left(K^{\prime}\right)\right)^{\prime}
$$


Lema 2.2.8 ([03]) O loop quociente $G L L\left(K^{\prime}\right) /\left(G L L\left(K^{*}\right)\right)^{\prime}$ é associativo e comutativo

O seguinte lema é muito simples mas será usado repetidamente no que segue.

Lema 2.2.9 Seja L um loop de Moufang e sejam $\alpha, \beta, \gamma \in L$. Se $\alpha \beta=\gamma$, então $\alpha=$ $\gamma \beta^{-1}$.

Demonstração: Como $\alpha \beta=\gamma$, multiplicando ambos os lados da equação por $\beta^{-1}$, temos que $(\alpha \beta) \beta^{-1}=\gamma \beta^{-1}$.

Como $(\alpha \beta) \beta^{-1} \in\langle\alpha, \beta\rangle$, pela diassociatividade tem-se que $(\alpha \beta) \beta^{-1}=\alpha\left(\beta \beta^{-1}\right)=\alpha$ e segue a tese.

Lema 2.2.10 Se $\alpha$ é um elemento da forma $\alpha=\left[\begin{array}{ll}1 & 0 \\ \mathrm{v} & 1\end{array}\right]$, então $\alpha$ pode ser escrito como um produto de transveç̧ões.

Demonstração: Seja $\mathbf{v}=\left(v_{1}, v_{2}, v_{3}\right)$ e considere $\gamma=\tau_{12}^{(\mathbf{i})}\left(v_{2} v_{3}\right) \tau_{21}^{(\mathbf{j})}\left(-v_{2}\right)$. Ou seja,

$$
\gamma=\left[\begin{array}{cc}
1 & v_{2} v_{3} \mathbf{i} \\
0 & 1
\end{array}\right]\left[\begin{array}{cc}
1 & 0 \\
-v_{2} \mathbf{j} & 1
\end{array}\right]=\left[\begin{array}{cc}
1 & v_{2} v_{3} \mathbf{i} \\
-v_{2} \mathbf{j} & 1
\end{array}\right] .
$$

Temos que

$$
\begin{aligned}
\alpha \gamma & =\left[\begin{array}{ll}
1 & 0 \\
\mathbf{v} & 1
\end{array}\right]\left[\begin{array}{cc}
1 & v_{2} v_{3} \mathbf{i} \\
-v_{2} \mathbf{j} & 1
\end{array}\right]=\left[\begin{array}{cc}
1 & v_{2} v_{3} \mathbf{i}+v_{2}(\mathbf{v} \wedge \mathbf{j}) \\
\mathbf{v}-v_{2} \mathbf{j} & 1+v_{1} v_{2} v_{3}
\end{array}\right] \\
& =\left[\begin{array}{cc}
1 & \left(0,0, v_{1} v_{2}\right) \\
\left(v_{1}, 0, v_{3}\right) & 1+v_{1} v_{2} v_{3}
\end{array}\right] .
\end{aligned}
$$

Agora, calculamos $\alpha_{2}=(\alpha \gamma)\left(\tau_{12}^{(\mathbf{k})}\left(-v_{1} v_{2}\right)\right)$ :

$$
\left[\begin{array}{cc}
1 & \left(0,0, v_{1} v_{2}\right) \\
\left(v_{1}, 0, v_{3}\right) & 1+v_{1} v_{2} v_{3}
\end{array}\right]\left[\begin{array}{cc}
1 & -v_{1} v_{2} \mathrm{k} \\
0 & 1
\end{array}\right]=\left[\begin{array}{cc}
1 & 0 \\
\left(v_{1}, 0, v_{3}\right) & 1
\end{array}\right] .
$$

Consideremos agora o produto $\alpha_{3}=a_{2} \tau_{21}^{(\mathrm{i})}\left(-v_{1}\right)$ :

$$
\left[\begin{array}{cc}
1 & 0 \\
\left(v_{1}, 0, v_{3}\right) & 1
\end{array}\right]\left[\begin{array}{cc}
1 & 0 \\
-v_{1} \mathbf{i} & 1
\end{array}\right]=\left[\begin{array}{cc}
1 & -v_{1} v_{3} \mathbf{j} \\
v_{3} \mathrm{k} & 1
\end{array}\right] .
$$


Finalmente, calculamos $\alpha_{4}=\alpha_{3} \tau_{12}^{(\mathbf{j})}\left(v_{1} v_{3}\right)$ :

$$
\left[\begin{array}{cc}
1 & -v_{1} v_{3} \mathbf{j} \\
v_{3} \mathbf{k} & 1
\end{array}\right]\left[\begin{array}{cc}
1 & v_{1} v_{3} \mathbf{j} \\
0 & 1
\end{array}\right]=\left[\begin{array}{cc}
1 & 0 \\
v_{3} \mathbf{k} & 1
\end{array}\right]
$$

Temos assim que

$$
\left[\left((\alpha \gamma) \tau_{12}^{(\mathbf{k})}\left(-v_{1} v_{2}\right) \tau_{21}^{(\mathbf{i})}\left(-v_{1}\right)\right] \tau_{12}^{(\mathbf{j})}\left(v_{1} v_{3}\right)=\tau_{21}^{(\mathbf{k})}\left(v_{3}\right)\right.
$$

Logo.

$$
\alpha=\left[\left(\left(\tau_{21}^{(\mathbf{k})}\left(v_{3}\right) \cdot \tau_{12}^{(\mathbf{j})}\left(-v_{1} v_{3}\right)\right) \tau_{21}^{(\mathbf{i})}\left(v_{1}\right)\right) \tau_{12}^{(\mathbf{k})}\left(v_{1} v_{2}\right)\right]\left[\tau_{12}^{(\mathbf{i})}\left(-v_{2} v_{3}\right) \cdot \tau_{21}^{(\mathbf{j})}\left(v_{2}\right)\right]
$$

Definição 2.2.11 Dado $\mu \in K, \mu \neq 0$, definimos $D(\mu) \in G L L(K)$ como sendo o elemento

$$
D(\mu)=\left[\begin{array}{ll}
1 & 0 \\
0 & \mu
\end{array}\right]
$$

Teorema 2.2.12 Com a notação acima,

$$
\begin{aligned}
& G L L(K)=\left\langle\tau_{i j}^{(\mathbf{h})}(\lambda), D(\mu) \mid \mathbf{h}=\mathbf{i}, \mathbf{j}, \mathbf{k}, \mu \neq 0, \lambda, \mu \in K\right\rangle \\
& S L L(K)=\left\langle\tau_{i j}^{(\mathbf{h})}(\lambda) \mid \mathbf{h}=\mathbf{i}, \mathbf{j}, \mathbf{k}, \lambda \in K\right\rangle .
\end{aligned}
$$

Demonstração: Seja $\alpha \in G L L\left(K^{\prime}\right), \alpha=\left[\begin{array}{cc}a & \mathrm{x} \\ \mathrm{y} & b\end{array}\right]$. Como $\operatorname{det} \alpha \neq 0$, podemos supor então que $\mathrm{x} \neq 0$. De fato, em caso contrário deveremos ter $a, b \neq 0$ e multiplicando por $\tau_{12}^{(i)}(\lambda)$ ficamos com

$$
\left[\begin{array}{ll}
a & 0 \\
\mathbf{y} & b
\end{array}\right]\left[\begin{array}{cc}
1 & \lambda \mathbf{i} \\
0 & 1
\end{array}\right]=\left[\begin{array}{cc}
a & \lambda a \mathbf{i} \\
\mathbf{y} & b+\lambda y_{1}
\end{array}\right]=\alpha^{\prime} \in \operatorname{GLL}(K)
$$

Nesta matriz de $G L L\left(K^{\prime}\right)$. o vetor que ocupa a posição $(1.2)$ é $\mathrm{x}^{\prime}=\lambda a \mathrm{i} \neq 0$. 
Considere então $\mathbf{x} \neq \mathbf{0}, \mathbf{x}=\left(x_{1}, x_{2}, x_{3}\right)$. Alguma de suas coordenadas é não nula. Suponha $x_{1} \neq 0$. Se $x_{1}=0$, então $x_{2}$ ou $x_{3}$ é não nulo e fazemos as mudanças correspondentes.

Multiplicando $\alpha$ à direita por $\tau_{21}^{(\mathrm{i})}(\lambda)$ :

$$
\left[\begin{array}{cc}
a & \mathrm{x} \\
\mathrm{y} & b
\end{array}\right]\left[\begin{array}{cc}
1 & 0 \\
\lambda \mathrm{i} & 1
\end{array}\right]=\left[\begin{array}{cc}
a+\lambda x_{1} & \mathrm{x}-\lambda \mathrm{y} \wedge \mathrm{i} \\
\mathrm{y}+\lambda b \mathrm{i} & b
\end{array}\right]=\alpha_{1} .
$$

Tomando $\lambda=\frac{1-a}{x_{1}}, \alpha_{1}$ terá a forma $\left[\begin{array}{ll}1 & \mathrm{u} \\ \mathrm{v} & b\end{array}\right]$, onde

$$
\begin{aligned}
& \mathbf{u}=(\underbrace{x_{1}}_{u_{1}}, \underbrace{x_{2}-\lambda y_{3}}_{u_{2}}, \underbrace{x_{3}+\lambda y_{2}}_{u_{3}}) \\
& \mathbf{v}=(\underbrace{y_{1}+\lambda b}_{v_{1}}, \underbrace{y_{2}}_{v_{2}}, \underbrace{y_{3}}_{v_{3}})
\end{aligned}
$$

Se $u_{3} \neq 0$, vamos provar que existe uma transvecção $\tau_{1}=\tau_{12}^{(\mathbf{k})}\left(-u_{3}\right)$ tal que $\alpha_{2}=\alpha_{1} \tau$ é da forma $\alpha_{2}=\left[\begin{array}{cc}1 & \left(u_{1}, u_{2}, 0\right) \\ \mathbf{v}^{\prime} & b_{1}\end{array}\right]$.

Com efeito, temos que:

$$
\begin{aligned}
\alpha_{2} & =\alpha_{1} \tau_{12}^{(\mathbf{k})}\left(-u_{3}\right) \\
& =\left[\begin{array}{ll}
1 & \mathbf{u} \\
\mathbf{v} & b
\end{array}\right]\left[\begin{array}{cc}
1 & -u_{3} \mathbf{k} \\
0 & 1
\end{array}\right]=\left[\begin{array}{cc}
1 & \left(u_{1}, u_{2}, 0\right) \\
\mathbf{v}-u_{3}(\mathbf{u} \wedge \mathbf{k}) & b-u_{3} v_{3}
\end{array}\right] \\
& =\left[\begin{array}{cc}
1 & \left(u_{1}, u_{2}, 0\right) \\
\underbrace{\mathbf{v}-\left(u_{2} u_{3},-u_{1} u_{3}, 0\right)}_{\mathbf{v}^{\prime}} & \underbrace{b-u_{3} v_{3}}_{b_{1}}
\end{array}\right]
\end{aligned}
$$

Novamente, se $u_{2} \neq 0$, mostremos que existe uma transvecção $\tau_{2}=\tau_{12}^{(\mathbf{j})}\left(-u_{2}\right)$ tal que $\alpha_{3}=\alpha_{2} \tau$ é da forma $\alpha_{3}=\left[\begin{array}{cc}1 & \left(u_{1}, 0,0\right) \\ \mathbf{v}^{\prime \prime} & b_{2}\end{array}\right]$.

De fato. $\alpha_{3}=\alpha_{2} \tau_{12}^{(\mathrm{j})}\left(-u_{2}\right)$,

$$
\alpha_{3}=\left[\begin{array}{cc}
1 & \left(u_{1}, u_{2}, 0\right) \\
\mathbf{v}^{\prime} & b-u_{3} v_{3}
\end{array}\right]\left[\begin{array}{cc}
1 & -u_{2} \mathbf{j} \\
0 & 1
\end{array}\right]
$$




$$
\begin{gathered}
=\left[\begin{array}{cc}
1 & u_{1} \mathrm{i} \\
\mathrm{v}^{\prime \prime} \underbrace{b-u_{1} u_{2} u_{3}-u_{2} v_{2}-u_{3} v_{3}}_{b_{2}}
\end{array}\right], \\
\mathrm{v}^{\prime \prime}=\mathrm{v}^{\prime}+\left(0,0,-u_{1} u_{2}\right)=\left(v_{1}-u_{2} u_{3}, v_{2}+u_{1} u_{3}, v_{3}-u_{1} u_{2}\right) .
\end{gathered}
$$

Finalmente, se $u_{1} \neq 0$, provamos que existe uma transvecção $\tau_{3}=\tau_{12}^{(\mathbf{i})}\left(-u_{1}\right)$ tal que $\alpha_{4}=\alpha_{3} \tau$ é da forma $\alpha_{4}=\left[\begin{array}{cc}1 & 0 \\ \mathrm{v}^{\prime \prime} & b_{3}\end{array}\right]$ :

$$
\begin{aligned}
& \alpha_{4}=\left[\begin{array}{cc}
1 & u_{1} \mathbf{i} \\
\mathbf{v}^{\prime \prime} & b_{2}
\end{array}\right]\left[\begin{array}{cc}
1 & -u_{1} \mathbf{i} \\
0 & 1
\end{array}\right] \\
& =\left[\begin{array}{cc}
1 & 0 \\
\mathrm{v}^{\prime \prime} & \underbrace{b_{2}-u_{1}\left(v_{1}-u_{2} u_{3}\right)}_{b_{3}}
\end{array}\right] \text {, }
\end{aligned}
$$

onde

$$
\begin{aligned}
b_{3} & =b_{2}-u_{1}\left(v_{1}-u_{2} u_{3}\right)=b_{2}-u_{1} v_{1}+u_{1} u_{2} u_{3} \\
& =b-u_{1} u_{2} u_{3}-u_{2} v_{2}-u_{3} v_{3}-u_{1} v_{1}+u_{1} u_{2} u_{3}=b-\mathbf{u} \cdot \mathbf{v} \\
\mathbf{u} & =\left(x_{1}, x_{2}-\lambda y_{3}, x_{3}+\lambda y_{2}\right) \\
\mathbf{v} & =\left(y_{1}+\lambda b, y_{2}, y_{3}\right) \\
\mathbf{u} . \mathbf{v} & =x_{1} y_{1}+\lambda b x_{1}+x_{2} y_{2}-\lambda y_{2} y_{3}+x_{3} y_{3}+\lambda y_{2} y_{3} \\
& =\mathrm{x} \cdot \mathrm{y}+\frac{(1-a)}{x_{1}} b x_{1}=\mathrm{x} \cdot \mathrm{y}+b-a b
\end{aligned}
$$

Assim, $b_{3}=b-(\mathbf{x} \cdot \mathrm{y}+b-a b)=a b-\mathbf{x} \cdot \mathbf{y}=\operatorname{det} \alpha$.

Com isso, dado $\alpha=\left[\begin{array}{ll}a & \mathrm{x} \\ \mathrm{y} & b\end{array}\right]$, através de multiplicações à direita por transvecções. obtemos uma matriz $\beta$ da forma

$$
\beta=\left[\begin{array}{ll}
1 & 0 \\
\mathbf{z} & \mu
\end{array}\right], \quad \text { onde } \mu=\operatorname{det} \alpha
$$


Agora, tomando $\gamma=\left[\begin{array}{cc}1 & 0 \\ -\mu^{-1} \mathrm{z} & 1\end{array}\right]$, temos que

$$
\beta \gamma=\left[\begin{array}{ll}
1 & 0 \\
\mathbf{z} & \mu
\end{array}\right]\left[\begin{array}{cc}
1 & 0 \\
-\mu^{-1} \mathbf{z} & 1
\end{array}\right]=\left[\begin{array}{ll}
1 & 0 \\
0 & \mu
\end{array}\right] \text {. }
$$

Logo, temos mostrado que

$$
\begin{gathered}
\left(\left(\left(\alpha \tau_{1}\right) \tau_{2}\right) \tau_{3}\right) \gamma=\left[\begin{array}{ll}
1 & 0 \\
0 & \mu
\end{array}\right]=D(\mu), \\
\alpha=\left(\left(\left(D(\mu), \gamma^{-1}\right) \tau_{3}^{-1}\right) \tau_{2}^{-1}\right) \tau_{1}^{-1} .
\end{gathered}
$$

Como $\gamma$ é produto de transvecções, segue que

$$
\alpha \in\left\langle\tau_{i j}^{(\mathbf{h})}, D(\mu) \mid \mathbf{h}=\mathbf{i}, \mathbf{j}, \mathbf{k} ; i, j=1,2 ; \lambda, \mu \in K\right\rangle .
$$

A demonstração anterior mostra que qualquer matriz tendo determinante 1 pode ser escrita na forma $\tau_{i j}^{(\mathbf{h})}(\lambda)$, pois se $\alpha \in S L L(K)$, pela demonstração acima temos $D(\mu)=I$.

\section{$2.3 \quad$ A Simplicidade de $P S L L(K)$}

Nesta seção mostraremos que $\operatorname{PSLL}(K)$ é simples. Este resultado foi obtido pela primeira vez por L. Paige [10].

Nossa demonstração é diferente e utiliza o conjunto de geradores introduzido na seção anterior, que é bem menor que o considerado originariamente por Paige.

Definição 2.3.1 Se $Z_{0}$ denota o centro de $S L L(K)$, definimos o Loop Linear Projetivo e denotamos por PSLL( $\left.K^{\circ}\right)$, como sendo o loop quociente:

$$
\operatorname{PSLL}\left(K^{\circ}\right)=\frac{S^{\prime} L L\left(K^{\circ}\right)}{Z_{0}} \text {. }
$$


Nosso objetivo é demonstrar que $P S L L(K)$ é simples.

Definição 2.3.2 Sejam x e y elementos de um loop $L$. Definimos bijeções $\mathcal{T}(x), \mathcal{R}(x, y)$ e $\mathcal{L}(x, y)$ por

$$
\begin{aligned}
\mathcal{T}(x) & =\mathcal{R}(x) \mathcal{L}(x)^{-1}, \\
\mathcal{R}(x, y) & =\mathcal{R}(x) \mathcal{R}(y) \mathcal{R}(x y)^{-1}, \\
\mathcal{L}(x, y) & =\mathcal{L}(x) \mathcal{L}(y) \mathcal{L}(y x)^{-1} .
\end{aligned}
$$

Evidentemente, cada uma destas aplicações estão no grupo multiplicativo $\mathcal{M}(L)$. O grupo dos automorfismos internos de $L$ é o subgrupo $\operatorname{Inn}(L)$ de $\mathcal{M}(L)$ gerado por todas as aplicações da forma $\mathcal{T}(x), \mathcal{R}(x, y), \mathcal{L}(x, y)$,

$$
\operatorname{Inn}(L)=\langle\mathcal{T}(x), \mathcal{R}(x, y), \mathcal{L}(x, y) \mid x, y \in L\rangle
$$

e os elementos de $\operatorname{Inn}(L)$ são chamados aplicações internas.

Observando que $H x=x H$ se e somente se $H \mathcal{T}(x)=H$, que $(H x) y=H(x y)$ se e somente se $H \mathcal{R}(x, y)=H$ e que $y(x H)=(y x) H$ se e somente se $H \mathcal{L}(x, y)=H$, vemos que $H$ é um subloop normal de $L$ se e somente se $\theta(H)=H$ para todo $\theta \in \operatorname{Inn}(L)$.

Definição 2.3.3 Dois elementos $x, y \in L$ dizem-se conjugados se e somente se existe $\theta \in \operatorname{Inn}(L)$ tal que $\theta(x)=y$.

Observação 2.3.4 Em particular, note que, dados $x, y \in L$ e $a \in L$, o produto $a^{-1} x a$ está bem definido, pois $H$ é diassociativo e dizer que $a^{-1} x a=y$ é equivalente a escrever $\mathcal{T}(a)(x)=y$.

Lema 2.3.5 Duas transveções são conjugadas $\operatorname{tm} S L L\left(K^{\prime}\right)$.

Vamos dividir a demonstração em cinco casos: 
i) $\tau_{12}^{(\mathbf{h})}(\lambda)$ é conjugada a $\tau_{12}^{(\mathbf{f})}(\rho)$ para quaisquer $\lambda . \rho$ não nulos em $K ; \mathbf{h}, \mathbf{f}=\mathrm{i}, \mathbf{j}$ ou $\mathbf{k}$. com $h \neq f$.

Demonstração: Seja $\mathbf{u} \in K^{3}$ um vetor tal que $\mathbf{u} \cdot \mathbf{h}=-1$ e $\mathbf{u} \cdot \mathbf{f}=-\rho^{-1}$. Conjugando

$$
\left[\begin{array}{cc}
0 & \rho \mathbf{f} \\
\mathbf{u} & 0
\end{array}\right]\left(\left[\begin{array}{cc}
0 & \lambda \mathbf{h} \\
\mathbf{u} & 0
\end{array}\right]\left[\begin{array}{cc}
1 & \lambda \mathbf{h} \\
\mathbf{0} & 1
\end{array}\right]\left[\begin{array}{cc}
0 & -\mathbf{h} \\
-\frac{\mathbf{u}}{\lambda} & 0
\end{array}\right]\right)\left[\begin{array}{cc}
0 & -\rho \mathbf{f} \\
-\mathbf{u} & 0
\end{array}\right]=\left[\begin{array}{cc}
1 & \rho \mathbf{f} \\
\mathbf{0} & 1
\end{array}\right]
$$

ii) $\tau_{21}^{(\mathbf{h})}(\lambda)$ é conjugada a $\tau_{21}^{(\mathbf{f})}(\rho)$ para quaisquer $\lambda, \rho$ não nulos em $K ; \mathbf{h}, \mathbf{f}=\mathbf{i}, \mathbf{j}$ ou $\mathbf{k}$, com $h \neq f$.

A demonstração é análoga ao caso anterior.

iii) $\tau_{12}^{(\mathbf{h})}(\lambda)$ é conjugada a $\tau_{21}^{(\mathbf{h})}(\rho)$ para qualquer $\lambda, \rho$ não nulos em $K ; \mathbf{h}=\mathbf{i}, \mathbf{j}$ ou $\mathbf{k}$.

Demonstração: Conjugando

$$
\left[\begin{array}{cc}
0 & \frac{\mathbf{h}}{\rho} \\
\frac{\mathbf{h}}{\lambda} & 0
\end{array}\right]\left[\begin{array}{cc}
1 & \lambda \mathbf{h} \\
\mathbf{0} & 1
\end{array}\right]\left[\begin{array}{cc}
0 & \lambda \mathbf{h} \\
\rho \mathbf{h} & 0
\end{array}\right]=\left[\begin{array}{cc}
1 & \mathbf{0} \\
\rho \mathbf{h} & 1
\end{array}\right] .
$$

iv) $\tau_{12}^{(\mathbf{h})}(\lambda)$ é conjugada a $\tau_{12}^{(\mathbf{h})}(\rho)$ para quaisquer $\lambda, \rho$ não nulos em $K ; \mathbf{h}=\mathbf{i}, \mathbf{j}$ ou $\mathbf{k}$.

Demonstração: Já vimos no caso (i) que $\tau_{12}^{(\mathbf{h})}(\lambda)$ é conjugada a $\tau_{12}^{(\mathbf{f})}(\rho)$ para $\mathbf{h} \neq \mathbf{f}$. Ainda. pelo caso (i), temos $\tau_{12}^{(\mathbf{f})}(\rho)$ é conjugada a $\tau_{12}^{(\mathbf{h})}(\rho)$.

v) $\tau_{21}^{(\mathbf{h})}(\lambda)$ é conjugada a $\tau_{21}^{(\mathbf{h})}(\rho)$ para quaisquer $\lambda, \rho$ não nulos em $K ; \mathbf{h}=\mathbf{i}, \mathbf{j}$ ou $\mathbf{k}$.

Novamente, a demonstração é análoga ao caso anterior.

A partir destas verificações a tese segue imediatamente.

No que segue, $H$ denotará sempre um subloop normal de $S L L(K), H \neq(1)$, que contém $Z_{0}$. Mostraremos que se $H \neq Z_{0}$, então $H=S L L\left(K^{\prime}\right)$. Isto será feito mostrando que $H$ contém uma, e portanto, todas as transvecçòes. 
Lema 2.3.6 Se $H$ contém um elemento não central da forma

$$
\alpha=\left[\begin{array}{cc} 
\pm 1 & \mathrm{x} \\
0 & \pm 1
\end{array}\right] \quad \text { ou } \quad\left[\begin{array}{cc} 
\pm 1 & 0 \\
\mathrm{y} & \pm 1
\end{array}\right]
$$

então $H$ contém uma transvecção.

Demonstração: Considere $\alpha=\left[\begin{array}{cc}1 & \mathrm{x} \\ 0 & 1\end{array}\right] \in H$. Como $\mathrm{x} \neq 0$, pelo menos uma de suas coordenadas é não nula. Suponha $x_{1} \neq 0$. Conjugando

$$
\begin{aligned}
& {\left[\begin{array}{cc}
0 & -\mathbf{x} \\
x_{1}^{-1} \mathbf{i} & 0
\end{array}\right]\left[\begin{array}{cc}
1 & \mathbf{x} \\
0 & 1
\end{array}\right]\left[\begin{array}{cc}
0 & \mathbf{x} \\
-\mathbf{x}_{1}^{-1} \mathbf{i} & 0
\end{array}\right]=\left[\begin{array}{cc}
0 & -\mathbf{x} \\
x_{1}^{-1} \mathbf{i} & 0
\end{array}\right]\left[\begin{array}{cc}
1 & \mathbf{x} \\
-x_{1}^{-1} \mathbf{i} & 0
\end{array}\right]} \\
& =\left[\begin{array}{cc}
1 & 0 \\
x_{1}^{-1} \mathbf{i} & 1
\end{array}\right]=\tau_{21}^{\mathbf{i}}\left(x_{1}^{-1}\right) \in H .
\end{aligned}
$$

Se o elemento $\left[\begin{array}{cc}-1 & \mathrm{x} \\ 0 & -1\end{array}\right]$ está em $H$, como $-I \in H$, então $\left[\begin{array}{cc}1 & -\mathrm{x} \\ 0 & 1\end{array}\right] \in H$ e portanto também nesse caso $H$ contém uma transvecção.

$$
\mathrm{O} \text { caso em que } \alpha=\left[\begin{array}{cc} 
\pm 1 & 0 \\
\mathrm{y} & \pm 1
\end{array}\right] \text { é análogo. }
$$

Lema 2.3.7 Se $H$ contém um elemento não central da forma

$$
\alpha=\left[\begin{array}{cc}
a & \mathrm{x} \\
0 & a^{-1}
\end{array}\right] \quad \text { ou } \quad\left[\begin{array}{cc}
a & 0 \\
\mathrm{y} & a^{-1}
\end{array}\right]
$$

com $a \neq \pm 1$. então $H$ contém uma transvecção.

Demonstração: Seja $\alpha=\left[\begin{array}{cc}a & \mathrm{x} \\ 0 & a^{-1}\end{array}\right]$, com $a \neq \pm 1$.

Consideremos primeiro o caso em que $\mathrm{x}=0$. Então $\alpha=\left[\begin{array}{cc}a & 0 \\ 0 & a^{-1}\end{array}\right]$. Conjugando $\alpha$ pela transvecção $\tau_{12}^{(\mathrm{i})}(1)$, temos

$$
\left[\begin{array}{cc}
1 & -\mathrm{i} \\
0 & 1
\end{array}\right]\left[\begin{array}{cc}
a & 0 \\
0 & a^{-1}
\end{array}\right]\left[\begin{array}{ll}
1 & \mathrm{i} \\
0 & 1
\end{array}\right]=\left[\begin{array}{cc}
a & \left(a-a^{-1}\right) \mathrm{i} \\
0 & a^{-1}
\end{array}\right]=\alpha_{1} \in H .
$$

Agora, multiplicamos $\alpha_{1}$ por $\alpha^{-1}$ :

$$
\left[\begin{array}{cc}
a & \left(a-a^{-1}\right) \mathrm{i} \\
0 & a^{-1}
\end{array}\right]\left[\begin{array}{cc}
a^{-1} & 0 \\
0 & a
\end{array}\right]=\left[\begin{array}{cc}
1 & \left(a^{2}-1\right) \mathbf{i} \\
0 & 1
\end{array}\right]=\tau_{12}^{(\mathbf{i})}\left(a^{2}-1\right) \in H .
$$


Se $\mathbf{x} \neq 0$, conjugando $\alpha$ por $\beta=\left[\begin{array}{cc}1 & \lambda \mathbf{x} \\ 0 & 1\end{array}\right]$, onde $\lambda=-\frac{1}{a-a^{-1}}$,

$$
\begin{aligned}
\beta^{-1} \alpha \beta & =\left[\begin{array}{cc}
1 & -\lambda \mathrm{x} \\
0 & 1
\end{array}\right]\left[\begin{array}{cc}
a & \mathrm{x} \\
0 & a^{-1}
\end{array}\right]\left[\begin{array}{cc}
1 & \lambda \mathrm{x} \\
0 & 1
\end{array}\right]=\left[\begin{array}{cc}
1 & -\lambda \mathrm{x} \\
0 & 1
\end{array}\right]\left[\begin{array}{cc}
a & (\lambda a+1) \mathrm{x} \\
0 & a^{-1}
\end{array}\right] \\
& =\left[\begin{array}{cc}
a & \left(\lambda a-\lambda a^{-1}+1\right) \mathrm{x} \\
0 & a^{-1}
\end{array}\right]=\left[\begin{array}{cc}
a & 0 \\
0 & a^{-1}
\end{array}\right] \in H
\end{aligned}
$$

e recaímos no caso anterior.

$\mathrm{O}$ caso em que $\alpha=\left[\begin{array}{cc}a & 0 \\ \mathrm{y} & a^{-1}\end{array}\right]$ é análogo.

Lema 2.3.8 Se $H$ contém um elemento não central da forma $\alpha=\left[\begin{array}{cc}\mp 1 & \mathrm{x} \\ \mathrm{y} & \pm 1\end{array}\right]$, então $H$ também contém uma transvecção.

Demonstração: a) Considere $\operatorname{car} K \neq 2$. Tome $\mathbf{u} \in K^{3}$ tal que $\mathbf{x} \cdot \mathbf{u}=0$.

Considere $\alpha=\left[\begin{array}{cc}1 & \mathrm{x} \\ \mathrm{y} & -1\end{array}\right]$, note que $\mathrm{x} \cdot \mathrm{y}=-2$

Conjugando

$$
\begin{aligned}
& {\left[\begin{array}{ll}
1 & 0 \\
\mathbf{u} & 1
\end{array}\right]\left[\begin{array}{cc}
1 & \mathrm{x} \\
\mathrm{y} & -1
\end{array}\right]\left[\begin{array}{cc}
1 & 0 \\
-\mathrm{u} & 1
\end{array}\right] } \\
= & {\left[\begin{array}{cc}
1 & \mathrm{x}+2 \mathrm{y} \wedge \mathrm{u} \\
\mathbf{y}+2 \mathbf{u} & -1
\end{array}\right]=\alpha_{1} \in H . }
\end{aligned}
$$

Multiplicando $\alpha$ por $\alpha_{1}$,

$$
\left[\begin{array}{cc}
1 & \mathrm{x} \\
\mathrm{y} & -1
\end{array}\right]\left[\begin{array}{cc}
1 & \mathbf{x}+2 \mathbf{y} \wedge \mathbf{u} \\
\mathbf{y}+2 \mathrm{u} & -1
\end{array}\right]=\left[\begin{array}{cc}
-1 & \mathbf{0} \\
-2 \mathrm{u} & -1
\end{array}\right] \in H
$$

Como $-I \in H$, temos que $\left[\begin{array}{cc}1 & 0 \\ 2 \mathbf{u} & 1\end{array}\right]$.

Pelo Lema 2.3.6, $H$ contém uma transvecção.

$\mathrm{O}$ caso $\left[\begin{array}{cc}-1 & \mathrm{x} \\ \mathrm{y} & 1\end{array}\right]$ é análogo, pois $-I \in H$. 
b) Considere $\operatorname{car} K=2$.

Se $\alpha=\left[\begin{array}{cc}1 & \mathrm{x} \\ \mathrm{y} & 1\end{array}\right]$, então $\mathrm{x} \cdot \mathrm{y}=0$.

Tome $\mathrm{u} \in K^{3}$ tal que $\mathbf{x} \cdot \mathrm{u}=1$. Conjugando

$$
\left[\begin{array}{cc}
1 & -0 \\
u & 1
\end{array}\right]\left[\begin{array}{cc}
1 & x \\
y & 1
\end{array}\right]\left[\begin{array}{ll}
1 & 0 \\
u & 1
\end{array}\right]=\left[\begin{array}{cc}
0 & x \\
y+u & 0
\end{array}\right]
$$

Conjugamos agora

$$
\left[\begin{array}{cc}
1 & -0 \\
y+u & 1
\end{array}\right]\left[\begin{array}{cc}
0 & x \\
y+u & 0
\end{array}\right]\left[\begin{array}{cc}
1 & 0 \\
y+u & 1
\end{array}\right]=\left[\begin{array}{ll}
1 & x \\
0 & 1
\end{array}\right]
$$

Pelo Lema 2.3.6, $H$ contém uma transvecção.

$\mathrm{O}$ caso em que $\alpha=\left[\begin{array}{cc}1 & \mathrm{x} \\ \mathrm{y} & -1\end{array}\right]$ é análogo.

Teorema 2.3.9 Seja $H \triangleleft S L L(K), Z(S L L(K)) q_{F} H$. Então $H=S L L(K)$.

Demonstração: Seja $\alpha \in H, \alpha=\left[\begin{array}{cc}a & \mathrm{x} \\ \mathrm{y} & b\end{array}\right]$ um elemento não central. Vamos mostrar que $H$ contém uma transvecção.

Suponha inicialmente que

i) $x \cdot y=0$.

Se $a \neq \pm 1$, temos $b=a^{-1}$. Considere $\beta \in S L L(K), \beta=\left[\begin{array}{cc}1 & \lambda \mathbf{x} \\ \mu \mathrm{y} & 1\end{array}\right]$, onde $\lambda=-\frac{1}{a-a^{-1}}$ e $\mu=-\frac{1}{a^{-1}-a}$. Conjugando $\beta^{-1} \alpha \beta$,

$$
\begin{aligned}
& {\left[\begin{array}{cc}
1 & -\lambda \mathbf{x} \\
-\mu \mathrm{y} & 1
\end{array}\right]\left[\begin{array}{cc}
a & \mathbf{x} \\
\mathbf{y} & a^{-1}
\end{array}\right]\left[\begin{array}{cc}
1 & \lambda \mathbf{x} \\
\mu \mathbf{y} & 1
\end{array}\right] } \\
= & {\left[\begin{array}{cc}
1 & -\lambda \mathbf{x} \\
-\mu \mathrm{y} & 1
\end{array}\right]\left[\begin{array}{cc}
a & (\lambda a+1) \mathbf{x} \\
\left(\mu a^{-1}+1\right) \mathbf{y} & a^{-1}
\end{array}\right]=\left[\begin{array}{cc}
a & 0 \\
0 & a^{-1}
\end{array}\right] \in H . }
\end{aligned}
$$

Pelo Lema 2.3.7, $H$ contém uma transvecção.

Se $a= \pm 1$. Se x ou y é nulo, o Lema 2.3.7 garante que $H$ contém uma transvecção. 
Suponhamos então $\mathrm{x} \neq 0$ e $\mathrm{y} \neq 0$ e $a=1$. Considere $\mathrm{v} \in K^{-3}$ tal que $\mathrm{x} \cdot \mathrm{v}=1$. Conjugando

$$
\alpha_{1}=\left[\begin{array}{ll}
1 & 0 \\
\mathrm{v} & 1
\end{array}\right]\left[\begin{array}{cc}
1 & \mathrm{x} \\
\mathrm{y} & 1
\end{array}\right]\left[\begin{array}{cc}
1 & 0 \\
-\mathrm{v} & 1
\end{array}\right]=\left[\begin{array}{cc}
0 & \mathrm{x}+2 \mathrm{y} \wedge \mathrm{v} \\
\mathrm{y}-\mathrm{v} & 2
\end{array}\right]
$$

Calculamos agora

$$
\alpha_{2}=\left[\begin{array}{cc}
0 & \mathrm{x}+2 \mathrm{y} \wedge \mathrm{v} \\
\mathrm{y}-\mathrm{v} & 2
\end{array}\right]\left[\begin{array}{cc}
1 & \mathrm{x} \\
\mathrm{y} & 1
\end{array}\right]=\left[\begin{array}{cc}
0 & \mathrm{x}+\mathrm{y} \wedge \mathrm{v} \\
3 \mathrm{y}-\mathrm{v} & 1
\end{array}\right] .
$$

Conjugando

$$
\begin{aligned}
\alpha_{3} & =\left[\begin{array}{cc}
0 & \mathrm{x}+\mathrm{y} \wedge \mathrm{v} \\
3 \mathrm{y}-\mathrm{v} & 0
\end{array}\right]\left[\begin{array}{cc}
0 & \mathrm{x}+\mathrm{y} \wedge \mathrm{v} \\
3 \mathrm{y}-\mathrm{v} & 1
\end{array}\right]\left[\begin{array}{cc}
0 & -\mathrm{x}-\mathrm{y} \wedge \mathrm{v} \\
-3 \mathrm{y}+\mathrm{v} & 0
\end{array}\right] \\
& =\left[\begin{array}{cc}
1 & \mathrm{x}+\mathrm{y} \wedge \mathrm{v} \\
3 \mathrm{y}-\mathrm{v} & 0
\end{array}\right] .
\end{aligned}
$$

Procedendo de maneira análoga, com um vetor $\mathrm{u} \in K^{3}$, tal que y.u $=1$, encontramos em $H$ um elemento

$$
\beta_{3}=\left[\begin{array}{cc}
0 & \mathrm{x}+\mathrm{y} \wedge \mathrm{v} \\
3 \mathrm{y}-\mathrm{v} & 1
\end{array}\right]
$$

Multiplicando $\alpha_{3}$ por $\beta_{3}$, obtemos

$$
\left[\begin{array}{cc}
1 & \mathrm{x}+\mathrm{y} \wedge \mathrm{v} \\
3 \mathrm{y}-\mathrm{v} & 0
\end{array}\right]\left[\begin{array}{cc}
0 & \mathrm{x}+\mathrm{y} \wedge \mathrm{v} \\
3 \mathrm{y}-\mathrm{v} & 1
\end{array}\right]=\left[\begin{array}{cc}
-1 & 2(\mathrm{x}+\mathrm{y} \wedge \mathrm{v}) \\
\mathbf{0} & -1
\end{array}\right]
$$

cujo quadrado é um elemento da forma $\left[\begin{array}{ll}1 & \mathrm{w} \\ 0 & 1\end{array}\right], \operatorname{com} \mathrm{w} \neq 0$ se $\operatorname{car} K \neq 2$. Pelo Lema 2.3.6, $H$ contém uma transvecção. Se car $\Lambda^{-}=2$, então $\alpha_{1}=\left[\begin{array}{cc}0 & \mathbf{x} \\ \mathrm{y}-\mathrm{v} & 0\end{array}\right]$. Conjugamos

$$
\left[\begin{array}{cc}
1 & 0 \\
\mathrm{y}-\mathrm{v} & 1
\end{array}\right]\left[\begin{array}{cc}
0 & \mathrm{x} \\
\mathrm{y}-\mathrm{v} & 0
\end{array}\right]\left[\begin{array}{cc}
1 & 0 \\
\mathrm{y}-\mathrm{v} & 1
\end{array}\right]=\left[\begin{array}{cc}
-1 & \mathrm{x} \\
0 & -1
\end{array}\right]=\left[\begin{array}{cc}
1 & \mathrm{x} \\
0 & 1
\end{array}\right] .
$$

Pelo Lema 2.3.6, $H$ contém uma transvecçào. $\mathrm{O}$ caso $a=-1$ é análogo, pois $-I \in H$.

Consideremos agora o caso 
ii) $\mathrm{x} . \mathrm{y} \neq 0$.

Afirmamos que neste caso $H$ contém um elemento da forma $\left[\begin{array}{cc}c & \mathrm{v} \\ \mathbf{0} & c^{-1}\end{array}\right]$.

De fato, para cada $m \in K$, não nulo, existe $\lambda \in K$ tal que a matriz $\beta=\left[\begin{array}{cc}m a & \lambda \mathbf{x} \\ m \mathbf{y} & -m a\end{array}\right]$ está em $S L L\left(K^{\circ}\right)$.

Para isso, basta que $\lambda$ seja raiz da equação $-m^{2} a^{2}-\lambda m x \cdot y=1$, donde

$$
\lambda=\frac{-1-m^{2} a^{2}}{m \mathrm{x} \cdot \mathrm{y}}
$$

Considere a matriz $\gamma=\beta^{-1} \alpha \beta \alpha$. Como $\beta^{-1} \alpha \beta \in H$ e $\alpha \in H$, temos que $\gamma \in H$.

Vamos calcular $\gamma$ explicitamente. Note que $\operatorname{det} \beta=-m^{2} a^{2}-\lambda m \mathrm{x} \cdot \mathrm{y}=1$. Logo,

$$
\beta^{-1}=\left[\begin{array}{cc}
-m a & -\lambda \mathbf{x} \\
-m \mathrm{y} & m a
\end{array}\right] \text {. }
$$

Então calculamos

$$
\begin{aligned}
\beta^{-1} \alpha & =\left[\begin{array}{cc}
-m a & -\lambda \mathbf{x} \\
-m \mathbf{y} & m a
\end{array}\right]\left[\begin{array}{ll}
a & \mathbf{x} \\
\mathbf{y} & b
\end{array}\right]=\left[\begin{array}{cc}
-m a^{2}-\lambda \mathbf{x} \cdot \mathbf{y} & -(m a+\lambda b) \mathbf{x} \\
-m a \mathbf{y}+m a \mathbf{y} & m a b-m \mathbf{x} \cdot \mathbf{y}
\end{array}\right] \\
\beta^{-1} \alpha & =\left[\begin{array}{cc}
1 / m & -(m a+\lambda b) \mathbf{x} \\
0 & m
\end{array}\right] \\
\beta \alpha & =\left[\begin{array}{cc}
m a & \lambda \mathbf{x} \\
m \mathbf{y} & -m a
\end{array}\right]\left[\begin{array}{cc}
a & \mathbf{x} \\
\mathbf{y} & b
\end{array}\right]=\left[\begin{array}{cc}
m a^{2}+\lambda \mathbf{x} \cdot \mathbf{y} & (m a+\lambda b) \mathbf{x} \\
0 & m \mathbf{x} \cdot \mathbf{y}-m a b
\end{array}\right] .
\end{aligned}
$$

Note que

$$
m a^{2}+\lambda \mathrm{x} \cdot \mathrm{y}=\frac{m^{2} a^{2}+\lambda m \mathrm{x} \cdot \mathrm{y}}{m}=\frac{\operatorname{det} \beta}{m}=-\frac{1}{m} .
$$

Logo,

$$
\begin{gathered}
\left(\beta^{-1} \alpha\right)(\beta \alpha)=\left[\begin{array}{cc}
1 / m & -(m a+\lambda b) \mathbf{x} \\
0 & m
\end{array}\right]\left[\begin{array}{cc}
-1 / m & (m a+\lambda b) \mathbf{x} \\
0 & -m
\end{array}\right] \\
\gamma=\left(\beta^{-1} \alpha\right)(\beta \alpha)=\left[\begin{array}{cc}
-1 / m^{2} & \rho \mathbf{x} \\
0 & -m^{2}
\end{array}\right], \text { onde } \rho=\left(\frac{1}{m}+m\right)(m a+\lambda b) .
\end{gathered}
$$


Se $m^{2} \neq \pm 1$, então. pelo Lema 2.3.7, $H$ contém uma transvecção.

Se $m^{2}= \pm 1$, então $\rho=0$ e $\gamma= \pm I$, onde $I$ é a matriz identidade.

Portanto, há no máximo quatro valores de $m$ diferentes de zero para os quais $m^{2}=$ $\pm 1$

Se $|K|>5$, é sempre possível escolher $m \in K$ não nulo, tal que $\gamma$ seja da forma $\left[\begin{array}{cc}a & \mathbf{u} \\ \mathbf{0} & a^{-1}\end{array}\right]$ e então. pelo Lema 2.3.7, $H$ contém uma transvecção.

Vamos estudar separadamente os casos em que $|K| \leq 5$.

a) $K=F_{2}$.

Seja $\alpha \in H, \alpha=\left[\begin{array}{cc}a & \mathrm{x} \\ \mathrm{y} & b\end{array}\right]$, onde $a \neq 0$, o que implica $a=1$.

Se $b=1$, então $\mathrm{x} \cdot \mathrm{y}=0$, como demonstrado no no caso (i), $H$ contém uma transvecção.

Se $b=0$, então $\alpha=\left[\begin{array}{ll}1 & \mathbf{x} \\ \mathbf{y} & 0\end{array}\right]$.

Escolha $\mathbf{u} \in K^{3}$ tal que $\mathbf{u} \neq \mathbf{y}$ e $\mathbf{u} \cdot \mathbf{x}=1$. Conjugando

$$
\left[\begin{array}{ll}
1 & \mathrm{x} \\
\mathrm{u} & 0
\end{array}\right]\left[\begin{array}{ll}
1 & \mathrm{x} \\
\mathrm{y} & 0
\end{array}\right]\left[\begin{array}{cc}
0 & \mathrm{x} \\
\mathrm{u} & 1
\end{array}\right]=\left[\begin{array}{cc}
1 & \mathrm{x}+\mathrm{y} \wedge \mathrm{u} \\
\mathbf{u}+\mathrm{x} \wedge(\mathrm{y} \wedge \mathrm{u}) & 0
\end{array}\right]=\alpha_{1} \in H
$$

Multiplicamos agora $\alpha^{-1}$ por $\alpha_{1}$ :

$$
\left[\begin{array}{ll}
0 & \mathrm{x} \\
\mathrm{y} & 1
\end{array}\right]\left[\begin{array}{cc}
1 & \mathrm{x}+\mathrm{y} \wedge \mathrm{u} \\
\mathrm{u}+\mathrm{x} \wedge(\mathrm{y} \wedge \mathrm{u}) & 0
\end{array}\right]=\left[\begin{array}{cc}
1 & \mathrm{y} \wedge \mathrm{u} \\
\mathrm{y}+\mathrm{u} & 1
\end{array}\right]=\beta \in H
$$

Como $(\mathrm{y}+\mathrm{u}) \cdot(\mathrm{y} \wedge \mathrm{u})=0$, pelo caso (i) $H$ contém uma transvecção.

b) $K=F_{3}$.

Para $m=1$ ou $m=2$, temos $m^{2}=1$. Logo, $\rho \neq 0$, a menos que $b=-a$, ou seja, para $b \neq-a, H$ contém uma transvecção pelo Lema 2.3.i. 
Se $b=-a$, entào $\alpha$ é da forma

$$
\left[\begin{array}{cc}
1 & \mathrm{x} \\
\mathrm{y} & -1
\end{array}\right] \text { ou }\left[\begin{array}{cc}
2 & \mathrm{x} \\
\mathrm{y} & -2
\end{array}\right] .
$$

Basta considerar o caso em que $\alpha$ é da forma $\left[\begin{array}{cc}1 & \mathrm{x} \\ \mathrm{y} & -1\end{array}\right]($ pois $-2 \equiv 1(\bmod 3))$.

Seja $\alpha=\left[\begin{array}{cc}1 & \mathrm{x} \\ \mathrm{y} & -1\end{array}\right]$, pelo Lema 2.3.8, $H$ contém uma transvecção.

c) $K=F_{4}=F_{2}(\xi)$,

onde $\xi$ é raiz do polinômio $f$ irredutível em $F_{2}, f=X^{2}+X+1$,

$$
K=\{0,1, \xi, \xi+1\} .
$$

Observe que $\xi^{2}=\xi+1,(\xi+1)^{2}=\xi, 1^{2}=1,0^{2}=0$.

Logo, a equação $m^{2}+1$ tem uma raiz em $K$ e a equação $m^{2} a+b$ também tem uma raiz em $K$. Nesse caso, é sempre possível escolher $m \neq 0$ tal que $m^{2} \neq \pm 1$.

d) $K=F_{5}$.

Se $m=2$ ou $m=3$, então $m^{2}=-1$ e temos $\rho=0$. Para $m=1$ ou $m=4$, temos $m^{2}=1$. Mas $\rho \neq 0$ a menos que $b=-a$.

Se $b=-a$, então $\alpha$ tem uma das seguintes formas:

$$
\left[\begin{array}{cc}
1 & \mathrm{x} \\
\mathrm{y} & -1
\end{array}\right],\left[\begin{array}{cc}
-1 & \mathrm{x} \\
\mathrm{y} & 1
\end{array}\right],\left[\begin{array}{cc}
2 & \mathrm{x} \\
\mathrm{y} & -2
\end{array}\right],\left[\begin{array}{cc}
-2 & \mathrm{x} \\
\mathrm{y} & 2
\end{array}\right]
$$

Se $\alpha$ é da forma $\left[\begin{array}{cc}2 & \mathrm{x} \\ \mathrm{y} & -2\end{array}\right]$ ou $\left[\begin{array}{cc}-2 & \mathrm{x} \\ \mathrm{y} & 2\end{array}\right]$, temos que $\mathrm{x} \cdot \mathrm{y}=0$ e caímos no caso (i).

Se $\alpha$ é da forma $\left[\begin{array}{cc}1 & \mathrm{x} \\ \mathrm{y} & -1\end{array}\right]$, então, pelo Lema 2.3.8, $H$ contém uma transvecção.

Agora vamos demonstrar o resultado principal desta seção. 
Teorema 2.3.10 PSLL( $\left.K^{\circ}\right)$ é um loop simples.

Demonstração: Seja $\Gamma$ um subloop normal em $P S L L(K)$ e considere o homomorfismo - projeção canônica

$$
w: S L L(K) \rightarrow \frac{S L L(K)}{Z_{0}}=\operatorname{PSLL}(K)
$$

Como $\Gamma \triangleleft P S L L(K)$, seja $H=w^{-1}(\Gamma)$. Então $H$ é normal em $S L L(K)$; assim:

se $H \subset Z_{0}$, então $\Gamma=(1)$ e se $H \not \subset Z_{0}$, então $H=S L L(K)$, pelo Teorema 2.3.8, donde $\Gamma=P S L L(K)$.

Podemos demonstrar ainda o seguinte

Teorema 2.3.11 $(G L L(K))^{\prime}=S L L(K)$.

Demonstração: Para simplificar a notação, vamos chamar

$$
G L L(K)=G, \quad S L L(K)=S .
$$

Observemos primeiro que para quaisquer $\alpha, \beta, \gamma$ em $G$, $[\alpha, \beta]$ e $(\alpha, \beta, \gamma)$ têm determinante 1 .

$$
\begin{aligned}
& \alpha \beta=(\beta \alpha) \cdot[\alpha, \beta] \\
& \operatorname{det} \alpha \operatorname{det} \beta=(\operatorname{det} \beta \operatorname{det} \alpha) \operatorname{det}(\alpha, \beta) \\
& \operatorname{det}(\alpha, \beta)=(\operatorname{det} \alpha)^{-1}(\operatorname{det} \beta)^{-1} \operatorname{det} \beta \operatorname{det} \alpha=1 .
\end{aligned}
$$

Analogamente,

$$
\begin{aligned}
& \alpha \beta . \gamma=(\alpha . \beta \gamma)(\alpha, \beta, \gamma) \\
& \operatorname{det}(\alpha \beta . \gamma)=\operatorname{det}(\alpha \cdot \beta \gamma) \operatorname{det}(\alpha, \beta, \gamma) \\
& \operatorname{det}(\alpha \beta) \operatorname{det} \gamma=\operatorname{det} \alpha \operatorname{det}(\beta \gamma) \operatorname{det}(\alpha, \beta \gamma) \\
& \operatorname{det}(\alpha, \beta, \gamma)=(\operatorname{det} \alpha \operatorname{det} \beta) \operatorname{det} \gamma(\operatorname{det} \alpha)^{-1}(\operatorname{det} \beta)^{-1}(\operatorname{det} \gamma)^{-1} \\
& \operatorname{det}(\alpha, \beta, \gamma)=1
\end{aligned}
$$


Assim, $G^{\prime} \subseteq S$, pois $\forall \alpha, \beta, \gamma \in G$, $\operatorname{det}(\alpha, \beta)=\operatorname{det}(\alpha, \beta, \gamma)=1$

Mostremos a inclusão contrária $S \subseteq G^{\prime}$.

Observemos que $G^{\prime} \subseteq S$ e $G^{\prime} \triangleleft G$, logo $G^{\prime} \triangleleft S$ (pois $x G^{\prime}=G^{\prime} x, \forall x \in G$ em particular para $x \in S)$.

Se car $K \neq 2$, considere a transveç̧̃̃o $\tau_{12}^{(\mathbf{i})}(\lambda)$. Mostremos que o comutador $\left[\tau_{12}^{(\mathbf{i})}(\lambda), \beta\right]$, onde $\beta=\left[\begin{array}{cc}0 & \mathrm{j} \\ -\mathrm{j} & 0\end{array}\right]$, é diferente da identidade e disto resulta que $G^{\prime}$ não está contido no centro de $S$.

$$
\begin{aligned}
{\left[\tau_{12}^{(\mathbf{i})}(\lambda), \beta\right] } & =\left[\begin{array}{cc}
1 & -\lambda \mathbf{i} \\
0 & 1
\end{array}\right]\left[\begin{array}{cc}
0 & -\mathbf{j} \\
\mathbf{j} & 0
\end{array}\right]\left[\begin{array}{cc}
1 & \lambda \mathbf{i} \\
0 & 1
\end{array}\right]\left[\begin{array}{cc}
0 & \mathbf{j} \\
-\mathbf{j} & 0
\end{array}\right] \\
& =\left[\begin{array}{cc}
0 & -\mathbf{j} \\
\mathbf{j}+\lambda \mathbf{k} & 0
\end{array}\right]\left[\begin{array}{cc}
0 & \mathbf{j} \\
-\mathbf{j}+\lambda \mathbf{k} & 0
\end{array}\right]=\left[\begin{array}{cc}
1 & 2 \lambda \mathbf{i} \\
0 & 1
\end{array}\right]=\tau_{12}^{(\mathbf{i})}(2 \lambda) \neq I,
\end{aligned}
$$

$\forall \lambda \in K$ não nulo. Donde

$$
\left[\tau_{12}^{(\mathbf{i})}(\lambda), \beta\right] \in S^{\prime} \subseteq G^{\prime} .
$$

Logo, como $G^{\prime} \triangleleft S$, pelo Teorema 2.3.9, segue que $G^{\prime}=S$.

Se car $K=2$, considere a transveç̧ão $\tau_{12}^{(\mathbf{i})}(1)$. Calculando o comutador $\left[\tau_{12}^{(\mathbf{i})}(1), \beta\right]$, onde $\beta=\left[\begin{array}{cc}0 & \mathbf{j} \\ -\mathrm{j}-\mathrm{k} & 1\end{array}\right]$, temos

$$
\begin{gathered}
{\left[\tau_{12}^{(\mathrm{i})}(1), \beta\right]=\left[\begin{array}{cc}
1 & -\mathrm{i} \\
0 & 1
\end{array}\right]\left[\begin{array}{cc}
0 & \mathrm{j} \\
-\mathrm{j}-\mathrm{k} & 1
\end{array}\right]\left[\begin{array}{cc}
1 & \mathrm{i} \\
0 & 1
\end{array}\right]\left[\begin{array}{cc}
1 & -\mathrm{j} \\
\mathrm{j}+\mathrm{k} & 0
\end{array}\right]} \\
=\left[\begin{array}{cc}
0 & \mathrm{j}-\mathrm{i} \\
-\mathrm{j}-\mathrm{k}-\mathrm{k} & 1
\end{array}\right]\left[\begin{array}{cc}
1 & -\mathrm{j} \\
\mathrm{j}+\mathrm{k}-\mathrm{k} & 0
\end{array}\right]=\left[\begin{array}{cc}
1 & 2 \mathrm{i} \\
\mathrm{k} & 1
\end{array}\right]=\left[\begin{array}{ll}
1 & 0 \\
\mathrm{k} & 1
\end{array}\right], \\
{\left[\tau_{12}^{(\mathrm{i})}(1), \beta\right] \in G^{\prime}}
\end{gathered}
$$

e pelo Teorema 2.3.9, $G^{\prime}=S$, também nesse caso.

O seguinte resultado é uma conseqüência imediata.

Corolário 2.3.12 $(S L L(K))^{\prime}=S L L(K)$. 


\subsection{As Ordens dos Loops Lineares Finitos}

No caso em que o loop inicial $K$ é finito, os loops lineares $G L L\left(K^{\circ}\right)$ e $\operatorname{PSLL}\left(K^{\circ}\right)$ são todos finitos. Nesta seção calculamos sua ordem em função da ordem de $K$.

Teorema 2.4.1 Se $K^{\prime}=F_{q}$ corpo finito com $q$ elementos, então

$$
\left|G L L\left(F_{q}\right)\right|=q^{3}\left(q^{4}-1\right)(q-1) .
$$

Demonstração: Seja $\alpha=\left[\begin{array}{cc}a & \mathrm{x} \\ \mathrm{y} & b\end{array}\right]$ um elemento de $\mathcal{Z}\left(F_{q}\right)$, então $\alpha \in G L L\left(F_{q}\right)$ se e só se $a b-\mathrm{x} \cdot \mathrm{y} \neq 0$.

Vamos então verificar quando $a b-\mathrm{x} \cdot \mathrm{y}=0$ e logo o número de elementos de $\mathcal{Z}\left(F_{q}\right)$ que não estão em $G L L\left(F_{q}\right)$. Inicialmente, vejamos quando $\mathrm{x} \cdot \mathrm{y}=0$ e quando $\mathrm{x} \cdot \mathrm{y}=m$ para $m=1,2, \ldots, q-1$
i) $x \cdot y=0$

$$
\text { \# = soluções }
$$

$$
\begin{aligned}
& x_{1} y_{1}+x_{2} y_{2}+x_{3} y_{3}=0 \\
& -x_{1} y_{1}=x_{2} y_{2}+x_{3} y_{3} \\
& x_{1} y_{1}=0 \leadsto(2 q-1) \# \\
& x_{2} y_{2}=-x_{3} y_{3}=0 \leadsto(2 q-1)^{2} \# \\
& x_{2} y_{2}=1=-x_{3} y_{3} \sim(q-1)^{2} \# \\
& \left.\begin{array}{l}
\vdots \\
x_{2} y_{2}=q-1=-x_{3} y_{3} \leadsto(q-1)^{2} \#
\end{array}\right\}(q-1) \text { equações. }
\end{aligned}
$$

Temos então

$$
\begin{array}{ll} 
& -x_{1} y_{1}=0=x_{2} y_{2}+x_{3} y_{3} \quad \text { de } \\
& (2 q-1)\left[(2 q-1)^{2}+(q-1)(q-1)^{2}\right] \# \\
\text { ou } \quad(2 q-1)\left[(2 q-1)^{2}+(q-1)^{3}\right] \#
\end{array}
$$


Podemos ter ainda a seguinte situação:

$$
\begin{aligned}
& -x_{1} y_{1}=r=x_{2} y_{2}+x_{3} y_{3} \operatorname{com} r=1,2, \ldots, q-1, \\
& -x_{1} y_{1}=r \leadsto(q-1) \# \\
& x_{2} y_{2}=r-x_{3} y_{3} \\
& \left.\begin{array}{l}
x_{3} y_{3}=r \Rightarrow x_{2} y_{2}=0 \\
x_{3} y_{3}=0 \Rightarrow x_{2} y_{2}=r
\end{array}\right\} 2(2 q-1)(q-1) \# \\
& \left.\begin{array}{l}
x_{3} y_{3}=1 \Rightarrow x_{2} y_{2}=r-1 \leadsto(q-1)^{2} \# \\
\vdots \\
x_{3} y_{3}=q-1 \Rightarrow x_{2} y_{2}=r-(q-1) \sim(q-1)^{2} \#
\end{array}\right\} q-2 \text { equações. }
\end{aligned}
$$

Assim. para cada $r$, temos

$$
(q-1)\left[2(2 q-1)(q-1)+(q-2)(q-1)^{2}\right] \# .
$$

Como temos $q-1$ valores para $r$, ficaremos com

$$
(q-1)^{2}\left[2(2 q-1)(q-1)+(q-2)(q-1)^{2}\right] \# .
$$

Juntando (2.4.1) e (2.4.2), temos x.y $=0$ de:

$$
(2 q-1)\left[(2 q-1)^{2}+(q-1)^{3}\right]+(q-1)^{2}\left[2(2 q-1)(q-1)+(q-2)(q-1)^{2}\right] \# .
$$

ii) $\mathrm{x} \cdot \mathrm{y}=m$

$$
m \in\{1,2, \ldots, q-1\} .
$$

$$
\begin{aligned}
& x_{1} y_{1}+x_{2} y_{2}+x_{3} y_{3}=m \\
& x_{1} y_{1}=m-\underbrace{\left(x_{2} y_{2}+x_{3} y_{3}\right)}_{n} \\
& \left\{\begin{array}{c}
n=0 \Rightarrow x_{1} y_{1}=m \leadsto(q-1) \# \\
n=0, \\
x_{2} y_{2}=0=x_{3} y_{3} \leadsto(2 q-1)^{2} \# \\
x_{2} y_{2}=1=x_{3} y_{3} \leadsto(q-1)^{2} \# \\
\vdots \\
x_{2} y_{2}=q-1=x_{3} y_{3} \leadsto(q-1)^{2} \#
\end{array}\right\} q-1 \text { equações. }
\end{aligned}
$$


Para $n=0$, temos

$$
\begin{gathered}
(q-1)\left[(2 q-1)^{2}+(q-1)^{3}\right] \# \\
\left\{\begin{array}{c}
n=m \Rightarrow x_{1} y_{1}=0 \leadsto(2 q-1) \# \\
n=m, \\
x_{2} y_{2}=m-x_{3} y_{3} \\
x_{3} y_{3}=0 \Rightarrow x_{2} y_{2}=m \\
x_{3} y_{3}=m \Rightarrow x_{2} y_{2}=0
\end{array}\right\} \leadsto 2(2 q-1)(q-1) \#
\end{gathered}
$$

$x_{2} y_{2}=r-x_{3} y_{3} \leadsto(q-1)^{2} \#$ em $q-2$ valores de $r$, pois $r \in\{1,2, \ldots, m-1, m+$ $1, \ldots, q-1\}$.

Para $n=m$, temos

$$
(2 q-1)\left[2(2 q-1)(q-1)+(q-2)(q-1)^{2}\right] \#
$$

Consideremos agora $n \neq 0, m$.

$$
\begin{aligned}
& x_{1} y_{1}=m-n \leadsto(q-1) \# \quad n=1,2, \ldots, \hat{m}, \ldots, q-1 \\
& x_{2} y_{2}=m-x_{3} y_{3} \\
& x_{3} y_{3}=0 \Rightarrow x_{2} y_{2}=m \\
& x_{3} y_{3}=m \Rightarrow x_{2} y_{2}=0 \\
& x_{2} y_{2}=r-x_{3} y_{3} \leadsto(q-2)(q-1)^{2} \#
\end{aligned}
$$

Para cada $n$, temos

$$
(q-1)\left[2(2 q-1)(q-1)+(q-2)(q-1)^{2}\right] .
$$

Como são $(q-2)$ valores para $n$, temos o resultado parcial:

$$
(q-2)(q-1)\left[2(2 q-1)(q-1)+(q-2)(q-1)^{2}\right] \#
$$

Juntando as possibilidades para $n=0, m$, temos $\mathrm{x} . \mathrm{y} \neq 0$ de

$$
\begin{aligned}
& (q-1)\left[(2 q-1)^{2}+(q-1)^{3}\right]+(2 q-1)\left[2(2 q-1)(q-1)+(q-2)(q-1)^{2}\right] \\
& +(q-2)(q-1)\left[2(2 q-1)(q-1)+(q-2)(q-1)^{2}\right]
\end{aligned}
$$


iii) $a b=0 \leadsto(2 q-1) \#$

$a b=m \leadsto(q-1) \#$ para qualquer valor de $m$ em $\{1,2, \ldots, q-1\}$.

iv) Logo. $a b-\mathrm{x} \cdot \mathrm{y}=0$ se:

$$
a b=0=\mathrm{x} \cdot \mathrm{y}
$$

Juntando (2.4.3), temos

$$
(2 q-1)\left\{(2 q-1)\left[(2 q-1)^{2}+(q-1)^{3}\right]+(q-1)^{2}\left[2(2 q-1)(q-1)+(q-2)(q-1)^{2}\right]\right\}
$$

$$
\begin{gathered}
a b=m=\mathbf{x} . \mathbf{y}, \quad m \in\{1,2, \ldots, q-1\} . \\
(q-1)^{3}\left[(2 q-1)^{2}+(q-1)^{3}\right]+(2 q-1)\left[2(2 q-1)(q-1)^{3}+(q-2)(q-1)^{4}\right] \\
+(q-2)(q-1)^{3}\left[2(2 q-1)(q-1)+(q-2)(q-1)^{2}\right] .
\end{gathered}
$$

Juntando (2.4.8) e (2.4.9):

$$
\begin{aligned}
& (2 q-1)^{4}+(2 q-1)^{2}(q-1)^{3}+2(2 q-1)^{2}(q-1)^{3}+(2 q-1)(q-2)(q-1)^{4} \\
& +(2 q-1)^{2}(q-1)^{3}+(q-1)^{6}+2(2 q-1)^{2}(q-1)^{3}+(2 q-1)(q-2)(q-1)^{4} \\
& +2(2 q-1)(q-2)(q-1)^{4}+(q-2)^{2}(q-1)^{5} .
\end{aligned}
$$

Simplificando,

$$
\begin{aligned}
\Gamma= & (q-2)^{2}(q-1)^{5}+4(2 q-1)(q-2)(q-1)^{4}+(q-1)^{6} \\
& +6(2 q-1)^{2}(q-1)^{3}+(2 q-1)^{4} \\
= & (q-1)^{3}\left[(q-2)^{2}(q-1)^{2}+4(2 q-1)(q-2)(q-1)+(q-1)^{3}+6(2 q-1)^{2}\right] \\
& +(2 q-1)^{4} \\
= & q^{\top}+q^{4}-q^{3} .
\end{aligned}
$$


A álgebra $\mathcal{Z}\left(F_{q}\right)$ tem ordem $q^{8}$ e $\Gamma$ representa o número de elementos em $\mathcal{Z}\left(F_{q}\right)$ cujo determinante é zero. Logo,

$$
\left|G L L\left(F_{q}\right)\right|=q^{8}-\Gamma=q^{3}\left(q^{4}-1\right)(q-1) .
$$

Como conseqüência, temos:

Corolário 2.4.2 Se $K=F_{q}$ o corpo finito com $q$ elementos, então

$$
\begin{array}{ll} 
& \left|S L L\left(F_{q}\right)\right|=q^{3}\left(q^{4}-1\right), \\
& \left|P S L L\left(F_{q}\right)\right|=\frac{q^{3}\left(q^{4}-1\right)}{2}, \text { se } q \neq 2^{n}, \\
e \quad & \left|P S L L\left(F_{q}\right)\right|=q^{3}\left(q^{4}-1\right), \text { se } q=2^{n} .
\end{array}
$$

Demonstração: $G L L\left(F_{q}\right)$ é um loop diassociativo e $S L L\left(F_{q}\right)$ é subloop normal, logo as classes laterais (à direita) de $S L L\left(F_{q}\right)$ em $G L L\left(F_{q}\right)$ são disjuntas e vale

$$
\frac{\left|G L L\left(F_{q}\right)\right|}{\left|S L L\left(F_{q}\right)\right|}=\left[G L L\left(F_{q}\right): S L L\left(F_{q}\right)\right] .
$$

Para $\alpha \in G L L\left(F_{q}\right), \operatorname{det} \alpha=1,2, \ldots, q-1$,

$$
\alpha \in S L L\left(F_{q}\right) \Leftrightarrow \operatorname{det} \alpha=1
$$

$\mathrm{O}$ número de maneiras em que det $\alpha=m$ é o mesmo para qualquer valor de $m=$ $1,2, \ldots, q-1$; logo o número de classes laterais de $S L L\left(F_{q}\right)$ em $G L L\left(F_{q}\right)$ é $q-1$. Assim,

$$
\frac{\left|G L L\left(F_{q}\right)\right|}{\left|S L L\left(F_{q}\right)\right|}=q-1
$$

ou

$$
\left|S L L\left(F_{q}\right)\right|=\frac{q^{3}\left(q^{4}-1\right)(q-1)}{(q-1)}=q^{3}\left(q^{4}-1\right) .
$$


Para encontrar a ordem de $\operatorname{PSLL}\left(F_{q}\right)$, lembremos que $\operatorname{PSLL}\left(F_{q}\right)=S L L\left(F_{q}\right) / Z_{0}$, onde $Z_{0}=Z\left(S L L\left(F_{q}\right)\right)$.

Já vimos que $Z_{0}=\{I,-I\}$.

Quando $q=2^{n}$ para algum $n \Rightarrow Z_{0}=\{I\}$, donde $\left|P S L L\left(F_{q}\right)\right|=\left|S L L\left(F_{q}\right)\right|$.

Quando $q=p^{n}, p \neq 2$, então

$$
\left|\operatorname{PSLL}\left(F_{q}\right)\right|=\frac{q^{3}\left(q^{4}-1\right)(q-1)}{2(q-1)}=\frac{q^{3}\left(q^{4}-1\right)}{2} .
$$




\section{Capítulo 3}

\section{O Menor Loop Linear}

Neste capítulo, daremos uma demonstração da simplicidade do $P S L L(K)$ quando $K=F_{2}$ e determinaremos o reticulado de subloops deste loop.

Note que $G L L\left(F_{2}\right)=S L L\left(F_{2}\right), \quad Z_{0}=\{I\}$ e $P S L L\left(F_{2}\right)=S L L\left(F_{2}\right)$ e que neste caso, $\mathrm{y} \wedge \mathrm{v}=\mathrm{v} \wedge \mathrm{y}$, para todo $\mathrm{y}, \mathrm{v} \in F_{2}^{3}$.

Do Teorema 2.4.1, vem que a ordem de $G L L\left(F_{2}\right)$ é 120 . Mostraremos mais adiante que em $\operatorname{GL} L\left(F_{2}\right)$ não valem os Teoremas de Cauchy e de Sylow, pois $5 \mid 120$ e $G L L\left(F_{2}\right)$ não tem elementos de ordem 5 .

Para os loops de Moufang não associativos de ordem menor ou igual a 63 , O. Chein provou algumas propriedades das quais faremos uso no nosso trabalho, como por exemplo, todos são de ordem par; todos contêm $p$-subgrupos de Sylow para cada $p$ dividindo a ordem do loop, e todos satisfazem o Teorema de Lagrange.

\subsection{Elementos de $G L L\left(F_{2}\right)$ - resultados preliminares}

Começaremos calculando as ordens de elementos de $G L L\left(F_{2}\right)$.

Lema 3.1.1 Todo elemento $\alpha=\left[\begin{array}{cc}a & \mathrm{x} \\ \mathrm{y} & b\end{array}\right] \in G L L\left(F_{2}\right)$ tem ordem 2 ou 3; mais precisamente, se $a=b$, então o $(\alpha)=2$ e se $a \neq b$ então $o(\alpha)=3$.

Demonstração: De fato, seja $\alpha=\left[\begin{array}{cc}a & \mathrm{x} \\ \mathrm{y} & b\end{array}\right]$. 
(i) Se $a=b$, então $\alpha$ é da forma $\alpha_{1}=\left[\begin{array}{ll}0 & \mathrm{x} \\ \mathrm{y} & 0\end{array}\right]$ ou $\alpha_{2}=\left[\begin{array}{cc}1 & \mathrm{x} \\ \mathrm{y} & 1\end{array}\right]$.

Note que $\alpha_{1}^{2}=\left[\begin{array}{cc}\mathrm{x} . \mathrm{y} & 0 \\ 0 & \mathrm{x} . \mathrm{y}\end{array}\right]$ e $\operatorname{como} \operatorname{det}\left(\alpha_{1}\right)=\mathrm{x} \cdot \mathrm{y}=1$ temos que $\alpha_{1}^{2}=I$. Também temos que $\alpha_{2}^{2}=\left[\begin{array}{cc}1+\mathrm{x} \cdot \mathrm{y} & \mathbf{0} \\ 0 & 1+\mathrm{x} . \mathrm{y}\end{array}\right]$ e, neste caso, $\operatorname{como} \operatorname{det}\left(\alpha_{2}\right)=1-\mathrm{x} \cdot \mathrm{y}=1$, temos x.y $=0$ e $\alpha_{2}^{2}=I$.

(ii) Se $a \neq b$, então $\alpha$ é da forma $\alpha_{1}=\left[\begin{array}{ll}1 & \mathrm{x} \\ \mathrm{y} & 0\end{array}\right]$ ou $\alpha_{2}=\left[\begin{array}{ll}0 & \mathrm{x} \\ \mathrm{y} & 1\end{array}\right]$. Calculamos $\alpha_{1}^{3}=\left[\begin{array}{cc}1 & 0 \\ 0 & \mathrm{x} \cdot \mathrm{y}\end{array}\right]$ e, mais uma vez, temos que $\operatorname{det}\left(\alpha_{1}\right)=\mathrm{x} \cdot \mathrm{y}$, donde $\alpha_{1}^{3}=I$.

De modo análogo, pode-se provar que $\alpha_{2}^{3}=I$.

Lema 3.1.2 Se $H$ é subloop próprio de $G L L\left(F_{2}\right)$, então $|H| \leq 60$.

Demonstração: Seja $x \in G L L\left(F_{2}\right)$ e $x \notin H$. Mostremos inicialmente que $|H|=|x H|$.

Afirmamos que $H \cap x H=\phi$.

Caso contrário, se existe $y \in H \cap x H$, então $y$ é da forma $h_{1}=x h_{2}$, com $h_{1}, h_{2} \in H$. Então temos que

$$
\begin{aligned}
& h_{1} h_{2}^{-1}=\left(x h_{2}\right) h_{2}^{-1} \in\left\langle x, h_{2}\right\rangle \quad\left(\left\langle x, h_{2}\right\rangle \text { é grupo }\right) \\
& h_{1} h_{2}^{-1}=x\left(h_{2} h_{2}^{-1}\right) \Rightarrow x=h_{1} h_{2}^{-1} \in H,
\end{aligned}
$$

o que é uma contradição.

A funçào $\mathcal{L}_{x}: H \rightarrow x H$, dada por $\mathcal{L}_{x}(h)=x h$, para todo $h \in H$, é bijetora pois é injetora (pela definição de loops) e sobrejetora.

Como $\left|G L L\left(F_{2}\right)\right|=120$, temos $|H| \leq 60$.

Definição 3.1.3 Seja $\alpha \in G L L\left(F_{2}\right)$. Definimos o centralizador de $\alpha$ e denotamos por $\mathcal{C}(\alpha)$ como sendo o conjunto

$$
\left\{\beta \in G L L\left(F_{2}\right) \mid \alpha \beta=\beta \alpha\right\}
$$


Lema 3.1.4 Seja $\alpha \in G L L\left(F_{2}\right)$. Se o $(\alpha)=3$, então $\mathcal{C}(\alpha)=\langle\alpha\rangle$.

Demonstração: Se $o(\alpha)=3$, então $\langle\alpha\rangle=\left\{1, \alpha, \alpha^{2}\right\}$ e, ainda, $\alpha$ é da forma $\left[\begin{array}{cc}0 & \mathrm{x} \\ \mathrm{y} & 1\end{array}\right]$ ou $\left[\begin{array}{ll}1 & \mathrm{x} \\ \mathrm{y} & 0\end{array}\right]$

Vamos considerar $\alpha$ da forma $\left[\begin{array}{cc}0 & \mathrm{x} \\ \mathrm{y} & 1\end{array}\right]$ e determinar os elementos de $\mathcal{C}(\alpha)$.

Note que $\alpha^{2}=\left[\begin{array}{ll}1 & \mathrm{x} \\ \mathrm{y} & 0\end{array}\right] \in\langle\alpha\rangle$.

Seja $\beta=\left[\begin{array}{ll}a & \mathbf{u} \\ \mathbf{v} & b\end{array}\right]$ tal que $\alpha \beta=\beta \alpha$. Calculemos

$$
\begin{aligned}
& \alpha \beta=\left[\begin{array}{ll}
0 & \mathrm{x} \\
\mathrm{y} & 1
\end{array}\right]\left[\begin{array}{ll}
a & \mathrm{u} \\
\mathrm{v} & b
\end{array}\right]=\left[\begin{array}{cc}
\mathrm{x} \cdot \mathrm{v} & b \mathrm{x}-\mathrm{y} \wedge \mathrm{v} \\
a \mathrm{y}+\mathrm{v}+\mathrm{x} \wedge \mathrm{u} & b+\mathrm{y} \cdot \mathrm{u}
\end{array}\right], \\
& \beta \alpha=\left[\begin{array}{ll}
a & \mathrm{u} \\
\mathrm{v} & b
\end{array}\right]\left[\begin{array}{ll}
0 & \mathrm{x} \\
\mathrm{y} & 1
\end{array}\right]\left[\begin{array}{cc}
\mathbf{y} \cdot \mathbf{u} & a \mathrm{x}+\mathrm{u}-\mathbf{v} \wedge \mathrm{y} \\
b \mathbf{y}+\mathbf{u} \wedge \mathrm{x} & b+\mathrm{x} \cdot \mathrm{v}
\end{array}\right] .
\end{aligned}
$$

Para que $\alpha \beta=\beta \alpha$, devemos ter

$$
\begin{aligned}
& \mathrm{x} \cdot \mathrm{v}=\mathrm{y} \cdot \mathrm{u} \\
& b \mathrm{x}-\mathrm{y} \wedge \mathrm{v}=a \mathrm{x}+\mathrm{u}-\mathrm{v} \wedge \mathrm{y} \Rightarrow(b-a) \mathrm{x}=\mathrm{u}+2(\mathrm{y} \wedge \mathrm{v})=\mathrm{u} \\
& b \mathrm{y}+\mathrm{u} \wedge \mathrm{x}=a \mathrm{y}+\mathrm{v}+\mathrm{x} \wedge \mathrm{u} \Rightarrow(b-a) \mathrm{y}=\mathrm{v}+2(\mathrm{x} \wedge \mathrm{u})=\mathrm{v}
\end{aligned}
$$

donde

i) se $a=b$, então $\mathrm{u}=0$ e $\mathbf{v}=0$ e segue que $a=b=1$. Logo, $\beta=\left[\begin{array}{ll}1 & 0 \\ 0 & 1\end{array}\right]$, donde 3 é a identidade.

ii) se $a \neq b$, então $\mathrm{u}=\mathrm{x} \mathrm{e} \mathrm{v}=\mathrm{y}$. Então $\beta$ terá a forma $\left[\begin{array}{ll}1 & \mathrm{x} \\ \mathrm{y} & 0\end{array}\right]$ ou $\left[\begin{array}{ll}0 & \mathrm{x} \\ \mathrm{y} & 1\end{array}\right]$, donde $\beta \in\langle\alpha\rangle$. Logo, $\mathcal{C}(\alpha)=\langle\alpha\rangle$. 
Lema 3.1.5 Se $H$ é um subloop próprio de $G L L\left(F_{2}\right)$, então $|H|=2^{a} 3^{b}$, com $0 \leq a \leq j$ $e \leq b \leq 1$

Demonstração: Pelo Lema 3.1.1, os elementos de $H$ têm ordem 2 ou 3 e como $|H| \leq 60$. o teorema de Sylow vale em $H$, donde $|H|=2^{a} 3^{b} \leq 60$. Claramente, $0 \leq a \leq 5$. Mostraremos que $b \leq 1$.

De fato, se $|H|=2^{a} 3^{2}$, então $H$ deveria conter um $S_{3}$-subgrupo de Sylow $K$ de ordem 9. Como todo 3-elemento tem ordem 3, temos que $K=\langle\alpha\rangle \times\langle\beta\rangle$, com $o(\alpha)=o(\beta)=3$. Sendo $K$ um produto direto de dois subgrupos cíclicos de ordem 3 , temos que $\alpha$ e 3 comutam, donde, $\beta \in \mathcal{C}(\alpha)$, o que é uma contradição, pelo Lema 3.1.4.

Corolário 3.1.6 Os 3-subloop de GLL(F2) são todos cíclicos de ordem 3.

\subsection{A Simplicidade de $\operatorname{PSLL}\left(F_{2}\right)$}

Vamos usar nossas informações sobre ordens obtidas acima para provar independentemente a simplicidade de $\operatorname{PS} L L\left(F_{2}\right)$.

Lema 3.2.1 Se $H \neq(I)$ é um subloop normal em $P S L L\left(F_{2}\right)$, então $H$ contém um elemento de ordem 3.

Demonstração: Seja $\alpha \in H, \alpha \neq I$. Se $o(\alpha)=3$, o resultado está provado. Se $o(\alpha)=2$. pelo Lema 3.1.1, $\alpha$ pode ser da forma $\left[\begin{array}{ll}0 & \mathrm{x} \\ \mathrm{y} & 0\end{array}\right]$ ou $\left[\begin{array}{cc}1 & \mathrm{x} \\ \mathrm{y} & 1\end{array}\right]$.

Afirmamos que se $\alpha$ é da forma $\left[\begin{array}{cc}0 & \mathrm{x} \\ \mathrm{y} & 0\end{array}\right]$, então $H$ tem um elemento $\alpha_{1}$ da forma $\left[\begin{array}{cc}1 & x^{\prime} \\ y^{\prime} & 1\end{array}\right]$. De fato, como $x \cdot y=1$. Temos que $x \neq 0$ e $y \neq 0$.

Como $\mathrm{x} \neq 0$. uma de suas coordenadas é não nula: podemos supor, sem perda de generalidade. que $x_{1} \neq 0$, o que implica dizer que $x_{1}=1$. 
Conjugando $\alpha$ pela transvecção $\tau_{21}^{(\mathrm{i})}(1)$, temos:

$$
\left[\begin{array}{ll}
1 & 0 \\
\mathrm{i} & 1
\end{array}\right]\left[\begin{array}{ll}
0 & \mathrm{x} \\
\mathrm{y} & 0
\end{array}\right]\left[\begin{array}{ll}
1 & 0 \\
\mathrm{i} & 1
\end{array}\right]=\left[\begin{array}{cc}
\mathrm{x} . \mathrm{i} & \mathrm{x} \\
\mathrm{y}+(\mathrm{x} . \mathrm{i}) \mathrm{i} & \mathrm{x.i}
\end{array}\right]=\left[\begin{array}{cc}
1 & \mathrm{x} \\
\mathrm{y}+\mathrm{i} & 1
\end{array}\right]=\alpha_{1} \in H .
$$

Vale também a recíproca: se $H$ tem um elemento $\beta$ da forma $\left[\begin{array}{cc}1 & \mathrm{x} \\ \mathrm{y} & 1\end{array}\right]=I$. Então $H$ contém um elemento da forma $\beta_{1},\left[\begin{array}{cc}0 & \mathrm{x}^{\prime} \\ \mathrm{y}^{\prime} & 0\end{array}\right]$.

De fato, se $\beta$ não é identidade, então pelo menos uma das coordenadas de $\mathbf{x}$ (ou de $\mathbf{y}$ ) é não nula. Suponha $x_{1} \neq 0$. Conjugando pela transveç̧ão $\tau_{21}^{(\mathrm{i})}$, temos:

$$
\left[\begin{array}{ll}
1 & 0 \\
\mathrm{i} & 1
\end{array}\right]\left[\begin{array}{ll}
1 & \mathrm{x} \\
\mathrm{y} & 1
\end{array}\right]\left[\begin{array}{ll}
1 & 0 \\
\mathrm{i} & 1
\end{array}\right]=\left[\begin{array}{cc}
1+\mathrm{x} . \mathrm{i} & \mathrm{x} \\
\mathrm{y}+(\mathrm{x} . \mathrm{i}) \mathrm{i} & 1+\mathrm{x} . \mathrm{i}
\end{array}\right]=\left[\begin{array}{cc}
0 & \mathrm{x} \\
\mathrm{y}+\mathrm{i} & 0
\end{array}\right] \in H .
$$

Seja então $\alpha \in H, \alpha=\left[\begin{array}{ll}0 & \mathrm{x} \\ \mathrm{y} & 0\end{array}\right], \operatorname{com} x_{1} \neq 0$ e $\alpha_{1}=\left[\begin{array}{cc}1 & \mathrm{x} \\ \mathrm{y}+\mathrm{i} & 1\end{array}\right]$ obtido acima. Logo,

$$
\alpha \alpha_{1}=\left[\begin{array}{cc}
0 & \mathrm{x} \\
\mathrm{y} & 0
\end{array}\right]\left[\begin{array}{cc}
1 & \mathrm{x} \\
\mathrm{y}+\mathrm{i} & 1
\end{array}\right]=\left[\begin{array}{cc}
1+\mathrm{x} \cdot \mathrm{i} & \mathrm{x}+\mathrm{y} \wedge \mathrm{i} \\
\mathrm{y} & 1
\end{array}\right]=\left[\begin{array}{cc}
0 & \mathrm{x}+\mathrm{y} \wedge \mathrm{i} \\
\mathrm{y} & 1
\end{array}\right]
$$

que é um elemento de ordem 3 e está em $H$.

Lema 3.2.2 Se $\alpha, \beta \in G L L\left(F_{2}\right)$ são tais que $o(\alpha)=o(\beta)=3$, então $\alpha$ é conjugado de $\beta$ ou de $\beta^{-1}$.

Demonstração: Seja $H=\langle\alpha, \beta\rangle$. Pela diassociatividade, sabemos que $H$ é um grupo e, como sua ordem é da forma $|H|=2^{a} 3$, temos que $\langle\alpha\rangle,\langle\beta\rangle$ são subgrupos de Sylow de $H$, portanto eles são subgrupos conjugados e $\alpha$ é conjugado de $\beta$ ou de $\beta^{-1}$.

Lema 3.2.3 Se $H$ é um subloop normal próprio em PSLL $\left(F_{2}\right)$, então $H$ contém todos os elementos de ordem 3 .

Demonstração: Pelo Lema 3.2.1, $H$ contém um elemento $\alpha$ de ordem 3 e, pelo lema anterior, contém todos. 
Teorema 3.2.4 PSLL $\left(F_{2}\right)$ é simples.

Demonstração: Suponhamos por absurdo que não. Então existe um subloop normal próprio $H$ de $P S L L\left(F_{2}\right)$. Pelo Lema 3.2.3, $H$ contém todos os elementos de ordem 3.

Considere os seguintes elementos $\alpha, \beta \in H$,

$$
\alpha=\left[\begin{array}{cc}
0 & (1,1,0) \\
(1,0,0) & 1
\end{array}\right], \quad \beta=\left[\begin{array}{cc}
1 & (1,0,0) \\
(1,0,1) & 0
\end{array}\right] .
$$

Então:

$$
\alpha \beta=\left[\begin{array}{cc}
0 & (1,1,0) \\
(1,0.0) & 1
\end{array}\right]\left[\begin{array}{cc}
1 & (1,0,0) \\
(1,0,1) & 0
\end{array}\right]=\left[\begin{array}{ll}
1 & \mathbf{j} \\
0 & 1
\end{array}\right]=\tau_{12}^{(\mathbf{j})}(1) \in H .
$$

Ou seja, $H$ contém uma transvecção. Pelo Lema 2.3.5, $H$ contém todas as transvecções e portanto $H=S L L\left(F_{2}\right)=G L L\left(F_{2}\right)$, o que é uma contradição.

\subsection{Limitando os Subloops de Ordem Potência de 2}

Nesta seção vamos mostrar que se $L$ é um subloop de $G L L\left(F_{2}\right)$ de ordem potência de 2 , então $|L|<16$.

Lema 3.3.1 Se o $(\alpha)=2$, então $|\mathcal{C}(\alpha)| \leq 32$.

Demonstração: Seja $\alpha=\left[\begin{array}{ll}a & \mathrm{u} \\ \mathrm{v} & a\end{array}\right]$. Considere $\beta \in \mathcal{C}(\alpha)$. Como $\alpha \in \mathcal{C}(\beta)$, se $o(\beta)=3$, segue do lema que $\alpha \in\langle\beta\rangle$, uma contradição. Então, $o(\beta)=2$ e ele é da forma $\beta=$ $\left[\begin{array}{ll}b & \mathbf{x} \\ \mathbf{y} & b\end{array}\right]$

Calculamos:

$$
\begin{aligned}
& \alpha \beta=\left[\begin{array}{ll}
a & \mathrm{u} \\
\mathrm{v} & a
\end{array}\right]\left[\begin{array}{ll}
b & \mathrm{x} \\
\mathrm{y} & b
\end{array}\right]=\left[\begin{array}{cc}
a b+\mathrm{y} \cdot \mathrm{u} & a \mathrm{x}+b \mathrm{u}+\mathrm{y} \wedge \mathrm{v} \\
a \mathbf{y}+b \mathrm{v}+\mathrm{x} \wedge \mathrm{u} & a b+\mathrm{x} \cdot \mathrm{v}
\end{array}\right] \\
& \beta \alpha=\left[\begin{array}{ll}
b & \mathrm{x} \\
\mathrm{y} & b
\end{array}\right]\left[\begin{array}{ll}
a & \mathrm{u} \\
\mathrm{v} & a
\end{array}\right]=\left[\begin{array}{cc}
a b+\mathrm{x} \cdot \mathrm{v} & a \mathrm{x}+b \mathrm{u}+\mathrm{y} \wedge \mathrm{v} \\
a \mathbf{y}+b \mathrm{v}+\mathrm{x} \wedge \mathrm{u} & a b+\mathrm{y} \cdot \mathrm{u}
\end{array}\right] .
\end{aligned}
$$

Logo, $\alpha \beta=3 \alpha$ se. e somente se, v.x = u.y.

Vamos considerar dois casos e analisar separadamente. 
i) $\mathrm{v} \cdot \mathrm{x}=\mathrm{u} \cdot \mathrm{y}=d \cdot \operatorname{com} d=0$ ou $d=1$.

Considere a equação $\mathrm{v} \cdot \mathrm{x}=d$. Como $\mathrm{v} \neq 0$, pelo menos uma de suas coordenadas é não nula, isto é, é igual a 1 . Suponhamos que $v_{1}=1$. Então, de v.x $=d$, obtemos

$$
x_{1}=d+x_{2} v_{2}+x_{3} v_{3} .
$$

Como $x_{2}$ e $x_{3}$ podem assumir os valores 0 e 1 , vemos que existem quatro vetores que são soluções desta equação. De modo análogo, existem quatro vetores que são soluções da equação $\mathrm{u} \cdot \mathrm{y}=d$. Portanto, existem 16 pares $(\mathrm{x}, \mathrm{y})$ tais que $\mathrm{v} \cdot \mathrm{x}=$ u.y $=d$, para cada valor de $d$.

Assim, há no máximo 32 pares de vetores $(\mathrm{x}, \mathrm{y})$ tais que $\mathrm{v} \cdot \mathrm{x}=\mathrm{u} \cdot \mathrm{y}$.

ii) $\mathrm{v}=0$ e $\mathrm{u} \neq 0$ (o caso $\mathrm{v} \neq 0$ e $\mathrm{u}=0$ é análogo).

Para qualquer vetor $\mathrm{x} \in F_{2}^{3}$ tem-se que $\mathrm{v} \cdot \mathrm{x}=0$. Daí, a equação $\mathrm{v} \cdot \mathrm{x}=0$ tem 8 soluções em $\mathrm{x}$ e a equação u. $\mathrm{y}=0$ tem 4 soluções em $\mathrm{y}$, como antes. Logo, existem no máximo 32 pares $(\mathbf{x}, \mathbf{y})$ que são soluções da equação $\mathbf{v} \cdot \mathbf{x}=\mathbf{u} \cdot \mathbf{y}$.

Logo, $|\mathcal{C}(\alpha)| \leq 32$.

Lema 3.3.2 Se o $(\alpha)=o(\beta)=2$, com $\alpha \neq \beta$, então $|\mathcal{C}(\alpha) \cap \mathcal{C}(\beta)| \leq 16$.

Demonstração: Sejam

$$
\alpha=\left[\begin{array}{cc}
a & \mathrm{z} \\
\mathrm{w} & a
\end{array}\right], \beta=\left[\begin{array}{cc}
b & \mathrm{u} \\
\mathbf{v} & b
\end{array}\right] \quad \text { e seja } \gamma=\left[\begin{array}{cc}
c & \mathrm{x} \\
\mathrm{y} & c
\end{array}\right]
$$

tal que $\gamma \in \mathcal{C}(\alpha) \cap \mathcal{C}(\beta)$. Para isso, é necessário que

$$
\left\{\begin{array}{l}
\text { w.x }=\text { z.y } \\
\text { v.x }=\text { u.y }
\end{array}\right.
$$

ou seja,

$$
\left\{\begin{array}{l}
w_{1} x_{1}+w_{2} x_{2}+w_{3} x_{3}=z_{1} y_{1}+z_{2} y_{2}+z_{3} y_{3} \\
v_{1} x_{1}+v_{2} x_{2}+v_{3} x_{3}=u_{1} y_{1}+u_{2} y_{2}+u_{3} y_{3}
\end{array}\right.
$$


Se $\mathbf{w}=\mathrm{v}$ e $\mathrm{z}=\mathbf{u}$. temos

$$
\alpha=\left[\begin{array}{cc}
a & \mathrm{z} \\
\mathrm{w} & a
\end{array}\right] \quad \text { e } \quad \beta=\left[\begin{array}{cc}
b & \mathrm{z} \\
\mathrm{w} & b
\end{array}\right] .
$$

Como $\operatorname{det}(\alpha)=\operatorname{det}(\beta)=1$, temos que $a^{2}+\mathrm{w} \cdot \mathrm{z}=b^{2}+\mathrm{w} \cdot \mathrm{z}$, donde $a=b$ e $\alpha=\beta$. Logo, temos que $\mathrm{w} \neq \mathrm{v}$ ou $\mathrm{z} \neq \mathrm{u}$. Se $\mathrm{w} \neq \mathrm{v}$, temos que $\mathrm{w}$ e $\mathrm{v}$ são linearmente independentes. Portanto, existe pelo menos um determinante do tipo $\left|\begin{array}{cc}w_{i} & w_{j} \\ v_{i} & v_{j}\end{array}\right| \neq 0$.

Algo análogo acontece se $\mathrm{z} \neq \mathrm{u}$. Suponhamos, para fixar idéias, que $\left|\begin{array}{cc}w_{1} & w_{2} \\ v_{1} & v_{2}\end{array}\right| \neq 0$. Consideramos o sistema

$$
\left\{\begin{array}{l}
w_{1} x_{1}+x_{2} x_{2}=w_{3} x_{3}+z_{1} y_{1}+z_{2} y_{2}+z_{3} y_{3} \\
v_{1} x_{1}+v_{2} x_{2}=v_{3} x_{3}+u_{1} y_{1}+u_{2} y_{2}+u_{3} y_{3}
\end{array}\right.
$$

Como cada uma das variáveis do segundo membro pode assumir dois valores, temos que existem $2^{4}=16$ soluçòes. Logo,

$$
|\mathcal{C}(\alpha) \cap \mathcal{C}(\beta)| \leq 16
$$

O seguinte resultado, já conhecido, será usado reiteradamente no que segue.

Lema 3.3.3 ([05]) Todo loop de Moufang de expoente 2 é um grupo e, consequentemente, é abeliano.

Corolário 3.3.4 Seja $G$ um ̊̊.-subgrupo de $G L L\left(F_{2}\right)$. Então $|G| \leq 16$.

Demonstração: Seja $G$ um 2-subgrupo e sejam $\alpha, \beta \in G, \alpha \neq \beta$. Como $G$ é abeliano, tem-se que $G \subset \mathcal{C}(\alpha) \cap \mathcal{C}(\beta)$, donde $|G| \leq|\mathcal{C}(\alpha) \cap \mathcal{C}(\beta)|=16$, pelo Lema 3.3.2,

$$
|G| \leq 16 .
$$

$$
\text { Sejam } \alpha=\left[\begin{array}{ll}
a & \mathrm{x} \\
\mathrm{y} & a
\end{array}\right], 3=\left[\begin{array}{ll}
b & \mathrm{u} \\
\mathrm{v} & b
\end{array}\right], \gamma=\left[\begin{array}{cc}
c & \mathrm{z} \\
\mathrm{w} & c
\end{array}\right] \text { três elementos de } G L L\left(F_{2}\right) \text { que }
$$
comutam dois a dois. Então temos que

$$
\left.\begin{array}{l}
\mathrm{x} \cdot \mathrm{v}=\mathrm{y} \cdot \mathrm{u} \\
\mathrm{x} \cdot \mathrm{w}=\mathrm{y} \cdot \mathrm{z} \\
\mathrm{u} \cdot \mathrm{w}=\mathrm{v} \cdot \mathrm{z}
\end{array}\right\}
$$


Vamos encontrar condições para que os três elementos associem. Para isso, calculamos

$$
\begin{aligned}
& (\alpha \beta) \gamma=\left[\begin{array}{cc}
a b+\mathrm{x} \cdot \mathrm{v} & a \mathrm{u}+b \mathbf{x}+\mathrm{y} \wedge \mathrm{v} \\
a \mathrm{v}+b \mathrm{y}+\mathrm{x} \wedge \mathrm{u} & a b+\mathrm{y} \cdot \mathbf{u}
\end{array}\right]\left[\begin{array}{cc}
c & \mathrm{z} \\
\mathrm{w} & c
\end{array}\right]=\left[\begin{array}{ll}
d_{11} & d_{12} \\
d_{21} & d_{22}
\end{array}\right] \\
& d_{11}=a b c+c \mathrm{x} \cdot \mathrm{v}+a \mathrm{u} \cdot \mathrm{w}+b \mathrm{x} \cdot \mathrm{w}+\mathrm{w} \cdot(\mathrm{y} \wedge \mathrm{v}) \\
& d_{12}=a b \mathrm{z}+b c \mathrm{x}+a c \mathrm{u}+a \mathrm{v} \wedge \mathrm{w}+b \mathrm{y} \wedge \mathrm{w}+c \mathrm{y} \wedge \mathrm{v} \\
& +(\mathrm{x} . \mathrm{v}) \mathrm{z}+(\mathrm{x} \wedge \mathrm{u}) \wedge \mathrm{w} \\
& d_{21}=a b \mathbf{w}+b c \mathbf{y}+a c \mathbf{v}+a \mathbf{u} \wedge \mathrm{z}+b \mathbf{x} \wedge \mathrm{z}+c \mathbf{x} \wedge \mathbf{u} \\
& +(\mathrm{y} \cdot \mathrm{u}) \mathrm{w}+(\mathrm{y} \wedge \mathrm{v}) \wedge \mathrm{z} \\
& d_{22}=a b c+c \mathbf{y} \cdot \mathbf{u}+a \mathbf{v} \cdot \mathbf{z}+b \mathbf{y} \cdot \mathbf{z}+\mathbf{z} \cdot(\mathbf{x} \wedge \mathbf{u})
\end{aligned}
$$

Calculamos também

$$
\alpha(\beta \gamma)=\left[\begin{array}{cc}
a & \mathrm{x} \\
\mathrm{y} & a
\end{array}\right]\left[\begin{array}{cc}
b c+\mathrm{u} \cdot \mathrm{w} & b \mathrm{z}+c \mathrm{u}+\mathrm{w} \wedge \mathrm{v} \\
b \mathrm{w}+c \mathrm{v}+(\mathrm{u} \wedge \mathrm{z}) & b c+\mathrm{v} \cdot \mathrm{z}
\end{array}\right]=\left[\begin{array}{ll}
f_{11} & f_{12} \\
f_{21} & f_{22}
\end{array}\right]
$$

onde

$$
\begin{aligned}
& f_{11}=a b c+a \mathbf{u} \cdot \mathbf{w}+b \mathrm{x} \cdot \mathbf{w}+c \mathrm{x} \cdot \mathbf{v}+\mathrm{x} \cdot(\mathbf{u} \wedge \mathrm{z}) \\
& f_{12}=a b \mathrm{z}+a c \mathbf{u}+b c \mathrm{x}+a \mathrm{v} \wedge \mathrm{w}+b \mathrm{y} \wedge \mathrm{w}+c \mathrm{y} \wedge \mathrm{v} \\
& +\mathrm{y} \wedge(\mathrm{u} \wedge \mathrm{z})+(\mathrm{v} \cdot \mathrm{z}) \mathrm{x} \\
& f_{21}=a c \mathbf{v}+a b \mathrm{w}+b c \mathrm{y}+a \mathbf{u} \wedge \mathrm{z}+b \mathrm{x} \wedge \mathrm{z}+c \mathrm{x} \wedge \mathrm{u} \\
& +\mathrm{x} \wedge(\mathrm{v} \wedge \mathrm{w})+(\mathrm{u} \cdot \mathrm{w}) \mathrm{y} \\
& f_{22}=a b c+a z \cdot v+b y \cdot z+c y \cdot u+y \cdot(v \wedge \mathrm{w})
\end{aligned}
$$

Assim, temos que

$$
\left.\begin{array}{l}
d_{11}=f_{11} \Leftrightarrow \mathrm{x} \cdot(\mathrm{u} \wedge \mathrm{z})=\mathrm{w} \cdot(\mathrm{y} \wedge \mathrm{v}) \\
d_{12}=f_{12} \Leftrightarrow(\mathrm{x} \cdot \mathrm{v}) \mathrm{z}+\mathrm{w} \wedge(\mathrm{x} \wedge \mathrm{u})=(\mathrm{v} \cdot \mathrm{z}) \mathrm{x}+\mathrm{y} \wedge(\mathrm{u} \wedge \mathrm{z}) \\
d_{21}=f_{21} \Leftrightarrow(\mathrm{y} \cdot \mathrm{u}) \mathrm{w}+\mathrm{z} \wedge(\mathrm{y} \wedge \mathrm{v})=(\mathrm{u} \cdot \mathrm{w}) \mathrm{y}+\mathrm{x} \wedge(\mathrm{v} \wedge \mathrm{w})
\end{array}\right\}
$$


Observemos que as equações (3.3.2) e (3.3.4) são equivalentes. Vamos mostrar ainda que (3.3.1) implica (3.3.3).

Lema 3.3.5 Nas condições acima, a equação (3.3.1) implica (3.3.3).

Demonstração: Calculamos

$$
\begin{aligned}
\mathbf{u} \wedge \mathbf{z}= & \left(u_{2} z_{3}-u_{3} z_{2}, u_{3} z_{1}-u_{1} z_{3}, u_{1} z_{2}-u_{2} z_{1}\right) \\
\mathbf{y} \wedge \mathbf{v}= & \left(y_{2} v_{3}-y_{3} v_{2}, y_{3} v_{1}-y_{1} v_{3}, y_{1} v_{2}-y_{2} v_{1}\right) \\
\mathbf{x} .(\mathbf{u} \wedge \mathbf{z})= & x_{1} u_{2} z_{3}-x_{1} u_{3} z_{2}+x_{2} u_{3} z_{1}-x_{2} u_{1} z_{3} \\
& +x_{3} u_{1} z_{2}-x_{3} u_{2} z_{1} \\
\mathbf{w} .(\mathbf{y} \wedge \mathbf{v})= & w_{1} y_{2} v_{3}-w_{1} y_{3} v_{2}+w_{2} y_{3} v_{1}-w_{2} y_{1} v_{3} \\
& +w_{3} y_{1} v_{2}-w_{3} y_{2} v_{1} \\
\mathbf{w} \wedge(\mathbf{x} \wedge \mathbf{u})= & \left(w_{3}\left(x_{3} u_{1}-x_{1} u_{3}\right)-w_{2}\left(x_{1} u_{2}-x_{2} u_{1}\right),\right. \\
& w_{1}\left(x_{1} u_{2}-x_{2} u_{1}\right)-w_{3}\left(x_{2} u_{3}-x_{3} u_{2}\right), w_{2}\left(x_{2} u_{3}-x_{3} u_{2}\right)-w_{1}\left(x_{3} u_{1}-x_{1} u_{3}\right) \\
\mathbf{y} \wedge(\mathbf{u} \wedge \mathbf{z})= & \left(y_{3}\left(u_{3} z_{1}-u_{1} z_{3}\right)-y_{2}\left(u_{1} z_{2}-u_{2} z_{1}\right),\right. \\
& \left.y_{1}\left(u_{1} z_{2}-u_{2} z_{1}\right)-y_{3}\left(u_{2} z_{3}-u_{3} z_{2}\right), y_{2}\left(u_{2} z_{3}-u_{3} z_{2}\right)-y_{1}\left(u_{3} z_{1}-u_{1} z_{3}\right)\right) .
\end{aligned}
$$

Vamos considerar separadamente cada coordenada de

$$
(\mathrm{x} \cdot \mathrm{v}) \mathrm{z}+\mathrm{w} \wedge(\mathrm{x} \wedge \mathrm{u})=(\mathrm{v} \cdot \mathrm{z}) \mathrm{x}+\mathrm{y} \wedge(\mathrm{u} \wedge \mathrm{z})
$$

e verificar que vale a igualdade acima se vale (3.3.1). Com efeito,

$$
\begin{aligned}
& (\mathrm{x} . \mathrm{v}) z_{1}+w_{3} x_{3} u_{1}-w_{3} x_{1} u_{3}+w_{2} x_{2} u_{1}-w_{2} x_{1} u_{2}+2 w_{1} x_{1} u_{1} \\
& =(\mathrm{v} . \mathrm{z}) x_{1}+y_{3} u_{3} z_{1}-y_{3} u_{1} z_{3}+y_{2} u_{2} z_{1}-y_{2} u_{1} z_{2}+2 y_{1} u_{1} z_{1} \\
& (\mathrm{x} . \mathrm{v}) \tilde{z}_{1}+(\mathrm{x} . \mathrm{w}) u_{1}-(\mathbf{u} \cdot \mathbf{w}) x_{1}=(\mathrm{v} . \mathrm{z}) x_{1}+(\mathrm{y} \cdot \mathrm{u}) z_{1}-(\mathrm{y} \cdot \mathbf{z}) u_{1} .
\end{aligned}
$$


Analogamente,

$$
(\mathrm{x} . \mathrm{v}) \tilde{z}_{2}+(\mathrm{x} . \mathrm{w}) u_{2}-(\mathrm{u} \cdot \mathrm{w}) x_{2}=(\mathrm{v} \cdot \mathrm{z}) x_{2}+(\mathrm{y} \cdot \mathrm{u}) \tilde{z}_{2}-(\mathrm{y} \cdot \mathrm{z}) u_{2}
$$

e também

$$
(\mathrm{x} \cdot \mathrm{v}) \tilde{z}_{3}+(\mathrm{x} \cdot \mathrm{w}) u_{3}-(\mathrm{u} \cdot \mathrm{w}) x_{3}=(\mathrm{v} \cdot \mathrm{z}) x_{3}+(\mathrm{y} \cdot \mathbf{u}) \tilde{z}_{3}-(\mathrm{y} \cdot \mathbf{z}) u_{3} .
$$

Como cada coordenada do lado direito é igual à sua correspondente do lado esquerdo, então vale a igualdade.

Procedendo de maneira análoga com a equação

$$
(\mathrm{y} \cdot \mathrm{u}) \mathrm{w}+(\mathrm{y} \wedge \mathrm{v}) \wedge \mathrm{z}=(\mathrm{u} \cdot \mathrm{w}) \mathrm{y}+\mathrm{x} \wedge(\mathrm{v} \cdot \mathrm{w})
$$

verificamos que é uma identidade. De fato,

$$
\begin{aligned}
& (\mathbf{y} . \mathbf{u}) w_{1}+z_{2} y_{1} v_{2}-z_{2} y_{2} v_{1}-z_{3} y_{3} v_{1}+z_{3} y_{1} v_{3}+2 z_{1} y_{1} v_{1} \\
& =(\mathbf{u} . \mathbf{w}) y_{1}+x_{2} v_{1} w_{2}-x_{2} v_{2} w_{1}-x_{3} v_{3} w_{1}-x_{3} v_{1} w_{3}+2 x_{1} v_{1} w_{1} \\
& (\mathbf{y . u}) w_{1}+(\mathbf{v . z}) y_{1}-(\mathbf{y} \cdot \mathbf{z}) v_{1}=(\mathbf{u} \cdot \mathbf{w}) y_{1}+(\mathbf{x} \cdot \mathbf{w}) v_{1}-(\mathrm{x} \cdot \mathbf{v}) w_{1} .
\end{aligned}
$$

Analogamente, temos

$$
(\mathbf{y} \cdot \mathbf{u}) w_{2}+(\mathbf{v} \cdot \mathbf{z}) y_{1}-(\mathbf{y} \cdot \mathbf{z}) v_{2}=(\mathbf{u} \cdot \mathbf{w}) y_{2}+(\mathbf{x} \cdot \mathbf{w}) v_{2}-(\mathbf{x} \cdot \mathbf{v}) w_{2}
$$

e também

$$
(\mathbf{y} \cdot \mathbf{u}) w_{3}+(\mathbf{v} \cdot \mathbf{z}) y_{3}-(\mathbf{y} \cdot \mathbf{z}) v_{3}=(\mathbf{u} \cdot \mathbf{w}) y_{3}+(\mathbf{x} \cdot \mathbf{w}) v_{3}+(\mathbf{x} \cdot \mathbf{v}) w_{3} .
$$

Em resumo, para que três elementos de ordem 2 comutem e associem, é necessário e suficiente que suas componentes verifiquem as equações (3.3.1) e a equação (3.3.2).

Teorema 3.3.6 $G L L\left(F_{2}\right)$ nào contém grupos de ordem 16. 
Demonstração: Suponhamos por absurdo que exista um subgrupo $G$ de ordem 16 .

Afirmação $1 G$ contém algum elemento da forma $\alpha=\left[\begin{array}{cc}a & \mathrm{u} \\ \mathrm{v} & a\end{array}\right]$ com u e v não nulos.

De fato, como existem somente 14 elementos em $G L L\left(F_{2}\right)$, onde uma das entradas vetoriais é nula e um elemento - a identidade - com as duas entradas nula, segue a afirmação.

Afirmação $2 G$ contém pelo menos dois elementos diferentes, $\alpha=\left[\begin{array}{ll}a & \mathrm{u} \\ \mathrm{v} & a\end{array}\right]$ e $\beta=$ $\left[\begin{array}{cc}b & \mathrm{z} \\ \mathbf{w} & b\end{array}\right]$, com $\mathbf{u}, \mathbf{v}, \mathbf{z}, \mathbf{w}$ não nulos.

De fato, seja $\alpha$ nas condiçòes da Afirmação 1 e seja $\gamma \in G, \gamma \neq \alpha$. Se $\gamma$ é da forma $\left[\begin{array}{ll}1 & 0 \\ \mathbf{x} & 1\end{array}\right]$, então como $\alpha \gamma=\gamma \alpha$, temos $\mathbf{u} \cdot \mathbf{x}=0$. Como $\mathbf{u} \neq 0$, existem quatro valores possíveis para $\mathbf{x}$.

De maneira análoga, podem existir no máximo quatro elementos de $G$ da forma $\left[\begin{array}{ll}1 & \mathrm{x} \\ 0 & 1\end{array}\right]$

Logo, $G$ tem, no máximo, sete elementos, onde uma das entradas vetoriais é zero.

\section{Demonstração do teorema}

Sejam $\alpha, \beta \in G$ da forma $\alpha=\left[\begin{array}{cc}a & \mathrm{u} \\ \mathrm{v} & a\end{array}\right]$ e $\beta=\left[\begin{array}{cc}b & \mathrm{z} \\ \mathrm{w} & b\end{array}\right]$, com $\mathrm{u}, \mathrm{v}, \mathrm{z}, \mathrm{w}$ não nulos.

Temos que $G \subset \mathcal{C}(\alpha) \cap \mathcal{C}(\beta)$. Mas $|G|=16 \geq|\mathcal{C}(\alpha) \cap \mathcal{C}(\beta)|$, o que quer dizer que $G=\mathcal{C}(\alpha) \cap \mathcal{C}(\beta)$.

Para obtermos uma contradição, vamos provar que existe pelo menos um elemento $\gamma \in \mathcal{C}(\alpha) \cap \mathcal{C}(\beta)$ tal que $\gamma \notin G$. Seja $\gamma=\left[\begin{array}{cc}c & \mathrm{x} \\ \mathrm{y} & c\end{array}\right]$.

Vamos considerar três casos possíveis:

1을 $\mathrm{w}=\mathrm{v}$ e $\mathrm{z} \neq \mathrm{u}$ 
Neste caso, temos que

$$
\left|\begin{array}{ccc}
y_{1} & y_{2} & y_{3} \\
w_{1} & w_{2} & w_{3} \\
v_{1} & v_{2} & v_{3}
\end{array}\right|=0, \quad \forall \mathbf{y}=\left(y_{1}, y_{2}, y_{3}\right) \in F_{2}^{3} .
$$

Se $\gamma \in G$, deve-se ter que

$$
\left\{\begin{array}{l}
\mathrm{w} \cdot \mathrm{x}=\mathrm{z} \cdot \mathrm{y} \\
\mathrm{w} \cdot \mathrm{x}=\mathrm{u} \cdot \mathrm{y}
\end{array}\right.
$$

isto é, w.x $=\mathrm{z} \cdot \mathrm{y}=\mathrm{u} \cdot \mathrm{y}$, que pode ser 0 ou 1 .

Observe que z.y = u.y = 1 implica

$$
\left\{\begin{array}{l}
z_{1} y_{1}+z_{2} y_{2}+z_{3} y_{3}=1 \\
u_{1} y_{1}+u_{2} y_{2}+u_{3} y_{3}=1
\end{array}\right.
$$

Este é um sistema de três equações com duas incógnitas e, portanto, tem solução $\mathrm{y}_{0}$.

A equação $\mathbf{w} \cdot \mathbf{x}=1 \mathrm{com} \mathbf{w} \neq 0$ tem quatro soluções, todas não nulas. Logo, existe uma solução $\mathrm{x}^{0}$ que é diferente de $0, z, \mathrm{u}$ e $\mathbf{z}+\mathrm{u}$. Como $\mathbf{z} \neq \mathbf{u}$ e três vetores diferentes de $F_{2}^{3}$ são linearmente independentes, tem-se que

$$
\left|\begin{array}{ccc}
x_{1}^{0} & x_{2}^{0} & x_{3}^{0} \\
z_{1} & z_{2} & z_{3} \\
u_{1} & u_{2} & u_{3}
\end{array}\right| \neq 0
$$

Como $\mathrm{x} \cdot(\mathbf{z} \wedge \mathrm{u}) \neq \mathrm{y} \cdot(\mathrm{w} \wedge \mathrm{v})$, tomando $\gamma_{0}=\left[\begin{array}{cc}c & \mathrm{x}_{0} \\ \mathrm{y}_{0} & c\end{array}\right]$, temos $\left(\alpha, \beta, \gamma^{0}\right) \neq 1$, donde $\gamma^{0} \notin G$.

$2^{\circ}$ caso: $\mathrm{w} \neq \mathrm{v}$ e $\mathrm{z}=\mathrm{u}$

Análogo ao anterior.

3을 caso $\neq \mathrm{v}$ e $\mathrm{z} \neq \mathrm{u}$

Queremos encontrar y tal que $\mathrm{y} \cdot(\mathrm{v} \wedge \mathrm{w})=0$, isto é, tal que

$$
\left|\begin{array}{ccc}
y_{1} & y_{2} & y_{3} \\
v_{1} & v_{2} & v_{3} \\
w_{1} & w_{2} & w_{3}
\end{array}\right|=0
$$


Existem quatro vetores tais que o determinante é nulo, a saber: $\mathrm{y}^{0}=0, \mathrm{y}^{1}=\mathrm{v}, \mathrm{y}^{2}=\mathrm{w}$, $\mathrm{y}^{3}=\mathrm{w}+\mathrm{v}$. Ainda temos que

$$
\left\{\begin{array}{l}
\mathrm{v} \cdot \mathrm{x}=\mathrm{u} \cdot \mathrm{y} \\
\mathrm{w} \cdot \mathrm{x}=\mathrm{z} \cdot \mathrm{y}
\end{array}\right.
$$

ou seja,

$$
\left\{\begin{array}{l}
v_{1} x_{1}+v_{2} x_{2}=v_{3} x_{3}+u_{1} y_{1}+u_{2} y_{2}+u_{3} y_{3} \\
w_{1} x_{1}+w_{2} x_{2}=w_{3} x_{3}+z_{1} y_{1}+z_{2} y_{2}+z_{3} y_{3}
\end{array} .\right.
$$

Como $\mathrm{v} \neq \mathrm{w}, \mathrm{v}$ e w são linearmente independentes, logo existe um menor

$$
\left|\begin{array}{cc}
v_{1} & v_{2} \\
w_{1} & w_{2}
\end{array}\right| \neq 0
$$

Assim, para cada solução $\mathrm{y}^{0}, \mathrm{y}^{1}, \mathrm{y}^{2}, \mathrm{y}^{3}$, temos duas soluções para $\mathrm{x}$, ou seja, existem oito valores para $\mathrm{x}$ satisfazendo $\left(^{*}\right)$. Portanto, existe algum $\mathrm{x} \in F_{2}^{3}$ que não anula o determinante

$$
\left|\begin{array}{lll}
x_{1} & x_{2} & x_{3} \\
u_{1} & u_{2} & u_{3} \\
z_{1} & z_{2} & z_{3}
\end{array}\right|
$$

Mas isto é equivalente a dizer que existe algum elemento $\gamma \in G L L\left(F_{2}\right)$ tal que $(\alpha, \beta, \gamma) \neq$ 1 , donde $\gamma \notin G$.

\subsection{Classificação dos 2-subloops de $G L L\left(F_{2}\right)$}

\section{Subloops de ordem 2}

Os elementos de ordem 2 em $G L L\left(F_{2}\right)$ são da forma

$$
\left[\begin{array}{ll}
0 & \mathrm{x} \\
\mathrm{y} & 0
\end{array}\right] \text { ou }\left[\begin{array}{ll}
1 & \mathrm{x} \\
\mathrm{y} & 1
\end{array}\right]
$$

Existem 28 elementos em $G L L\left(F_{2}\right)$ da forma $\left[\begin{array}{ll}0 & \mathrm{x} \\ \mathrm{y} & 0\end{array}\right]$, onde $\mathrm{x} \cdot \mathrm{y}=1$ e 36 elementos da forma $\left[\begin{array}{ll}1 & \mathrm{x} \\ \mathrm{y} & 1\end{array}\right]$ (a identidade está nesse conjunto). Portanto, são 63 elementos de ordem 2 e, consequentemente, 63 subgrupos de ordem 2.

Subloops de ordem 4 
Seja $H$ um subloop de ordem 4. Como em $G L L\left(F_{2}\right)$ não tem elemento de ordem 4, $H$ deve ser isomorfo a $C_{2} \times C_{2}$.

Dados $\alpha, \beta \in G L L\left(F_{2}\right)$, onde $o(\alpha)=o(\beta)=2, \alpha$ e $\beta$ geram um subloop de ordem 4 se $\alpha=\left[\begin{array}{ll}a & \mathrm{x} \\ \mathrm{y} & a\end{array}\right]$ e $\beta=\left[\begin{array}{ll}b & \mathrm{u} \\ \mathrm{v} & b\end{array}\right]$, então $\mathrm{x} \cdot \mathrm{v}=\mathrm{y} \cdot \mathrm{u}$.

É claro que vale a seguinte

Proposição 3.4.1 Todo subgrupo de ordem 2 está contido em pelo menos um subgrupo de ordem 4 .

\section{Subloops de ordem 8}

Seja $H$ um subloop de ordem 8 , como $H$ é um grupo, podemos ver que $H$ deve ser isomorfo a $C_{2} \times C_{2} \times C_{2}$.

Em continuação, damos um exemplo concreto de subgrupo isomorfo a $C_{2} \times C_{2} \times C_{2}$.

\section{Exemplo}

1) $\alpha=\left[\begin{array}{ll}1 & \mathrm{i} \\ \mathrm{j} & 1\end{array}\right], \quad \beta=\left[\begin{array}{ll}1 & \mathrm{j} \\ \mathrm{i} & 1\end{array}\right], \quad \gamma=\left[\begin{array}{cc}0 & \mathrm{k} \\ \mathrm{k} & 0\end{array}\right]$,

$$
\begin{aligned}
& \alpha \beta=\left[\begin{array}{cc}
0 & \mathrm{i}+\mathrm{j}+\mathrm{k} \\
\mathrm{i}+\mathrm{j}+\mathrm{k} & 0
\end{array}\right]=\beta \alpha, \quad \alpha \gamma=\left[\begin{array}{cc}
0 & \mathrm{i}+\mathrm{k} \\
\mathrm{j}+\mathrm{k} & 0
\end{array}\right]=\gamma \alpha, \\
& \beta \gamma=\left[\begin{array}{cc}
0 & \mathrm{j}+\mathrm{k} \\
\mathrm{i}+\mathrm{k} & 0
\end{array}\right]=\gamma \beta, \quad \alpha(\beta \gamma)=\left[\begin{array}{cc}
1 & \mathrm{i}+\mathrm{j} \\
\mathrm{i}+\mathrm{j} & 1
\end{array}\right]=(\alpha \beta) \gamma . \\
& \text { Portanto, } G=\langle\alpha, \beta, \gamma\rangle \cong C_{2} \times C_{2} \times C_{2} .
\end{aligned}
$$

Proposição 3.4.2 Todo subgrupo de ordem 4 está contido num subgrupo de ordem 8.

Demonstração: Seja $G=\langle\alpha, \beta\rangle \cong C_{2} \times C_{2}$, onde $\alpha=\left[\begin{array}{cc}a & \mathrm{z} \\ \mathrm{w} & a\end{array}\right], \beta=\left[\begin{array}{cc}b & \mathrm{u} \\ \mathrm{v} & b\end{array}\right]$ e tal que $\mathrm{z} \cdot \mathrm{v}=\mathrm{w} \cdot \mathrm{u}$. 
Vamos mostrar que é sempre possível encontrar $\gamma \in G L L\left(F_{2}\right), \gamma$ da forma $\left[\begin{array}{cc}c & \mathrm{x} \\ \mathrm{y} & c\end{array}\right]$. tal que $\langle\alpha, \beta, \gamma\rangle \cong C_{2} \times C_{2} \times C_{2}$. Para isso, de acordo com o Lema 3.3.5, $\gamma$ deve satisfazer

$$
\begin{aligned}
& w \cdot x=z \cdot y \\
& v \cdot x=u \cdot y \\
& x \cdot(z \wedge u)=y \cdot(w \wedge v)
\end{aligned}
$$

Ou seja, devemos verificar se o sistema abaixo tem solução em x e y:

$$
\left\{\begin{array}{l}
w_{1} x_{1}+w_{2} x_{2}+w_{3} x_{3}=z_{1} y_{1}+z_{2} y_{2}+z_{3} y_{3} \\
v_{1} x_{1}+v_{2} x_{2}+v_{3} x_{3}=u_{1} y_{1}+u_{2} y_{2}+u_{3} y_{3} \\
e_{1} x_{1}+e_{2} x_{2}+e_{3} x_{3}=f_{1} y_{1}+f_{2} y_{1}+f_{3} y_{3}
\end{array}\right.
$$

onde $\mathbf{e}=\left(e_{1}, e_{2}, e_{3}\right)=\mathbf{z} \wedge \mathbf{u}$ e $\mathbf{f}=\left(f_{1}, f_{2}, f_{3}\right)=\mathrm{w} \wedge \mathbf{v}$.

$\mathrm{O}$ sistema tem solução se o determinante abaixo é não nulo, para cada $\mathrm{y} \in F_{2}^{3}$.

$$
\left|\begin{array}{lll}
w_{1} & w_{2} & w_{3} \\
v_{1} & v_{2} & v_{3} \\
e_{1} & e_{2} & e_{3}
\end{array}\right|
$$

O determinante é nulo se o conjunto $\{w, v, e\}$ é linearmente dependente. Isto ocorre se, e somente se, se verificam as identidades

$$
\mathrm{w}=0 . \quad \mathrm{v}=0, \quad \mathrm{z} \wedge \mathrm{u}=0, \quad \mathrm{w}=\mathrm{v}, \quad \mathrm{w}=\mathrm{z} \wedge \mathrm{u}, \quad \mathrm{v}=\mathrm{z} \wedge \mathrm{u}
$$

Vamos estudar cada caso.

(i) Nos casos em que $\mathrm{w}=0, \mathrm{v}=0$ e $\mathrm{z} \wedge \mathrm{u}=0$, do sistema $\left({ }^{*}\right)$ resultam, respectivamente, os sistemas

$$
\left\{\begin{array} { l } 
{ z \cdot y = 0 } \\
{ \text { u.y =v.x } } \\
{ \text { f.y } = \text { e.x } }
\end{array} \quad \left\{\begin{array} { l } 
{ \text { w.x } = \text { z.y } } \\
{ \text { e.x } = f \cdot y } \\
{ 0 = u \cdot y }
\end{array} \quad \left\{\begin{array}{l}
\text { v.x }=\text { u.y } \\
\text { w.x }=\text { z.y } \\
0=f \cdot y
\end{array}\right.\right.\right.
$$

Todos os sistemas são de três equações com seis incógnitas. Portanto, todos têm soluçào em y. Tomando uma delas para cada caso. encontramos soluçào para $\mathrm{x} e$ nesses casos existe $\gamma$. 
(ii) Nos casos em que $\mathrm{w}=\mathrm{z} \wedge \mathrm{u}, \mathrm{v}=\mathrm{z} \wedge \mathrm{u}$ e $\mathrm{w}=\mathrm{v}$, do sistema $\left(^{*}\right.$ ) resultam, respectivamente, as equações

$$
(\mathbf{z}-\mathbf{f}) \mathbf{y}=0, \quad(\mathbf{f}-\mathbf{u}) \mathbf{y}=0, \quad(\mathbf{z}-\mathbf{u}) \mathbf{y}=0
$$

e todas têm solução em y. Tomando uma delas para cada caso, encontramos solução em x e também nesses casos, existe $\gamma$.

Como já mostramos no Teorema 3.3.6 que $G$ não contém subgrupos de ordem 16, este resultado termina a classificação dos 2-subgrupos.

\subsection{A Classificação dos Subloops de $G L L\left(F_{2}\right)$}

Já estudamos os 2-subloops de $G L L\left(F_{2}\right)$. Estudaremos agora os restantes subloops.

\section{Subloops de ordem 3}

Os elementos de ordem 3 são da forma

$$
\left[\begin{array}{cc}
1 & \mathrm{x} \\
\mathrm{y} & 0
\end{array}\right] \text { ou }\left[\begin{array}{cc}
0 & \mathrm{x} \\
\mathrm{y} & 1
\end{array}\right]
$$

onde $\mathrm{x} . \mathrm{y}=1$. Existem 28 elementos do tipo $\left[\begin{array}{ll}1 & \mathrm{x} \\ \mathrm{y} & 0\end{array}\right]$ e 28 do tipo $\left[\begin{array}{ll}0 & \mathrm{x} \\ \mathrm{y} & 1\end{array}\right]$ em $\operatorname{Gr} L\left(F_{2}\right)$, num total de 56 elementos de ordem 3. Observando que se $\alpha=\left[\begin{array}{ll}1 & \mathbf{x} \\ \mathrm{y} & 0\end{array}\right]$, então $a^{2}=$ $\left[\begin{array}{cc}0 & \mathrm{x} \\ \mathrm{y} & 1\end{array}\right]$ e. logo, temos 28 subgrupos de ordem 3 , todos conjugados entre si.

\section{Subloops de ordem 6}

Sejam a. $3 \in G L L\left(F_{2}\right), \quad \alpha=\left[\begin{array}{ll}a & \mathrm{x} \\ \mathrm{y} & a\end{array}\right], \quad \beta=\left[\begin{array}{cc}b & \mathrm{u} \\ \mathrm{v} & b\end{array}\right], \quad \alpha^{2}=\beta^{2}=1$.

Já vimos que se $\mathrm{x} . \mathrm{v}=\mathrm{y} \cdot \mathrm{u}$, entào $\alpha, \beta$ geram um subgrupo de ordem 4 . 
Consideremos então $\alpha, \beta$ tomados de tal forma que $\mathrm{x} . \mathrm{v} \neq \mathrm{y} \cdot \mathrm{u}$. O produto $\alpha \beta$ é

$$
\alpha \beta=\left[\begin{array}{cc}
a b+\mathbf{x} \cdot \mathbf{v} & a \mathbf{u}+b \mathbf{x}+\mathbf{y} \wedge \mathbf{v} \\
a \mathbf{v}+b \mathbf{y}+\mathbf{x} \wedge \mathbf{u} & a b+\mathbf{y} \cdot \mathbf{u}
\end{array}\right]
$$

donde $\alpha 3$ é um elemento de ordem 3.

$$
\beta \alpha=\left[\begin{array}{cc}
a b+\mathbf{y} \cdot \mathbf{u} & a \mathbf{u}+b \mathbf{x}+\mathbf{y} \wedge \mathbf{v} \\
a \mathbf{v}+b \mathbf{y}+\mathbf{x} \wedge \mathbf{u} & a b+\mathbf{x} \cdot \mathbf{v}
\end{array}\right] \text { nada mais é que }(\alpha \beta)^{-1}
$$

Chamando $\alpha \beta$ de $\delta$, podemos ver que

$$
\alpha \delta \alpha=\alpha(\alpha \beta) \alpha=\beta \alpha=\delta^{-1} \text {. }
$$

Assim,

$$
\left\langle\alpha, \delta \mid \alpha^{2}=\delta^{3}=1, \quad \alpha \delta \alpha=\delta^{-1}\right\rangle \cong S_{3} .
$$

De maneira geral, dado $\beta \in G L L\left(F_{2}\right)$ da forma $\left[\begin{array}{cc}c & \mathrm{x} \\ \mathrm{y} & d\end{array}\right]$, com $c \neq d$, podemos tomar $\alpha \in G L L\left(F_{2}\right)$, onde $\alpha=\left[\begin{array}{ll}a & \mathrm{x} \\ \mathrm{y} & a\end{array}\right]$, o que implica $\mathrm{x} . \mathrm{y}=1$ e $a=0$.

Suponha $c=1$ e $d=0$. Temos $\alpha^{2}=\beta^{3}=1$ e $\alpha \beta \alpha^{-1}=\beta^{-1}$. De fato,

$$
\begin{aligned}
\alpha \beta \alpha^{-1} & =\left[\begin{array}{ll}
0 & \mathrm{x} \\
\mathrm{y} & 0
\end{array}\right]\left[\begin{array}{cc}
1 & \mathrm{x} \\
\mathrm{y} & 1
\end{array}\right]\left[\begin{array}{ll}
0 & \mathrm{x} \\
\mathrm{y} & 0
\end{array}\right]=\left[\begin{array}{ll}
0 & \mathrm{x} \\
\mathrm{y} & 0
\end{array}\right]\left[\begin{array}{cc}
1 & \mathrm{x} \\
0 & 1
\end{array}\right] \\
& =\left[\begin{array}{ll}
0 & \mathrm{x} \\
\mathrm{y} & 1
\end{array}\right]=\beta^{-1},
\end{aligned}
$$

donde $\langle\alpha, \beta\rangle \cong S_{3}$.

De modo análogo, dado $\alpha$ de ordem 2 , pode-se ver que existe $\beta$ de ordem 3 tal que $\langle\alpha, \beta\rangle \cong S_{3}$. Logo, temos a seguinte

Proposição 3.5.1 Todo subgrupo de $G$ de ordem 2 ou 3 está contido num subgrupo isomorfo a $S_{3}^{\prime}$.

O seguinte lema será de utilidade adiante. 
Lema 3.5.2 Todo subloop de $G L L\left(F_{2}\right)$ isomorfo a $S_{3}$ pode ser gerado por elementos $\alpha e$ $\beta$ da forma

$$
\alpha=\left[\begin{array}{ll}
0 & \mathrm{x} \\
\mathrm{y} & 0
\end{array}\right], \quad \beta=\left[\begin{array}{ll}
1 & \mathrm{u} \\
\mathrm{v} & 0
\end{array}\right]
$$

Demonstração: Inicialmente observemos que se neste loop tem um elemento de ordem 3 , então tem um elemento $\beta$ da forma requerida (e também deve conter um elemento da forma $\left[\begin{array}{cc}0 & \mathrm{u} \\ \mathrm{v} & 1\end{array}\right]$, que é $\beta^{-1}$ ).

Se $\alpha$ é de ordem 2 e não é da forma dada no enunciado, então $\alpha=\left[\begin{array}{ll}1 & \mathrm{x} \\ \mathrm{y} & 1\end{array}\right]$, podemos encontrar $\alpha^{\prime}$ da forma $\left[\begin{array}{cc}0 & \mathrm{x}^{\prime} \\ \mathrm{y}^{\prime} & 0\end{array}\right]$. De fato, sejam $\alpha=\left[\begin{array}{cc}1 & \mathrm{x} \\ \mathrm{y} & 1\end{array}\right]$ e $\beta=\left[\begin{array}{cc}1 & \mathrm{u} \\ \mathrm{v} & 0\end{array}\right]$ tal que $\langle\alpha, \beta\rangle \cong S_{3}$. Logo, $\alpha \beta$ tem ordem 2 . Mas,

$$
\alpha \beta=\left[\begin{array}{cc}
1+\mathrm{x} \cdot \mathrm{v} & \mathrm{u}+\mathrm{y} \wedge \mathrm{v} \\
\mathrm{y}+\mathrm{v}+\mathrm{x} \wedge \mathrm{u} & \mathrm{y} \cdot \mathrm{u}
\end{array}\right] \quad \text { e } \quad \beta \alpha=\left[\begin{array}{cc}
1+\mathrm{u} \cdot \mathrm{y} & \mathrm{x}+\mathrm{u}+\mathrm{y} \wedge \mathrm{v} \\
\mathrm{v}+\mathrm{x} \wedge \mathrm{u} & \mathrm{x} \cdot \mathrm{v}
\end{array}\right] .
$$

$\alpha \beta$ tem ordem 2 se $\mathbf{x} . \mathbf{v} \neq \mathbf{y} . \mathbf{u}$.

Se $\mathrm{x} \cdot \mathbf{v}=0$ e $\mathrm{y} \cdot \mathrm{u}=1$, então $\beta \alpha=\left[\begin{array}{cc}0 & \mathrm{x}+\mathrm{u}+\mathrm{y} \wedge \mathrm{v} \\ \mathrm{v}+\mathrm{x} \wedge \mathrm{u} & 0\end{array}\right]$ e sabemos que $\langle\beta \alpha, \beta\rangle \cong S_{3}$, basta tomar $\alpha^{\prime}=\beta \alpha$.

$$
\text { Se } \mathbf{x} \cdot \mathbf{v}=1 \text { e } \mathbf{y} \cdot \mathbf{u}=0 \text {, então } \alpha \beta=\left[\begin{array}{cc}
0 & \mathbf{u}+\mathbf{y} \wedge \mathrm{v} \\
\mathbf{y}+\mathbf{v}+\mathbf{x} \wedge \mathbf{u} & 0
\end{array}\right] \text { e }\langle\alpha \beta, \beta\rangle \cong S_{3}^{\prime} \text {, basta }
$$
tomar $\alpha^{\prime}=\alpha \beta$.

Provaremos a seguir que um elemento de ordem 2 e um elemento de ordem 3 que não geram $S_{3}$ geram um subloop isomorfo a $A_{4}$.

\section{Subloops de ordem 12}

Seja $H$ um subloop de ordem 12 . $H$ pode ser um grupo ou um loop não associativo.

Se $H$ é um grupo, então $H$ deverá ser isomorfo a algum dos seguintes grupos: $C_{3} \times C_{4}$, $D_{12}, G_{12}, C_{2} \times C_{2} \times C_{3}, A_{4}$. 
$H$ nào pode ser isomorfo a $C_{3} \times C_{4}, D_{12}, G_{12}$, pois esses grupos têm elementos de ordem maior que 3 .

$H$ nào pode ser isomorfo a $C_{2} \times C_{2} \times C_{3}$, pois $H$ seria abeliano e se $\gamma \in H$ tem ordem 3, então $H \subset \mathcal{C}(\gamma)$, uma contradição.

Mostremos que em $G L L\left(F_{2}\right)$ tem subloops isomorfos a $A_{4}$.

Sejam $\alpha=\left[\begin{array}{ll}a & \mathrm{x} \\ \mathrm{y} & a\end{array}\right], \quad \beta=\left[\begin{array}{ll}1 & \mathrm{u} \\ \mathbf{v} & 0\end{array}\right], \quad$ onde $\alpha^{2}=\beta^{3}=1$. Já vimos que se $\mathbf{x} . \mathbf{v} \neq \mathbf{y} \cdot \mathbf{u}$, então $\langle\alpha, \beta\rangle \cong S_{3}$.

Se $\alpha$ e $\beta$ são tais que $\mathbf{x} \cdot \mathbf{v}=\mathrm{y} \cdot \mathbf{u}$, então $\langle\alpha, \beta\rangle \cong A_{4}$. Para isso, basta observar que $(\alpha \beta)^{3}=1 \mathrm{e}$

$$
\left\langle\alpha, \beta \mid \alpha^{2}=\beta^{3}=(\alpha \beta)^{3}=1\right\rangle \cong A_{4} .
$$

Seus elementos são:

$$
\left\{1, \alpha, \beta, \beta^{2}, \alpha \beta,(\alpha \beta)^{2}, \alpha \beta^{2},\left(\alpha \beta^{2}\right)^{2}, \alpha \beta \alpha,(\alpha \beta \alpha)^{2}, \beta^{2} \alpha \beta, \beta \alpha \beta^{2}\right\}
$$

Vamos dar um exemplo concreto de subgrupo isomorfo a $A_{4}$.

$$
\alpha=\left[\begin{array}{ll}
1 & \mathrm{i} \\
\mathrm{j} & 1
\end{array}\right], \quad \beta=\left[\begin{array}{cc}
1 & \mathrm{i}+\mathrm{j} \\
\mathrm{i} & 0
\end{array}\right] \text {. }
$$

Vamos denotar por $\mathrm{u}=\mathrm{i}+\mathrm{j}+\mathrm{k}$.

$$
\begin{array}{ll}
\beta^{2}=\left[\begin{array}{cc}
0 & \mathrm{i}+\mathrm{j} \\
\mathrm{i} & 1
\end{array}\right], & \alpha \beta=\left[\begin{array}{cc}
0 & \mathrm{u} \\
\mathrm{u} & 1
\end{array}\right], \\
(\alpha \beta)^{2}=\left[\begin{array}{cc}
1 & \mathrm{u} \\
\mathrm{u} & 0
\end{array}\right], & \alpha \beta^{2}=\left[\begin{array}{cc}
1 & \mathrm{j}+\mathrm{k} \\
\mathrm{i}+\mathrm{k} & 0
\end{array}\right], \\
\left(\alpha \beta^{2}\right)^{2}=\left[\begin{array}{cc}
0 & \mathrm{j}+\mathrm{k} \\
\mathrm{i}+\mathrm{k} & 1
\end{array}\right], & \alpha \beta \alpha=\left[\begin{array}{cc}
1 & \mathrm{j} \\
\mathrm{i}+\mathrm{j} & 0
\end{array}\right], \\
(\alpha \beta \alpha)^{2}=\left[\begin{array}{cc}
0 & \mathrm{j} \\
\mathrm{i}+\mathrm{j} & 1
\end{array}\right], & 3^{2} \alpha \beta=\left[\begin{array}{cc}
0 & \mathrm{i}+\mathrm{k} \\
\mathrm{k} & 0
\end{array}\right], \\
\beta \alpha \beta^{2}=\left[\begin{array}{cc}
0 & \mathrm{k} \\
\mathrm{j}+\mathrm{k} & 0
\end{array}\right] . &
\end{array}
$$

Como antes, temos a seguinte 
Proposição 3.5.3 Todo elemento de ordem 2 e todo elemento de ordem 3 está contido num subgrupo isomorfo a $A_{4}$.

Vamos mostrar que em $G L L\left(F_{2}\right)$ existem subloops isomorfos a $M_{12}\left(S_{3}, 2\right)$, que denotaremos brevemente por $M_{12}$. Este loop é gerado por três elementos,

$$
M_{12}=\left\langle\alpha, \beta, \gamma \mid \alpha^{2}=\beta^{3}=\gamma^{2}=1\right\rangle
$$

satisfazendo as relações

$$
\alpha^{2}=\gamma^{2}=(\alpha \beta)^{2}=(\gamma \beta)^{2}=\left(\alpha \beta^{2}\right)^{2}=\left(\gamma \beta^{2}\right)^{2}=(\alpha \gamma)^{2}=[(\alpha \gamma) \beta]^{2}=\left[(\alpha \gamma) \beta^{2}\right]^{2}=1
$$

e também

$$
\alpha \gamma=\gamma \alpha, \beta \gamma=\gamma \beta^{2}, \beta \alpha=\alpha \beta^{2}, \beta(\alpha \gamma)=(\alpha \gamma) \beta^{2}
$$

Seus elementos sào:

$$
\left\{1, \alpha, \beta, \beta^{2}, \alpha \beta, \alpha \beta^{2}, \gamma, \gamma \beta, \gamma \beta^{2}, \alpha \gamma,(\alpha \beta) \gamma, \alpha(\beta \gamma)\right\}
$$

Vamos dar um exemplo concreto de um subloop isomorfo a $M_{12}$. Seus elementos são:

$$
\begin{aligned}
& \alpha=\left[\begin{array}{ll}
1 & \mathrm{i} \\
0 & 1
\end{array}\right], \\
& \beta=\left[\begin{array}{cc}
0 & \mathrm{u} \\
\mathbf{u} & 1
\end{array}\right], \quad \gamma=\left[\begin{array}{ll}
1 & \mathrm{j} \\
0 & 1
\end{array}\right], \\
& \beta^{2}=\left[\begin{array}{ll}
1 & \mathrm{u} \\
\mathrm{u} & 0
\end{array}\right] \\
& \alpha \beta=\left[\begin{array}{cc}
1 & \mathrm{j}+\mathrm{k} \\
\mathrm{i} & 1
\end{array}\right], \quad \alpha \beta^{2}=\left[\begin{array}{cc}
0 & \mathrm{u} \\
\mathrm{i} & 0
\end{array}\right] \text {, } \\
& \alpha \gamma=\left[\begin{array}{cc}
1 & \mathrm{i}+\mathrm{j} \\
\mathrm{k} & 1
\end{array}\right]=\gamma \alpha, \quad \beta \gamma=\left[\begin{array}{ll}
0 & \mathrm{u} \\
\mathrm{j} & 0
\end{array}\right]=\gamma \beta^{2} \\
& \beta^{2} \gamma=\left[\begin{array}{cc}
1 & \mathrm{i}+\mathrm{k} \\
\mathrm{j} & 1
\end{array}\right]=\gamma \beta, \quad(\alpha \beta) \gamma=\left[\begin{array}{cc}
1 & \mathrm{k} \\
0 & 1
\end{array}\right], \quad \alpha(\beta \gamma)=\left[\begin{array}{cc}
0 & \mathrm{u} \\
\mathrm{k} & 0
\end{array}\right] \text {. }
\end{aligned}
$$

Temos então o seguinte resultado:

Proposição 3.5.4 Todo subgrupo isomorfo a $S_{3}$ está contido num subloop isomorfo a $M_{12}$. 
Demonstração: Sejam $\alpha=\left[\begin{array}{cc}0 & \mathrm{x} \\ \mathrm{y} & 0\end{array}\right], \beta=\left[\begin{array}{cc}1 & \mathrm{z} \\ \mathrm{w} & 0\end{array}\right]$ tais que $\langle\alpha, \beta\rangle \cong S_{3}$. Temos então que $\mathrm{x} \cdot \mathrm{w}=\mathrm{y} \cdot \mathrm{z}$.

Vamos mostrar que existe um elemento $\gamma=\left[\begin{array}{ll}a & \mathrm{u} \\ \mathrm{v} & a\end{array}\right] \in G L L\left(F_{2}\right)$ que satisfaz

$$
\langle\alpha, \beta, \gamma\rangle \cong M_{12}
$$

Notemos que isto implica, em particular, que $\langle\alpha, \gamma\rangle \cong\langle\beta, \gamma\rangle \cong C_{2} \times C_{2}$.

Devemos verificar que vale

$$
(\alpha \beta)^{2}=\left(\alpha 3^{2}\right)^{2}=(\gamma \beta)^{2}=\left(\gamma \beta^{2}\right)^{2}=(\alpha \gamma)^{2}=((\alpha \gamma) \beta)^{2}=\left((\alpha \gamma) \beta^{2}\right)^{2}=1
$$

e também $\beta(\alpha \gamma)=(\alpha \gamma) \beta^{2}$.

Vamos escrever os elementos

$$
\begin{aligned}
& \alpha \beta=\left[\begin{array}{cc}
\mathrm{x} \cdot \mathrm{w} & \mathrm{y} \wedge \mathrm{w} \\
\mathrm{y}+\mathrm{x} \wedge \mathrm{z} & \mathrm{y} \cdot \mathrm{z}
\end{array}\right] \quad \gamma \beta=\left[\begin{array}{cc}
a+\mathrm{u} \cdot \mathrm{w} & a \mathrm{z}+\mathrm{v} \wedge \mathrm{w} \\
\mathrm{v}+a \mathrm{w}+\mathrm{u} \wedge \mathrm{z} & \mathrm{v} \cdot \mathrm{z}
\end{array}\right] \\
& \alpha \gamma=\left[\begin{array}{cc}
\mathrm{x} \cdot \mathrm{v} & a \mathrm{x}+\mathrm{y} \wedge \mathrm{v} \\
\mathrm{x} \wedge \mathrm{u}+a \mathrm{y} & \mathrm{u} \cdot \mathrm{y}
\end{array}\right] \\
& \gamma \beta^{2}=\left[\begin{array}{cc}
\mathrm{u} . \mathrm{w} & a \mathrm{z}+\mathrm{u}+\mathrm{v} \wedge \mathrm{w} \\
a \mathrm{w}+\mathrm{u} \wedge \mathrm{z} & \mathbf{v} \cdot \mathbf{z}+a
\end{array}\right]=\beta \gamma \\
& \beta \alpha=\left[\begin{array}{cc}
\mathrm{y} \cdot \mathrm{z} & \mathrm{x}+\mathrm{y} \wedge \mathrm{w} \\
\mathrm{x} \wedge \mathrm{z} & \mathrm{x} \cdot \mathrm{w}
\end{array}\right]=\alpha \beta^{2} \\
& \beta(\alpha \gamma)=\left[\begin{array}{cc}
\mathrm{x} \cdot \mathrm{v}+a \mathrm{y} \cdot \mathrm{z}+\mathrm{z}(\mathrm{x} \wedge \mathrm{u}) & a \mathrm{x}+\mathrm{y} \wedge \mathrm{v}+(\mathrm{u} \cdot \mathrm{y}) \mathrm{z}+a(\mathrm{y} \wedge \mathrm{w})+\mathrm{w} \wedge(\mathrm{x} \wedge \mathrm{u}) \\
(\mathrm{x} \cdot \mathrm{v}) \mathrm{w}+a(\mathrm{x} \wedge \mathrm{z})+\mathrm{z} \wedge(\mathrm{y} \wedge \mathrm{v}) & a \mathrm{x} \cdot \mathrm{w}+\mathrm{w} \cdot(\mathrm{y} \wedge \mathrm{v})
\end{array}\right] \\
& (\alpha \gamma)_{i} \beta=\left[\begin{array}{cc}
\mathrm{x} \cdot \mathrm{v}+a \mathrm{x} \cdot \mathrm{w}+\mathrm{w} \cdot(\mathrm{y} \wedge \mathrm{v}) & (\mathrm{x} \cdot \mathrm{v}) \mathrm{z}+a(\mathrm{y} \wedge \mathrm{w})+\mathrm{w} \wedge(\mathrm{x} \wedge \mathrm{u}) \\
a \mathrm{y}+(\mathrm{u} \cdot \mathrm{y}) \mathrm{w}+\mathrm{x} \wedge \mathrm{u}+a \mathrm{x} \wedge \mathrm{z}+\mathrm{z} \wedge(\mathrm{y} \wedge \mathrm{v}) & a \mathrm{y} \cdot \mathrm{z}+\mathrm{z} \cdot(\mathrm{x} \wedge \mathrm{u})
\end{array}\right] \\
& (\alpha \gamma) \beta^{2}=\left[\begin{array}{cc}
a \mathrm{x} \cdot \mathrm{w}+\mathrm{w} \cdot(\mathrm{y} \wedge \mathrm{v}) & a \mathrm{x}+(\mathrm{x} \cdot \mathrm{v}) \mathrm{z}+\mathrm{y} \wedge \mathrm{v}+(\mathrm{x} \wedge \mathrm{u}) \wedge \mathrm{w}+a \mathrm{y} \wedge \mathrm{w} \\
(\mathrm{u} . \mathrm{y}) \mathrm{w}+a \mathrm{x} \wedge \mathrm{z}+(\mathrm{y} \wedge \mathrm{v}) \wedge \mathrm{z} & a \mathrm{y} \cdot \mathrm{z}+\mathrm{u} \cdot \mathrm{y}+\mathrm{z} \cdot(\mathrm{x} \wedge \mathrm{u})
\end{array}\right]
\end{aligned}
$$


Para que $(\alpha \gamma)^{2}=(\alpha \beta)^{2}=\left(\gamma \beta^{2}\right)^{2}=1$, devemos ter

$$
\left\{\begin{array}{l}
\mathrm{x} \cdot \mathrm{v}=\mathrm{y} \cdot \mathrm{u} \\
\mathrm{v} \cdot \mathrm{z}=\mathrm{u} \cdot \mathrm{w}+a
\end{array}\right.
$$

Para que $((\alpha \gamma) \beta)^{2}=\left((\alpha \gamma) \beta^{2}\right)^{2}=1$ devemos ter

$$
\mathrm{x} \cdot \mathrm{v}+a \mathrm{x} \cdot \mathrm{w}+\mathrm{w} \cdot(\mathrm{y} \wedge \mathrm{v})=a \mathrm{y} \cdot \mathrm{z}+\mathrm{z} \cdot(\mathrm{x} \wedge \mathrm{u})
$$

Para que $\beta(\alpha \gamma)=(\alpha \gamma) \beta^{2}$ devemos ter

$$
\begin{aligned}
& \mathrm{x} \cdot \mathrm{v}+a \mathrm{y} \cdot \mathrm{z}+\mathrm{z} \cdot(\mathrm{x} \wedge \mathrm{u})=a \mathrm{x} \cdot \mathrm{w}+\mathrm{w} \cdot(\mathrm{y} \wedge \mathrm{v}) \\
& a \mathrm{x} \cdot \mathrm{w}+\mathrm{w} \cdot(\mathrm{y} \wedge \mathrm{v})=a \mathrm{y} \cdot \mathrm{z}+\mathrm{u} \cdot \mathrm{y}+\mathrm{z} \cdot(\mathrm{x} \wedge \mathrm{u}) \\
& (\mathrm{u} \cdot \mathrm{y}) \mathrm{z}+\mathrm{w} \wedge(\mathrm{x} \wedge \mathrm{u})=(\mathrm{x} \cdot \mathrm{v}) \mathrm{z}+\mathrm{w} \wedge(\mathrm{x} \wedge \mathrm{u})
\end{aligned}
$$

Existe $\gamma$ nas condições requeridas se o sistema abaixo tem solução:

$$
\left\{\begin{array}{l}
\mathrm{x} \cdot \mathrm{v}=\mathrm{y} \cdot \mathrm{u} \\
\mathrm{v} \cdot \mathrm{z}=\mathrm{u} \cdot \mathrm{w}+a \\
\mathrm{x} \cdot \mathrm{v}+\mathrm{z} \cdot(\mathrm{x} \wedge \mathrm{u})=\mathrm{w} \cdot(\mathrm{y} \wedge \mathrm{v})
\end{array}\right.
$$

Vamos analisar em separado os $\operatorname{casos} \mathrm{x}=\mathrm{z}, \mathrm{y}=\mathrm{w}$ e $\mathrm{x} \neq \mathrm{z}$ ou $\mathrm{y} \neq \mathrm{w}$.

(i) $x=z$ e $y=w$.

Nesse caso, temos

$$
\left\{\begin{array}{l}
\mathrm{x} \cdot \mathrm{v}=\mathrm{y} \cdot \mathrm{u} \\
\mathrm{x} \cdot \mathrm{v}=\mathrm{y} \cdot \mathrm{u}+a \\
\mathrm{x} \cdot \mathrm{v}=0
\end{array}\right.
$$

Tomando $a=0$, teremos $\mathbf{u} \cdot \mathbf{v}=1$.

A equação $x . v=0$ tem quatro soluções. Seja $v_{0}$ uma das soluções tal que $v_{0} \neq y$ e isto implica dizer que $\mathrm{v}_{0}$ e $\mathrm{y}$ são linearmente independentes. Logo, o sistema

$$
\left\{\begin{array}{l}
\mathrm{y} \cdot \mathrm{u}=0 \\
\mathrm{v}_{0} \cdot \mathrm{u}=1
\end{array}\right.
$$

tem solução e nesse caso existe tal $\gamma$. 
(ii) $\mathrm{x} \neq \mathrm{z}$ ou $\mathrm{y} \neq \mathrm{w}$

Nesse caso, temos

$$
\left\{\begin{array}{l}
\mathrm{x} \cdot \mathrm{v}=\mathrm{y} \cdot \mathrm{u} \\
\mathrm{z} \cdot \mathrm{v}=\mathrm{w} \cdot \mathrm{u}+a \\
\mathrm{x} \cdot \mathrm{v}+(\mathrm{w} \wedge \mathrm{y}) \cdot \mathrm{v}=\mathrm{z} \cdot(\mathrm{x} \wedge \mathrm{u})
\end{array}\right.
$$

A última equação pode ser escrita como

$$
(\mathrm{x}+\mathrm{w} \wedge \mathrm{y}) \cdot \mathrm{v}=(\mathrm{z} \wedge \mathrm{x}) \cdot \mathrm{u}
$$

Se $x, z, x+w \wedge y$ são linearmente independentes, então o sistema (3.5.5) tem solução $\mathbf{u}_{0}$.

Tomando $\mathrm{u}_{0}$ encontramos $\mathrm{v}_{0}$ e nesse caso existe tal $\gamma$.

Se $\mathrm{x}, \mathrm{z}, \mathrm{x}+\mathrm{w} \wedge \mathrm{y}$ são linearmente dependentes, então

$$
\mathrm{x}+\mathrm{w} \wedge \mathrm{y}=\lambda \mathrm{x}+\mu \mathrm{z}
$$

para algum $\lambda$ e algum $\mu$.

A última equação de (3.5.5) pode ser escrita como

$$
(\mathrm{z} \wedge \mathrm{x}) \cdot \mathrm{u}=\lambda \mathrm{y} \cdot \mathrm{u}+\mu \mathrm{w} \cdot \mathrm{u}+a .
$$

que resulta em

$$
((\mathrm{z} \wedge \mathrm{x})+\lambda \mathrm{y}+\mu \mathrm{w}) \cdot \mathrm{u}=a .
$$

Nesse caso, é sempre possível encontrarmos solução em u e $a$.

Escolhendo $\mathrm{u}_{0}$ e $a_{0}$ solução de (3.5.6), temos que o sistema (3.5.5) pode ser escrito

$$
\left\{\begin{array}{l}
\mathrm{x} \cdot \mathrm{v}=\mathrm{y} \cdot \mathrm{u}_{0} \\
\mathrm{z} \cdot \mathrm{v}=\mathrm{w} \cdot \mathrm{u}_{0}+a_{0}
\end{array}\right.
$$

Esse sistema tem solução e nesse caso também existe tal $\gamma$. 


\section{Subloops de ordem 24}

Podemos ver em [15] que todos os grupos de ordem 24 têm elemento de ordem maior ou igual a 4, portanto, $G L L\left(F_{2}\right)$ não contém subgrupos de ordem 24.

Os loops de Moufang não associativos de ordem 24 descritos em [04] são: $M_{24}\left(A_{4}, 2\right)$; $M_{24}\left(D_{4}, 2\right) ; M_{24}\left(G_{12}, 2\right) ; M_{24}\left(G_{12}, C_{2} \times C_{4}\right) ; M_{24}\left(G_{12}, Q\right)$ e segundo essa mesma referência, todos estes loops têm elementos de ordem maior ou igual a 4 , exceto $M_{24}\left(A_{4}, 2\right)$, que denotaremos brevemente por $M_{24}$. Nenhum destes pode ser isomorfo a um subloop de $G L L\left(F_{2}\right)$.

Já vimos que em $G L L\left(F_{2}\right)$ existem subgrupos isomorfos a $A_{4}$, obtidos como abaixo:

$$
A_{4} \cong\left\langle\alpha, \beta \mid \alpha^{2}=\beta^{3}=(\alpha \beta)^{3}=1\right\rangle \text {. }
$$

Vamos denotar simplesmente por $L$ um destes grupos fixados.

Nosso objetivo é mostrar que todo subloop isomorfo a $A_{4}$ está contido num subloop isomorfo a $M_{24}$; para isso, bastará provar que está contido num subloop (não associativo) de ordem 24 .

Como todo elemento de ordem 2 é da forma $\left[\begin{array}{ll}1 & \mathrm{x} \\ \mathrm{y} & 1\end{array}\right]$ ou $\left[\begin{array}{ll}0 & \mathrm{x} \\ \mathrm{y} & 0\end{array}\right]$, vamos dividir a nossa demonstração em dois casos.

(I) Seja $L=\left\langle\alpha, \beta \mid \alpha^{2}=\beta^{3}=(\alpha \beta)^{3}=1\right\rangle$, onde $\alpha=\left[\begin{array}{ll}1 & \mathbf{x} \\ \mathbf{y} & 1\end{array}\right], \beta=\left[\begin{array}{ll}0 & \mathrm{u} \\ \mathbf{v} & 0\end{array}\right]$, tal que $\mathrm{x} \cdot \mathrm{v}=\mathrm{y} \cdot \mathrm{u}$.

Vamos calcular explicitamente os elementos de $L$.

$$
\begin{aligned}
& \alpha=\left[\begin{array}{ll}
1 & \mathrm{x} \\
\mathrm{y} & 1
\end{array}\right], \quad \beta=\left[\begin{array}{ll}
1 & \mathrm{u} \\
\mathrm{v} & 0
\end{array}\right], \alpha \beta^{2}=\left[\begin{array}{cc}
\mathrm{x} \cdot \mathrm{v} & \mathrm{x}+\mathrm{u}+\mathrm{y} \wedge \mathrm{v} \\
\mathrm{v}+\mathrm{x} \wedge \mathrm{u} & \mathrm{y} \cdot \mathrm{u}+1
\end{array}\right], \\
& \alpha \beta=\left[\begin{array}{cc}
1+\mathrm{x} \cdot \mathrm{v} & \mathrm{u}+\mathrm{y} \wedge \mathrm{v} \\
\mathrm{y}+\mathrm{v}+\mathrm{x} \wedge \mathrm{u} & \mathrm{y} \cdot \mathrm{u}
\end{array}\right], \\
& (\alpha \beta)^{2}=\left[\begin{array}{cc}
\mathrm{y} \cdot \mathrm{u} & \mathrm{u}+\mathrm{y} \wedge \mathrm{v} \\
\mathrm{y}+\mathrm{v}+\mathrm{x} \wedge \mathrm{u} & 1+\mathrm{x} \cdot \mathrm{v}
\end{array}\right], \quad\left(\alpha \beta^{2}\right)^{2}=\left[\begin{array}{cc}
\mathrm{y} \cdot \mathrm{u}+1 & \mathrm{x}+\mathrm{u}+\mathrm{y} \wedge \mathrm{v} \\
\mathrm{v}+\mathrm{x} \wedge \mathrm{u} & \mathrm{x} \cdot \mathrm{v}
\end{array}\right],
\end{aligned}
$$




$$
\begin{aligned}
& \alpha \beta \alpha=\left[\begin{array}{cc}
1 & (1+\mathrm{x} \cdot \mathrm{v}) \mathrm{x}+\mathrm{u}+\mathrm{y} \wedge(\mathrm{x} \wedge \mathrm{u}) \\
(1+\mathrm{y} \cdot \mathrm{u}) \mathrm{y}+\mathrm{v}+\mathrm{x} \wedge(\mathrm{y} \wedge \mathrm{v}) & 0
\end{array}\right] \\
& \beta^{2}=\left[\begin{array}{ll}
0 & \mathrm{u} \\
\mathrm{v} & 1
\end{array}\right] \\
& (\alpha \beta \alpha)^{2}=\left[\begin{array}{cc}
0 & (1+\mathrm{x} \cdot \mathrm{v}) \mathrm{x}+\mathrm{u}+\mathrm{y} \wedge(\mathrm{x} \wedge \mathrm{u}) \\
(1+\mathrm{y} \cdot \mathrm{u}) \mathrm{y}+\mathrm{v}+\mathrm{x} \wedge(\mathrm{y} \wedge \mathrm{v}) & 1
\end{array}\right] \\
& \beta^{2} \alpha \beta=\left[\begin{array}{cc}
\mathrm{y} \cdot \mathrm{u}+\mathrm{u} \cdot \mathrm{v} & (\mathrm{y} \cdot \mathrm{u}) \mathrm{u}+\mathrm{y} \wedge \mathrm{v}+\mathrm{v} \wedge(\mathrm{x} \wedge \mathrm{u}) \\
(\mathrm{x} . \mathrm{v}) \mathrm{v}+\mathrm{y}+\mathrm{x} \wedge \mathrm{u}+\mathrm{u} \wedge(\mathrm{y} \wedge \mathrm{v}) & \mathrm{u} \cdot \mathrm{v}+\mathrm{y} \cdot \mathrm{u}
\end{array}\right] \\
& \beta \alpha \beta^{2}=\left[\begin{array}{cc}
\mathrm{x} \cdot \mathrm{v}+\mathrm{u} \cdot \mathrm{v} & (\mathrm{x} \cdot \mathrm{v}) \mathrm{u}+\mathrm{x}+\mathrm{y} \wedge \mathrm{v}+\mathrm{v} \wedge(\mathrm{x} \wedge \mathrm{u}) \\
(\mathrm{y} \cdot \mathrm{u}) \mathrm{v}+\mathrm{x} \wedge \mathrm{u}+\mathrm{u} \wedge(\mathrm{y} \wedge \mathrm{v}) & \mathrm{x} \cdot \mathrm{v}+\mathrm{u} \cdot \mathrm{v}
\end{array}\right]
\end{aligned}
$$

Vamos mostrar inicialmente que é possível encontrar um elemento $\gamma \in G L L\left(F_{2}\right)$ da forma $\gamma=\left[\begin{array}{cc}0 & \mathrm{z} \\ \mathrm{w} & 0\end{array}\right], \gamma$ de tal modo que $(g \gamma)^{2}=1$, para todo $g \in L$.

Se $g=\alpha$, então para que $\alpha \gamma=\gamma \alpha$ devemos ter $\mathbf{x} \cdot \mathbf{w}=\mathbf{y} \cdot \mathbf{z}$.

Se $g=\beta$, então para que $\beta \gamma=\gamma \beta^{2}$ devemos ter $\mathrm{u} . \mathrm{w}=\mathrm{v} . \mathrm{z}$. Temos a mesma relação se $g=\beta^{2}$.

Para decidir se um elemento é de ordem 2 , basta verificar que os elementos da diagonal principal são iguais. Vamos calcular estes elementos em todos os casos possíveis.

Se $g=\left(\alpha \beta^{2}\right)^{2}$ ou $g=\left(\alpha \beta^{2}\right)$, devemos ter $\mathrm{u} \cdot \mathrm{w}+\mathrm{w} \cdot(\mathrm{y} \wedge \mathrm{v})=\mathrm{v} \cdot \mathrm{z}+\mathrm{z} \cdot(\mathbf{x} \wedge \mathrm{u})+\mathrm{y} \cdot \mathbf{z}$.

Se $g=(\alpha \beta)^{2}$ ou $g=\alpha \beta$, devemos ter $\mathbf{x} \cdot \mathbf{w}+\mathbf{u} \cdot \mathbf{w}+\mathbf{w} \cdot(\mathbf{y} \wedge \mathbf{v})=\mathbf{v} \cdot \mathbf{z}+\mathbf{z} \cdot(\mathbf{x} \wedge \mathbf{u})$.

Se $g=\beta^{2} \alpha \beta$, devemos ter

$$
(y \cdot u)(u \cdot w)+w \cdot(y \wedge v)+w \cdot(v \wedge(x \wedge u))=(x \cdot v)(v \cdot z)+y \cdot z+z \cdot(x \wedge u)+z \cdot(u \wedge(y \wedge v))
$$

Se $g=\beta \alpha \beta^{2}$, devemos ter . $(\mathrm{x} \cdot \mathrm{v})(\mathrm{u} \cdot \mathrm{w})+\mathrm{x} \cdot \mathrm{w}+\mathrm{w} \cdot(\mathrm{y} \wedge \mathrm{v})+\mathrm{w} \cdot(\mathrm{v} \wedge(\mathrm{x} \wedge \mathrm{u}))=(\mathrm{v} \cdot \mathrm{z})(\mathrm{y} \cdot \mathrm{u})+\mathrm{z} \cdot(\mathrm{x} \wedge \mathrm{u})+\mathrm{z} \cdot(\mathrm{u} \wedge(\mathrm{y} \wedge \mathrm{v}))$ 
Se $g=\alpha \beta \alpha$ ou $g=(\alpha \beta \alpha)^{2}$, devemos ter

$$
(\mathrm{x} \cdot \mathrm{w})(\mathrm{x} \cdot \mathrm{v})+\mathrm{x} \cdot \mathrm{w}+\mathrm{u} \cdot \mathrm{w}+\mathrm{w} \cdot(\mathrm{y} \wedge(\mathrm{x} \wedge \mathrm{u}))=(\mathrm{y} \cdot \mathrm{z})(\mathrm{y} \cdot \mathrm{u})+\mathrm{y} \cdot \mathrm{z}+\mathrm{v} \cdot \mathrm{z}+\mathrm{z} \cdot(\mathrm{x} \wedge(\mathrm{y} \wedge \mathrm{v}))
$$

O elemento $\gamma$ verifica a condição requerida se, e somente se, as seguintes condições são satisfeitas:

(i) $\mathrm{x} \cdot \mathrm{v}=\mathrm{y} \cdot \mathrm{u}$;

(ii) $\mathrm{x} \cdot \mathrm{w}=\mathrm{y} \cdot \mathrm{z}$;

(iii) $\mathrm{u} . \mathrm{w}=\mathrm{v} . \mathrm{z}$;

(iv) $\mathrm{x} \cdot \mathrm{w}+\mathrm{u} \cdot \mathrm{w}+\mathrm{w} \cdot(\mathrm{y} \wedge \mathrm{v})=\mathrm{v} \cdot \mathrm{z}+\mathrm{z} \cdot(\mathrm{x} \wedge \mathrm{u})$;

(v) u.w $+\mathrm{w} \cdot(\mathrm{y} \wedge \mathrm{v})=\mathrm{y} \cdot \mathrm{z}+\mathrm{v} \cdot \mathrm{z}+\mathrm{z} \cdot(\mathrm{x} \wedge \mathrm{u})$;

(vi) $(\mathrm{y} \cdot \mathrm{u})(\mathrm{u} \cdot \mathrm{w})+\mathrm{w} \cdot(\mathrm{y} \wedge \mathrm{v})+\mathrm{w} \cdot(\mathrm{v} \wedge(\mathrm{x} \wedge \mathrm{u}))=(\mathrm{x} \cdot \mathrm{v})(\mathrm{v} \cdot \mathrm{z})+\mathrm{y} \cdot \mathrm{z}+\mathrm{z} \cdot(\mathrm{x} \wedge \mathrm{u})+\mathrm{z} \cdot(\mathrm{u} \wedge(\mathrm{y} \wedge \mathrm{v}))$

(vii) $(\mathrm{x} \cdot \mathrm{v})(\mathrm{u} \cdot \mathrm{w})+\mathrm{x} \cdot \mathrm{w}+\mathrm{w} \cdot(\mathrm{y} \wedge \mathrm{v})+\mathrm{w} \cdot(\mathrm{v} \wedge(\mathrm{x} \wedge \mathrm{u}))=(\mathrm{v} \cdot \mathrm{z})(\mathrm{y} \cdot \mathrm{u})+\mathrm{z} \cdot(\mathrm{x} \wedge \mathrm{u})+\mathrm{z} \cdot(\mathrm{u} \wedge(\mathrm{y} \wedge \mathrm{v}))$

$($ viii $)(\mathrm{x} \cdot \mathrm{w})(\mathrm{x} \cdot \mathrm{v})+\mathrm{x} \cdot \mathrm{w}+\mathrm{u} \cdot \mathrm{w}+\mathrm{w} \cdot(\mathrm{y} \wedge(\mathrm{x} \wedge \mathrm{u}))=(\mathrm{y} \cdot \mathrm{z})(\mathrm{y} \cdot \mathrm{u})+\mathrm{y} \cdot \mathrm{z}+\mathrm{v} \cdot \mathrm{z}+\mathrm{z} \cdot(\mathrm{x} \wedge(\mathrm{y} \wedge \mathrm{v}))$.

As condições (ii) e (iii) mostram que as condições (iv) e (v) são equivalentes.

As condições (i) e (iii) mostram que as condições (vi) e (vii) podem ser escritas como

$$
\mathrm{y} \cdot \mathrm{z}+\mathrm{w} \cdot(\mathrm{y} \wedge \mathrm{v})+\mathrm{w} \cdot(\mathrm{v} \wedge(\mathrm{x} \wedge \mathrm{u}))=\mathrm{z} \cdot(\mathrm{x} \wedge \mathrm{u})+\mathrm{z} \cdot(\mathrm{u} \wedge(\mathrm{y} \wedge \mathrm{v}))
$$

As condições (i) e (ii) mostram que a condição (viii) pode ser escrita como

$$
\mathrm{w} \cdot(\mathrm{y} \wedge(\mathrm{x} \wedge \mathrm{u}))=\mathrm{z} \cdot(\mathrm{x} \wedge(\mathrm{y} \wedge \mathrm{v}))
$$

Portanto, o sistema acima é equivalente ao seguinte:

(i) $\mathrm{x} \cdot \mathrm{v}=\mathrm{y} \cdot \mathrm{u}$; 
(ii) $\mathrm{x} \cdot \mathrm{w}=\mathrm{y} \cdot \mathrm{z}$;

(iii) u.w = v.z;

(iv) $y \cdot z+w \cdot(y \wedge v)=z \cdot(x \wedge u)$;

(v) w. $(y \wedge(x \wedge u))=z \cdot(x \wedge(y \wedge v))$

(vi) $\mathrm{w} \cdot(\mathrm{v} \wedge(\mathrm{x} \wedge \mathrm{u}))=\mathrm{z} \cdot(\mathrm{u} \wedge(\mathrm{y} \wedge \mathrm{v}))$.

Lema 3.5.5 As condições (i), (ii), (iii) e (iv) implicam a condição (v) e a condição (vi).

Demonstração: Basta verificar que as equações

$$
\begin{aligned}
& \mathrm{w} \cdot(\mathrm{v} \wedge(\mathrm{x} \wedge \mathrm{u}))=\mathrm{z} \cdot(\mathrm{u} \wedge(\mathrm{y} \wedge \mathrm{v})) \\
& \mathrm{w} \cdot(\mathrm{y} \wedge(\mathrm{x} \wedge \mathrm{u}))=\mathrm{z} \cdot(\mathrm{x} \wedge(\mathrm{y} \wedge \mathrm{v}))
\end{aligned}
$$

são de fato uma identidade.

Vamos chamar de $\mathrm{e}=\mathrm{y} \wedge \mathrm{v}$ e $\mathbf{f}=\mathrm{x} \wedge \mathbf{u}$. Então,

$$
\begin{aligned}
\mathbf{e}= & \left(e_{1}, e_{2}, e_{3}\right)=\left(y_{2} v_{3}-y_{3} v_{2}, y_{3} v_{1}-y_{1} v_{3}, y_{1} v_{2}-y_{2} v_{1}\right) \\
\mathbf{f}= & \left(f_{1}, f_{2}, f_{3}\right)=\left(x_{2} u_{3}-x_{3} u_{2}, x_{3} u_{1}-x_{1} u_{3}, x_{1} u_{2}-x_{2} u_{1}\right) \\
\mathbf{y} \wedge \mathbf{f}= & \left(\left(x_{1} u_{2}-x_{2} u_{1}\right) y_{2}-\left(x_{3} u_{1}-x_{1} u_{3}\right) y_{3},\left(x_{2} u_{3}-x_{3} u_{2}\right) y_{3}\right. \\
& \left.-\left(x_{1} u_{2}-x_{2} u_{1}\right) y_{1},\left(x_{3} u_{1}-x_{1} u_{3}\right) y_{1}-\left(x_{2} u_{3}-x_{3} u_{2}\right) y_{2}\right) \\
\mathbf{w} .(\mathbf{y} \wedge \mathbf{f})= & w_{1}\left(x_{1} u_{2} y_{2}+x_{2} u_{1} y_{1}\right)+w_{1}\left(x_{3} u_{1} y_{3}+x_{1} u_{3} y_{3}\right) \\
& +w_{2}\left(x_{2} u_{3} y_{3}+x_{3} u_{2} y_{3}\right)+w_{2}\left(x_{1} u_{2} y_{1}+x_{2} u_{1} y_{1}\right)+w_{3}\left(x_{3} u_{1} y_{1}+x_{1} u_{3} y_{1}\right) \\
& +w_{3}\left(x_{2} u_{3} y_{2}+x_{3} u_{2} y_{2}\right) \\
= & w_{1} x_{1} u_{2} y_{2}+w_{1} x_{2} u_{1} y_{2}+w_{1} x_{3} u_{1} y_{3}+w_{1} x_{1} u_{3} y_{3}+w_{2} x_{2} u_{3} y_{3} \\
& +w_{2} x_{3} u_{2} y_{3}+w_{2} x_{1} u_{2} y_{1}+w_{2} x_{2} u_{1} y_{1}+w_{3} x_{3} u_{1} y_{1}+w_{3} x_{1} u_{3} y_{1}
\end{aligned}
$$




$$
\begin{aligned}
& +w_{3} x_{2} u_{3} y_{2}+w_{3} x_{3} u_{2} y_{2}+2 w_{1} u_{1} x_{1} y_{1}+2 w_{2} u_{2} x_{2} y_{2}+2 w_{3} u_{3} x_{3} y_{3} \\
= & \left(w_{1} u_{1}\right)(\mathbf{x} \cdot \mathbf{y})+\left(w_{2} u_{2}\right)(\mathbf{x} \cdot \mathbf{y})+\left(w_{3} u_{3}\right)(\mathbf{x} \cdot \mathbf{y})+\left(w_{1} x_{1}\right)(\mathbf{u} \cdot \mathbf{y}) \\
& +\left(x_{2} x_{2}\right)(\mathbf{u} \cdot \mathbf{y})+\left(w_{3} x_{3}\right)(\mathbf{u} \cdot \mathbf{y}) \\
\text { w. }(\mathbf{y} \wedge(\mathbf{x} \wedge \mathbf{u}))= & (\mathbf{w} \cdot \mathbf{u})(\mathbf{x} \cdot \mathbf{y})+(\mathbf{w} \cdot \mathbf{x})(\mathbf{y} \cdot \mathbf{u}) .
\end{aligned}
$$

Calculamos agora $\mathrm{z} \cdot(\mathrm{x} \wedge(\mathrm{y} \wedge \mathrm{v}))$.

$$
\begin{aligned}
& \text { z. }\left(\left(y_{1} v_{2}-y_{2} v_{1}\right) x_{2}-\left(y_{3} v_{1}-y_{1} v_{3}\right) x_{3},\left(y_{2} v_{3}-y_{3} v_{2}\right) x_{3}-\left(y_{1} v_{2}-y_{2} v_{1}\right) x_{1},\right. \\
& \left.\left(y_{3} v_{1}-y_{1} v_{3}\right) x_{1}-\left(y_{2} v_{3}-y_{3} v_{2}\right) x_{2}\right) \\
= & z_{1} x_{2} y_{1} v_{2}+z_{1} v_{2} y_{2} v_{1}+z_{1} x_{3} y_{3} v_{1}+z_{1} x_{3} y_{1} v_{3}+z_{2} x_{3} y_{2} v_{3}+z_{2} x_{3} y_{3} v_{2} \\
& +z_{2} x_{1} y_{1} v_{2}+z_{2} x_{1} y_{2} v_{1}+z_{3} x_{1} y_{3} v_{1}+z_{3} x_{1} y_{1} v_{3}+z_{3} x_{2} y_{2} v_{3}+z_{3} x_{2} y_{3} v_{2} \\
& +2 z_{1} y_{1} x_{1} v_{1}+2 z_{2} y_{2} x_{2} v_{2}+2 z_{3} y_{3} x_{3} v_{3} \\
= & \left(z_{1} y_{1}\right)(\mathbf{x} \cdot \mathbf{v})+\left(z_{2} y_{2}\right)(\mathbf{x} \cdot \mathbf{v})+\left(z_{3} y_{3}\right)(\mathbf{x} \cdot \mathbf{v})+\left(z_{1} v_{1}\right)(\mathbf{x} \cdot \mathbf{y})+\left(z_{2} v_{2}\right)(\mathbf{x} \cdot \mathbf{y})+\left(z_{3} v_{3}\right)(\mathbf{x} \cdot \mathbf{y}) \\
= & (\mathbf{z} \cdot \mathbf{y})(\mathbf{x} \cdot \mathbf{v})+(\mathbf{v} \cdot \mathbf{z})(\mathbf{x} \cdot \mathbf{y}) .
\end{aligned}
$$

Donde podemos escrever a equação

$$
\mathrm{w} \cdot(\mathrm{y} \wedge(\mathrm{x} \wedge \mathrm{u}))=\mathrm{z} \cdot(\mathrm{x} \wedge(\mathrm{y} \wedge \mathrm{v}))
$$

$\mathrm{Na}$ forma

$$
(\mathrm{w} \cdot \mathrm{u})(\mathrm{x} \cdot \mathrm{y})+(\mathrm{w} \cdot \mathrm{x})(\mathrm{y} \cdot \mathrm{u})=(\mathrm{z} \cdot \mathrm{y})(\mathrm{x} \cdot \mathrm{v})+(\mathrm{v} \cdot \mathrm{z})(\mathrm{x} \cdot \mathrm{y})
$$

é de fato uma identidade, pois $\mathbf{w} \cdot \mathbf{u}=\mathbf{v} \cdot \mathbf{z}$ e $\mathbf{x} \cdot \mathbf{w}=\mathbf{z} \cdot \mathbf{y}$ e também $\mathbf{y} \cdot \mathbf{u}=\mathbf{x} \cdot \mathbf{v}$.

Procedendo de maneira análoga com a equação

$$
\mathrm{w} \cdot(\mathrm{v} \wedge(\mathrm{x} \wedge \mathrm{u}))=\mathrm{z} \cdot(\mathrm{u} \wedge(\mathrm{y} \wedge \mathrm{v}))
$$

teremos

$$
\begin{aligned}
& \mathrm{w} \cdot(\mathrm{v} \wedge(\mathrm{x} \wedge \mathrm{u}))=(\mathrm{w} \cdot \mathrm{u})(\mathrm{x} \cdot \mathrm{v})+(\mathrm{w} \cdot \mathrm{x})(\mathrm{u} \cdot \mathrm{v}) \\
& \mathrm{z} \cdot(\mathrm{u} \wedge(\mathrm{y} \wedge \mathrm{v}))=(\mathrm{z} \cdot \mathrm{y})(\mathrm{u} \cdot \mathrm{v})+(\mathrm{v} \cdot \mathrm{z})(\mathrm{u} \cdot \mathrm{v})
\end{aligned}
$$


que é de fato uma identidade, pois $\mathrm{w} \cdot \mathrm{u}=\mathrm{v} \cdot \mathbf{z}$ e $\mathrm{x} \cdot \mathrm{v}=\mathrm{u} \cdot \mathrm{y}$ e também $\mathrm{w} \cdot \mathbf{x}=\mathbf{z} \cdot \mathrm{y}$.

Como conseqüência do lema, segue que dados $\mathrm{x}, \mathrm{y}, \mathrm{u}, \mathrm{v} \in F_{2}^{3}$, para que exista $\gamma$ nas condições requeridas basta que o sistema abaixo tenha solução.

$$
\left\{\begin{array}{l}
x_{1} v_{1}+x_{2} v_{2}+x_{3} v_{3}=y_{1} u_{1}+y_{2} u_{2}+y_{3} u_{3} \\
x_{1} w_{1}+x_{2} w_{2}+x_{3} w_{3}=y_{1} z_{1}+y_{2} z_{2}+y_{3} z_{3} \\
u_{1} w_{1}+u_{2} w_{2}+u_{3} w_{3}=v_{1} z_{1}+v_{2} z_{2}+v_{3} z_{3} \\
e_{1} w_{1}+e_{2} w_{2}+e_{3} w_{3}=f_{1} z_{1}+f_{2} z_{2}+f_{3} z_{3}+\mathrm{y} \cdot \mathbf{z}
\end{array}\right.
$$

onde $e=y \wedge v$ e $f=x \wedge u$.

$\mathrm{O}$ sistema tem solução se o seguinte determinante é não nulo, para cada $\mathrm{z} \in F_{2}^{3}$.

$$
\left|\begin{array}{lll}
x_{1} & x_{2} & x_{3} \\
u_{1} & u_{2} & u_{3} \\
e_{1} & e_{2} & e_{3}
\end{array}\right|,
$$

onde $\mathrm{u}, \mathrm{v} \neq \mathbf{0}$, pois $\mathrm{u} . \mathrm{v}=1$.

$\mathrm{O}$ determinante é nulo se $\{\mathrm{x}, \mathrm{u}, \mathrm{y} \wedge \mathrm{v}\}$ é linearmente dependente ou, equivalentemente, se, e somente se, se verifica uma das seguintes igualdades:

$$
x=0, x=u, x=y \wedge v, y \wedge v=u, y \wedge v=0
$$

Vamos estudar então, separadamente, os casos em que o determinante é zero.

i) $\mathbf{x}=\mathbf{u}$ implica $\mathbf{x} \cdot \mathbf{v}=\mathbf{u} \cdot \mathbf{v}=1=\mathrm{y} \cdot \mathbf{u}$ e também $0=\mathrm{x} \cdot \mathbf{y}=\mathbf{u} \cdot \mathbf{y}=1$. Contradição e, portanto, $\mathrm{x} \neq \mathrm{u}$.

ii) $\mathrm{y} \wedge \mathrm{v}=\mathbf{0}$. Como $\mathrm{v} \neq \mathbf{0}$, podemos ter $\mathrm{y}=\mathbf{0}$ ou $\mathbf{y}=\mathrm{v}$. Se $\mathbf{y}=\mathbf{v}$, então a equaçào $\mathrm{x} \cdot \mathbf{v}=\mathbf{y} \cdot \mathbf{u}$ ficará $\mathrm{x} \cdot \mathrm{v}=\mathbf{v} \cdot \mathbf{u}=1$ e também temos $\mathrm{x} \cdot \mathrm{y}=0$, donde $\mathbf{x} \cdot \mathbf{v}=0$, o que é contradição. Se $y=0$, então o sistema $\left({ }^{*}\right)$ implica em

$$
\left\{\begin{array}{l}
x \cdot w=0 \\
u . w=v \cdot z \\
0=f . z
\end{array}\right.
$$

A equação $f . z=0$ tem soluçōes. Seja $z_{0}$ uma destas soluções não nula, o sistema

$$
\left\{\begin{array}{l}
\mathrm{x} \cdot \mathrm{w}=0 \\
\mathrm{u} \cdot \mathrm{w}=\mathrm{v} \cdot \mathrm{z}_{0}
\end{array}\right.
$$


tem solução, pois $\mathrm{x} \neq \mathrm{u}$, como foi provado em (i). Portanto, nesse caso existe $\gamma$ nas condições requeridas.

iii) $\mathrm{x}=\mathrm{y} \wedge \mathrm{v}$. O sistema $\left({ }^{*}\right)$ implica em

$$
\left\{\begin{array}{l}
\mathrm{x} \cdot \mathrm{w}=\mathrm{y} \cdot \mathrm{z} \\
\mathrm{u} \cdot \mathrm{w}=\mathrm{v} \cdot \mathrm{z} \\
\mathrm{x} \cdot \mathrm{w}=\mathrm{y} \cdot \mathrm{z}+(\mathrm{x} \wedge \mathrm{u}) \mathrm{z}
\end{array}\right.
$$

Como $\mathrm{x} \neq \mathrm{u}$, por (i), a equação $\mathrm{x} \cdot \mathrm{w}=\mathrm{y} \cdot \mathbf{z}+\mathrm{z} \cdot(\mathrm{x} \wedge \mathrm{u})$ tem solução em $\mathbf{z}$. Seja $\mathrm{z}_{0}$ uma destas soluções. Considerando o sistema

$$
\left\{\begin{array}{l}
\mathrm{x} \cdot \mathrm{w}=\mathrm{y} \cdot \mathrm{z}_{0} \\
\mathrm{u} \cdot \mathrm{w}=\mathrm{v} \cdot \mathrm{z}_{0}
\end{array}\right.
$$

encontramos solução para w e, portanto, também neste caso existe $\gamma$ nas condições do enunciado.

iv) $\mathrm{y} \wedge \mathrm{v}=\mathrm{u}$. Já vimos que $\mathrm{x} \neq \mathrm{u}$ e $\mathbf{y} \neq \mathrm{v}$. Neste caso, o sistema $\left({ }^{*}\right)$ resulta

$$
\left\{\begin{array}{l}
\mathrm{x} \cdot \mathrm{w}=\mathrm{y} \cdot \mathrm{z} \\
\mathrm{u} \cdot \mathrm{w}=\mathrm{v} \cdot \mathrm{z} \\
\mathrm{u} \cdot \mathrm{w}=\mathrm{f} \cdot \mathrm{z}+\mathrm{y} \cdot \mathrm{z}
\end{array}\right.
$$

Devemos ter então $v \cdot z=f \cdot z+y \cdot z$ ou ainda $(v-f-y) \cdot z=0$. Esta equação sempre tem soluções. Seja $\mathbf{z}_{0}$ uma soluçào. então temos

$$
\left\{\begin{array}{l}
\mathrm{x} \cdot \mathrm{w}=\mathrm{y} \cdot \mathrm{z}_{0} \\
\mathrm{u} \cdot \mathrm{w}=\mathrm{v} \cdot \mathrm{z}_{0}
\end{array}\right.
$$

Novamente é possível achar soluções para w e também neste caso existe $\gamma$ nas condições requeridas.

v) $\mathbf{x}=0$ implica $\mathrm{x} \wedge \mathrm{u}=0$, donde o sistema $\left({ }^{*}\right)$ ficará com a forma

$$
\left\{\begin{array}{l}
\mathrm{y} \cdot \mathrm{z}=0 \\
\mathrm{v} \cdot \mathrm{z}=\mathrm{u} \cdot \mathrm{w} \\
0=\mathrm{e} \cdot \mathrm{w}
\end{array}\right.
$$

Como $\mathrm{y} \neq \mathrm{v}$ e $\mathrm{y} \neq 0$ (se $\mathrm{y}=0$, então $\alpha=I$ ), $\mathrm{y}, \mathrm{v}$ são linearmente independentes. A equação e.w $=0$ tem solução não nula em w. Seja $w_{0}$ uma destas soluções, então temos

$$
\left\{\begin{array}{l}
\mathrm{y} \cdot \mathrm{z}=0 \\
\mathrm{v} \cdot \mathrm{z}=\mathrm{u} \cdot \mathrm{w}_{0}
\end{array}\right.
$$


Neste caso, é possível achar solução para $\mathrm{z}$ e temos $\gamma$ nas condições requeridas.

(II) Seja $L=\left\langle\alpha, \beta \mid \alpha^{2}=\beta^{3}=(\alpha \beta)^{3}=1\right\rangle$, onde $\alpha=\left[\begin{array}{ll}0 & \mathrm{x} \\ \mathrm{y} & 0\end{array}\right], \beta=\left[\begin{array}{ll}1 & \mathrm{u} \\ \mathrm{v} & 0\end{array}\right]$. Mostraremos que, também nesse caso, é possível encontrar um elemento $\gamma$ como acima.

Os elementos de $L$ agora são os seguintes:

$$
\alpha=\left[\begin{array}{ll}
0 & \mathrm{x} \\
\mathrm{y} & 0
\end{array}\right], \quad \beta=\left[\begin{array}{ll}
1 & \mathrm{u} \\
\mathrm{v} & 0
\end{array}\right], \quad \beta^{2}=\left[\begin{array}{ll}
0 & \mathrm{u} \\
\mathrm{v} & 1
\end{array}\right], \quad \alpha \beta=\left[\begin{array}{cc}
\mathrm{x} \cdot \mathrm{v} & \mathrm{y} \wedge \mathrm{v} \\
\mathrm{y}+\mathrm{x} \wedge \mathrm{u} & \mathrm{y} \cdot \mathrm{u}
\end{array}\right] .
$$

Como $(\alpha \beta)^{3}=1$, temos $\mathbf{x} \cdot \mathbf{v} \neq \mathbf{y} \cdot \mathbf{u}$ ou então $\mathbf{x} \cdot \mathbf{v}=1+\mathbf{y} \cdot \mathbf{u}$.

$$
\begin{aligned}
& (\alpha \beta)^{2}=\left[\begin{array}{cc}
\mathrm{y} \cdot \mathrm{u} & \mathrm{y} \wedge \mathrm{v} \\
\mathrm{y}+\mathrm{x} \wedge \mathrm{u} & \mathrm{x} \cdot \mathrm{v}
\end{array}\right], \quad \alpha \beta^{2}=\left[\begin{array}{cc}
\mathrm{x} \cdot \mathrm{v} & \mathrm{x}+\mathrm{y} \wedge \mathrm{v} \\
\mathrm{x} \wedge \mathrm{u} & \mathrm{y} \cdot \mathrm{u}
\end{array}\right], \\
& \left(\alpha \beta^{2}\right)^{2}=\left[\begin{array}{cc}
\mathrm{y} \cdot \mathrm{u} & \mathrm{x}+\mathrm{y} \wedge \mathrm{v} \\
\mathrm{x} \wedge \mathrm{u} & \mathrm{x} . \mathrm{v}
\end{array}\right] \\
& \alpha \beta \alpha=\left[\begin{array}{cc}
0 & (\mathrm{x} \cdot \mathrm{v}) \mathrm{x}+\mathrm{y} \wedge(\mathrm{x} \wedge \mathrm{u}) \\
(\mathrm{y} \cdot \mathrm{u}) \mathrm{y}+\mathrm{x} \wedge(\mathrm{y} \wedge \mathrm{v}) & 1
\end{array}\right] \\
& (\alpha \beta \alpha)^{2}=\left[\begin{array}{cc}
1 & (\mathrm{x} \cdot \mathrm{v}) \mathrm{x}+\mathrm{y} \wedge(\mathrm{x} \wedge \mathrm{u}) \\
(\mathrm{y} \cdot \mathrm{u}) \mathrm{y}+\mathrm{x} \wedge(\mathrm{y} \wedge \mathrm{v}) & 0
\end{array}\right] \\
& \beta^{2} \alpha \beta=\left[\begin{array}{cc}
\mathrm{y} \cdot \mathrm{u} & (\mathrm{y} \cdot \mathrm{u}) \mathrm{u}+\mathrm{y} \wedge \mathrm{v}+\mathrm{v} \wedge(\mathrm{x} \wedge \mathrm{u}) \\
(\mathrm{x} \cdot \mathrm{v}) \mathrm{v}+\mathrm{y}+\mathrm{x} \wedge \mathrm{u}+\mathrm{u} \wedge(\mathrm{y} \wedge \mathrm{v}) & \mathrm{y} \cdot \mathrm{u}
\end{array}\right] \\
& \beta \alpha \beta^{2}=\left[\begin{array}{cc}
\mathrm{x} \cdot \mathrm{v} & (\mathrm{y} \cdot \mathrm{u}) \mathrm{u}+\mathrm{x}+\mathrm{y} \wedge \mathrm{v}+\mathrm{v} \wedge(\mathrm{x} \wedge \mathrm{u}) \\
(\mathrm{x} \cdot \mathrm{v}) \mathrm{v}+\mathrm{x} \wedge \mathrm{u}+\mathrm{u} \wedge(\mathrm{y} \wedge \mathrm{v}) & \mathrm{x} \cdot \mathrm{v}
\end{array}\right]
\end{aligned}
$$

Mais uma vez, vamos mostrar que é possível escolher $\gamma \in G L L\left(F_{2}\right), \gamma=\left[\begin{array}{cc}0 & \mathrm{z} \\ \mathrm{w} & 0\end{array}\right]$ de tal modo que $(g \gamma)^{2}=1$, para todo $g \in L$, ou seja, basta verificar que os elementos da diagonal principal de $g \gamma$ são iguais para cada $g \in L$.

Se $g=\alpha$, para que $\alpha \gamma=\gamma \alpha$, devemos ter $\mathbf{x} . \mathbf{w}=\mathrm{y} . \mathrm{z}$.

Se $g=\beta$, para que $(\beta \gamma)^{2}=1$, devemos ter u.w $=$ v.z. 
Vamos calcular estes elementos em todos os casos possíveis.

Se $g=\alpha \beta$ ou $g=(\alpha \beta)^{2}$ devemos ter $\mathrm{w} \cdot(\mathrm{y} \wedge \mathrm{v})=\mathrm{yz}+\mathrm{z} \cdot(\mathrm{x} \wedge \mathrm{u})$.

Se $g=\alpha \beta^{2}$ ou $g=\left(\alpha \beta^{2}\right)^{2}$ devemos ter $\mathrm{x} \cdot \mathrm{w}+\mathrm{w} \cdot(\mathrm{y} \wedge \mathrm{v})=\mathrm{z} \cdot(\mathrm{x} \wedge \mathrm{u})$.

Se $g=(\alpha \beta \alpha),(\alpha \beta \alpha)^{2}$ devemos ter

$$
(\mathrm{x} \cdot \mathrm{v})(\mathrm{x} \cdot \mathrm{w})+\mathrm{w} \cdot(\mathrm{y} \wedge(\mathrm{x} \wedge \mathrm{u}))=(\mathrm{y} \cdot \mathrm{u})(\mathrm{y} \cdot \mathrm{z})+\mathrm{z} \cdot(\mathrm{x} \wedge(\mathrm{y} \wedge \mathrm{v}))
$$

Se $g=\beta \alpha \beta^{2}$ devemos ter

$(\mathrm{y} \cdot \mathrm{u})(\mathrm{u} \cdot \mathrm{w})+\mathrm{x} \cdot \mathrm{w}+\mathrm{w} \cdot(\mathrm{y} \wedge \mathrm{v})+\mathrm{w} \cdot(\mathrm{v} \wedge(\mathrm{x} \wedge \mathrm{u}))=(\mathrm{x} \cdot \mathrm{v})(\mathrm{v} \cdot \mathrm{z})+\mathrm{z} \cdot(\mathrm{u} \wedge \mathrm{x})+\mathrm{z} \cdot(\mathrm{u} \wedge(\mathrm{u} \wedge \mathrm{v}))$

Se $g=\beta^{2} \alpha \beta$ devemos ter

$$
(\mathrm{y} \cdot \mathrm{u})(\mathrm{u} \cdot \mathrm{w})+\mathrm{w} \cdot(\mathrm{y} \wedge \mathrm{v})+\mathrm{w} \cdot(\mathrm{v} \wedge(\mathrm{x} \wedge \mathrm{u}))=(\mathrm{x} \cdot \mathrm{v})(\mathrm{v} \cdot \mathrm{z})+\mathrm{z} \cdot(\mathrm{x} \wedge \mathrm{u})+\mathrm{z} \cdot(\mathrm{u} \wedge(\mathrm{y} \wedge \mathrm{v}))
$$

Resumindo, existe $\gamma \in G L L\left(F_{2}\right)$ nas condições requeridas se, e somente se, o sistema abaixo tem solução em $\mathbf{z}$ e w.

(i) $\mathrm{x} \cdot \mathrm{w}=\mathrm{y} \cdot \mathrm{z}$;

(ii) u.w = v.z;

(iii) $\mathrm{w} \cdot(\mathrm{y} \wedge \mathrm{v})=\mathrm{y} \cdot \mathrm{z}+\mathrm{z} \cdot(\mathrm{x} \wedge \mathrm{u})$;

(iv) $\mathrm{x} \cdot \mathrm{w}+\mathrm{w} \cdot(\mathrm{y} \wedge \mathrm{v})=\mathrm{z} \cdot(\mathrm{x} \wedge \mathrm{u})$;

(v) $(\mathrm{x} \cdot \mathrm{v})(\mathrm{x} \cdot \mathrm{w})+\mathrm{w} \cdot(\mathrm{y} \wedge(\mathrm{x} \wedge \mathrm{u}))=(\mathrm{y} \cdot \mathrm{u})(\mathrm{y} \cdot \mathrm{z})+\mathrm{z} \cdot(\mathrm{x} \wedge(\mathrm{y} \wedge \mathrm{v}))$

(vi) $(y \cdot u)(u \cdot w)+w \cdot(y \wedge v)+w \cdot(v \wedge(x \wedge u))=(x \cdot v)(v \cdot z)+z \cdot(x \wedge u)+z \cdot(u \wedge(y \wedge v))$.

A condição (i) implica diretamente que as condições (iii) e (iv) são equivalentes. Como $\mathbf{x} \cdot \mathbf{v}=\mathbf{y} \cdot \mathbf{u}+1$ e pelo Lema 3.5 .5 as condições (i), (ii) e (iv) implicam que

$$
\mathrm{w} \cdot(\mathrm{y} \wedge(\mathrm{x} \wedge \mathrm{u}))=\mathrm{z} \cdot(\mathrm{x} \wedge(\mathrm{y} \wedge \mathrm{v}))
$$

o sistema pode ser escrito como 
(i) $\mathrm{x} \cdot \mathrm{w}=\mathrm{y} \cdot \mathrm{z}$;

(ii) $\mathrm{u} . \mathrm{w}=\mathrm{v} \cdot \mathrm{z}$;

(iii) $\mathrm{w} \cdot(\mathrm{y} \wedge \mathrm{v})=\mathrm{x} \cdot \mathrm{w}+\mathrm{z} \cdot(\mathrm{x} \wedge \mathrm{u})$;

ou ainda

$$
\left\{\begin{array}{l}
\mathrm{x} \cdot \mathrm{w}=\mathrm{y} \cdot \mathrm{z} \\
\mathrm{u} \cdot \mathrm{w}=\mathrm{v} \cdot \mathrm{z} \\
0=\mathrm{y} \cdot \mathrm{z} \\
\mathrm{w} \cdot(\mathrm{y} \wedge \mathrm{v})=\mathrm{x} \cdot \mathrm{w}+\mathrm{z} \cdot(\mathrm{x} \wedge \mathrm{u})
\end{array}\right.
$$

Dados $\mathrm{x}, \mathrm{y}, \mathrm{u}, \mathrm{v} \in F_{2}^{3}$, para que exista $\gamma$ nas condições requeridas, basta que o sistema abaixo tenha solução.

$$
\left\{\begin{array}{l}
\mathrm{x} \cdot \mathrm{v}=1+\mathrm{y} \cdot \mathrm{u} \\
\mathrm{x} \cdot \mathrm{w}=0 \\
\mathrm{u} \cdot \mathrm{w}=\mathrm{v} \cdot \mathrm{z} \\
\mathrm{e} \cdot \mathrm{w}=\mathrm{f} \cdot \mathrm{z}
\end{array}\right.
$$

onde $e=(y \wedge v)$ e $f=(x \wedge u)$.

$\mathrm{O}$ sistema tem solução se o seguinte determinante é não nulo para cada $\mathbf{z} \in F_{2}^{3}$.

$$
\left|\begin{array}{lll}
x_{1} & x_{2} & x_{3} \\
u_{1} & u_{2} & u_{3} \\
e_{1} & e_{2} & e_{3}
\end{array}\right|,
$$

onde $\mathrm{x}, \mathrm{y}, \mathrm{u}, \mathrm{v} \neq 0$.

$\mathrm{O}$ determinante é nulo se $\{\mathrm{x}, \mathrm{u}, \mathrm{y} \wedge \mathrm{v}\}$ é linearmente dependente ou, equivalentemente, se, e somente se, se verificam as identidades

$$
\mathrm{x}=\mathrm{u}, \quad \mathrm{x}=\mathrm{y} \wedge \mathrm{v}, \quad \mathrm{y} \wedge \mathrm{v}=0 \quad \text { ou } \quad \mathrm{y} \wedge \mathrm{v}=\mathrm{u}
$$

Vamos estudar separadamente cada caso.

i) $\mathrm{x}=\mathrm{y} \wedge \mathrm{v}$ implica $\mathrm{x} \cdot \mathrm{v}=(\mathrm{y} \wedge \mathrm{v}) \cdot \mathrm{v}=0=1+\mathrm{y} \cdot \mathrm{u}$. Mas $\mathrm{x} \cdot \mathrm{y}=1=(\mathrm{y} \wedge \mathrm{v}) \cdot \mathrm{y}=0$, o que é uma contradiçào. Portanto, $\mathrm{x} \neq \mathrm{y} \wedge \mathrm{v}$.

ii) $\mathrm{y} \wedge \mathrm{v}=\mathbf{0}$ implica $\mathrm{y}=\mathrm{v}$. Temos então $\mathrm{x} \cdot \mathrm{v}=1+\mathrm{y} \cdot \mathrm{u}=1+\mathrm{u} \cdot \mathrm{v}=0$. Por outro lado, $\mathrm{x} \cdot \mathrm{y}=1=\mathrm{x} \cdot \mathrm{v}$, o que é contradição. 
iii) $\mathrm{x}=\mathrm{u}$ implica $\mathrm{x} \cdot \mathrm{v}=\mathrm{u} \cdot \mathrm{v}=1=1+\mathrm{y} \cdot \mathrm{u}$, donde $\mathrm{y} \cdot \mathrm{u}=0$. Por outro lado, $\mathrm{x} \cdot \mathrm{y}=1=$ u.y, o que é contradição.

iv) $\mathrm{y} \wedge \mathrm{v}=\mathrm{u}$. Temos então

$$
\left\{\begin{array}{l}
\mathrm{x} \cdot \mathrm{w}=0 \\
\mathrm{u} \cdot \mathrm{w}=\mathrm{v} \cdot \mathrm{z} \\
\mathrm{u} \cdot \mathrm{w}=\mathrm{f} \cdot \mathrm{z}
\end{array}\right.
$$

o que implica $v \cdot z=f . z$, ou seja, $(v-\mathbf{f}) \cdot z=0$. Esta equação sempre tem soluções.

Seja $z_{0}$ uma solução, então temos

$$
\left\{\begin{array}{l}
\mathrm{x} \cdot \mathrm{w}=0 \\
\mathrm{u} \cdot \mathrm{w}=\mathrm{v} \cdot \mathrm{z}_{0}
\end{array}\right.
$$

Como $\mathrm{x}, \mathrm{u}$ são linearmente independentes, então o sistema tem solução e é possível encontrar $\gamma$ nas condições requeridas.

Agora usaremos um argumento semelhante ao utilizado por Chein na demonstração do Teorema 1 [05] para mostrar que $\langle L, \gamma\rangle \cong M_{24}$.

Com efeito, como vimos até agora, em ambos os casos possiveis podemos escolher $\gamma \in G L L\left(F_{2}\right)$, tal que $g \gamma$ é de ordem 2, para todo $g \in L$. Assim,

$$
(g \gamma)^{2}=(g \gamma)(g \gamma)=1
$$

donde $g \gamma=\gamma g^{-1}$.

Pela diassociatividade e usando a identidade $(a b)(c a)=[a(b c)] a$, temos que

$$
g_{1}\left(\gamma^{n} g_{2}\right)=\left(g_{2} g_{2}^{-1} g_{1}\right)\left(\gamma^{n} g_{2}\right)=g_{2}\left[\left(g_{2}^{-1} g_{1}\right) \gamma^{n}\right] g_{2}
$$

Se $n=0$. então o produto acima é apenas $g_{1} g_{2}$ e. portanto. está em $L$.

Se $n=1$, então $g_{2}\left[\left(g_{2}^{-1} g_{1}\right) \gamma\right]$ pode ter ordem 2 oủ 3 .

Já vimos que $\left(g_{2}^{-1} g_{1}\right) \gamma$ tem ordem $2, \forall g_{1}, g_{2} \in L$. Provaremos o seguinte

Lema 3.5.6 Nas condições acima, temos $g_{1}\left(\gamma g_{2}\right) \in L$ ou $g_{1}\left(\gamma g_{2}\right) \in L \gamma$. 
Demonstração: Se $g_{2}\left[\left(g_{2}^{-1} g_{1}\right) \gamma\right]$ tem ordem 2, então temos

$$
g_{1}\left(\gamma g_{2}\right)=(g_{2} \underbrace{\left[\left(g_{2}^{-1} g_{1}\right) \gamma\right]}_{\text {tem ordem } 2}) g_{2}=\left(\left[\left(g_{2}^{-1} g_{1}\right) \gamma\right]^{-1} g_{2}^{-1}\right) g_{2} .
$$

Pela diassociatividade,

$$
\left(\left[\left(g_{2}^{-1} g_{1}\right) \gamma\right]^{-1} g_{2}^{-1}\right) g_{2}=\left[\left(g_{2}^{-1} g_{1} \gamma\right]^{-1} g_{2}^{-1} g_{2}=\left[\left(g_{2}^{-1} g_{1}\right) \gamma\right]^{-1}=\left(g_{2}^{-1} g_{1}\right) \in L_{\gamma}\right.
$$

Se $g_{2}\left[\left(g_{2}^{-1} g_{1}\right) \gamma\right]$ tem ordem 3 , então temos

$$
g_{2}\left[\left(g_{2}^{-1} g_{1}\right) \gamma\right]=\left[\left(g_{2}^{-1} g_{1}\right) \hat{\imath}_{i}\right]^{-2} g_{2}^{-2}=\left(\left[\left(g_{2}^{-1} g_{1}\right) \gamma\right]^{2}\right)^{-1} g_{2}^{-2}=g_{2}^{-2} \text {. }
$$

Logo,

$$
g_{1}\left(\gamma g_{2}\right)=g_{2}\left[\left(g_{2}^{-1} g_{1}\right) \gamma\right] g_{2}=g_{2}^{-2} \cdot g_{2}=g_{2}^{-1} \in L
$$

Teorema 3.5.7 O conjunto $L \cup L \gamma$ é fechado para a multiplicação, logo é um subloop isomorfo a $\mathrm{M}_{24}$.

Demonstração: De fato, sejam $g_{1}, g_{2} \in L$ e $m, n=0,1$. Calculemos

$$
\begin{aligned}
\left(g_{1} \gamma^{m}\right)\left(g_{2} \gamma^{n}\right)= & \left\{\left[\left(g_{1} \gamma^{m}\right)\left(g_{2} \gamma^{n}\right)\right] \gamma^{m}\right\} \gamma^{-m} \\
& \text { (usando uma das identidades de Moufang) } \\
= & \left\{g_{1}\left[\gamma^{m}\left(g_{2} \gamma^{n+m}\right)\right]\right\} \gamma^{-m}=A .
\end{aligned}
$$

Se $m=1$ e $n=0$, então

$$
A=\left\{g_{1}\left[\gamma\left(g_{2} \gamma\right)\right]\right\} \gamma=\left\{g_{1}\left[\gamma \gamma g_{2}^{-1}\right]\right\} \gamma=\left(g_{1} g_{2}^{-1}\right) \gamma \in L \gamma
$$

Se $m=0$ e $n=1$, então

$$
A=\left[g_{1}\left(g_{2} \gamma\right)\right]=g_{1}\left(\gamma g_{2}^{-1}\right)
$$

Pelo Lema 3.5.6. $g_{1}\left(\gamma g_{2}^{-1}\right)=g_{2}^{-1}$ ou $g_{3} \gamma$, donde $g_{1}\left(\gamma g_{2}^{-1}\right)$ está em $L$ ou em $L \gamma$. 
Se $m=1$ e $n=1$, então

$$
A=\left\{g_{1}\left[\gamma g_{2}\right]\right\} \gamma
$$

Novamente, pelo Lema 3.5.6, $g_{1}\left(\gamma g_{2}\right)=g_{2}^{-1}$ ou $g_{3} \gamma$, donde $g_{1}\left(\gamma g_{2}\right) \gamma=\left\{\begin{array}{l}g_{2}^{-1} \gamma \\ g_{3}\end{array}\right.$

Ou seja, $g_{1}\left(\gamma g_{2}\right) \gamma$ está em $L$ ou $L \gamma$.

\section{Subloops de ordem 32}

Suponha que exista $H$ um subloop de $G L L\left(F_{2}\right)$ de ordem 32 .

Como $|H|=32$, então todo elemento tem ordem 2 e pela Proposição 2 [05, p.35], $H$ é um grupo abeliano. Logo, $H$ é isomorfo a $C_{2} \times C_{2} \times C_{2} \times C_{2} \times C_{2}$, o que é uma contradição, pois em $G L L\left(F_{2}\right)$ não há grupos isomorfos a $C_{2} \times C_{2} \times C_{2} \times C_{2}$.

\section{Subloops de ordem 48}

Suponha que exista $H$ subloop de $G L L\left(F_{2}\right)$ tal que $|H|=48$. Então, $H$ deveria ter um 2-subgrupo de Sylow de ordem 16 e já vimos que em $G L L\left(F_{2}\right)$ não tem subgrupo de ordem 16.

Em função dos resultados deste capítulo, podemos enunciar o seguinte

Teorema 3.5.8 Um subloop $L$ de $G L L\left(F_{2}\right)$ é maximal se, e somente se, ele é isomorfo a $C_{2} \times C_{2} \times C_{2}, M_{12}$ ou $M_{24}$. 


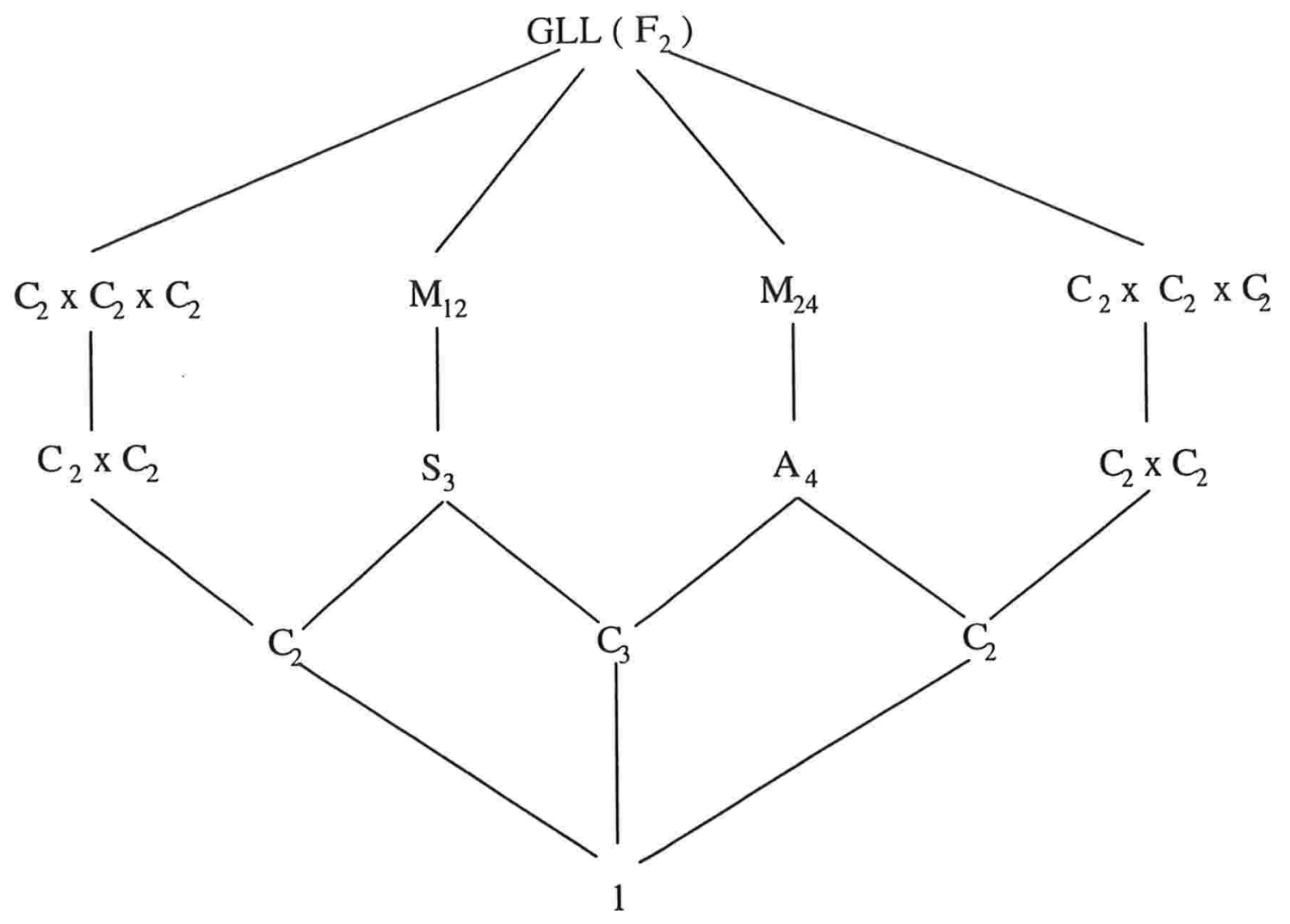

Figura 3.1:

Diagrama parcial do reticulado de subloops de $G L L\left(F_{2}\right)$ mostrando as relações de inclusão, a menos de isomorfismos. 


\section{Referências bibliográficas}

[01] ARTIN, E. Geometric algebra. Interscience, New York, 1964.

[02] BAUMSLAG, F.L. Theory and problems of group theory. McGraw Hill, New York, 1968. (Shaum's Outline Series)

[03] BRUCK, R.H. A survey of binary systems. Springer, Berlin, 1958.

[04] CHEIN, O. Moufang loops of small order. Memoirs of the American Mathematical Society, 197, 13 (1978).

[05] CHEIN, O. Moufang loops of small order I. Trans. Am. Math. Soc., v.188, (1974), 31-51.

[06] CheiN, O.; Pflugfelder, H.O. The smallest Moufang loop. Arch. Math., v.22, (1971), 573-576.

[07] GOOD.AIRE, E.G.; JESPERS, E.; POLCINO MILIES, F.C. Alternative loop rings. North Holland Math. Studies, 184, Elsevier, Amsterdam, 1996.

[0S] KARG.APOLOV, M.I.; MERZLJAKOV, J.I. Fundamentals of the theory of groups. 2.ed.rev. Springer, New York, 1979. (Graduate Texts in Mathematics, 62)

[09] LIEBECK, M.W. The classification of finite simple Moufang loops. Math. Proc. Camb. Phil. Soc., 102 (1987), 33-47.

[10] PAIGE. L.J. A class of simple Moufang loops. Proc. Amer. Math. Soc., 7 (1956), 471-482.

[11] PFLLGFELDER, H.O. Quasigroups and loops: introduction. Heldermann, Berlin, 1990. (Sigma Series in Pure Mathematics, 7)

[12] SCHAFER. R.D. An introduction to nonassociative algebras. Dover, New York, 1994. 
[13] SCOTT, W.R. Group theory. Prentice-Hall, Englewood Cliffs, 1974.

[14] SUPRUnenkiO, D.A. Matrix groups. Am. Math. Soc., Providence, Rhode Island, 1976.

[15] THOMAS, A.D.; WOOD, G.V. Group tables. Shiva, Orpington, 1980.

[16] ZHEVLAKOV, K.A.; SHESTAKOV, I.P.; SHIRSKOV, A.I.; SLIN'KO, A.M. Rings that are nearly associative. Academic Press, New York, 1982. (Pure and Applied Mathematics, 104) 